TMS-EEG:

First steps towards a clinical application in epilepsy

Esther ter Braack 



\section{Propositions}

Accompanying the thesis

\section{TMS-EEG: first steps towards a clinical application in epilepsy}

1) A parameter that is robust only when using a very strict measuring protocol, cannot be used in clinical practice

2) Complete understanding of the TEP is not necessary for clinical utility

3) A silent TMS coil is required for optimal N100/P180 evaluation

4) Defining TMS as a non-invasive technique is inexplicable

5) The search for significant differences has nothing to do with clinically relevant findings

6) Progress would be faster if scientists would share more with each other

7) Technology develops faster than our ability to adequately use it

8) There is an important task for the Technical Physician in the medical device industry

9) Life is what happens to us, while we are making other plans (Allen Saunders)

Esther ter Braack

6 June 2018 


\section{Stellingen}

Behorende bij het proefschrift

\section{TMS-EEG: first steps towards \\ a clinical application in epilepsy}

1) Een meetrespons die alleen robuust is met een zeer sterk gecontroleerd meetprotocol, is niet bruikbaar in de klinische praktijk

2) Volledig begrip van de TEP is niet vereist voor klinisch gebruik

3) Een stille TMS spoel is nodig voor optimale N100/P180 evaluatie

4) TMS definiëren als een niet-invasieve techniek is niet uit te leggen

5) De zoektocht naar significante verschillen heeft niets te maken met klinisch relevante resultaten

6) De vooruitgang zou sneller gaan als wetenschappers meer met elkaar zouden delen

7) Technologie ontwikkelt zich sneller dan ons vermogen om deze adequaat te gebruiken

8) Er ligt een belangrijke taak voor de Technisch Geneeskundige in de medische technologie industrie

9) Het leven is wat ons overkomt, terwijl we andere plannen maken (Allen Saunders)

Esther ter Braack

6 juni 2018 


\section{TMS-EEG: FIRST STEPS TOWARDS A CLINICAL APPLICATION IN EPILEPSY}

ESTHER M. TER BRAACK 


\section{Samenstelling promotiecommissie:}

Voorzitter/Secretaris

Prof. dr. ir. J.W.M. Hilgenkamp Universiteit Twente

\section{Promotor}

Prof. dr. ir. M.J.A.M. van Putten

Universiteit Twente

\section{Leden}

Prof. dr. K. Vonck

Prof. dr. ir. D.F. Stegeman

Dr. H.J. Schelhaas

Prof. dr. S.A. van Gils

dr. E.H.F. van Asseldonk

Universiteit Gent

Vrije Universiteit Amsterdam

Kempenhaeghe

Universiteit Twente

Universiteit Twente

\section{Paranimfen:}

Marleen Tjepkema-Cloostermans

Annika de Goede

Cover design: Guus Rolsma

Printed by Ipskamp Printing

The research described in this thesis was performed at the department of Clinical Neurophysiology at the University of Twente, Enschede and at the department of Clinical Neurophysiology at the Medisch Spectrum Twente, Enschede.

This research was funded by the Dutch PIDON grant, supported by the Dutch Ministry of Economic Affairs and the Province of Overijssel.

TMS-EEG: First steps towards a clinical application in epilepsy

Ph.D. Thesis, University of Twente

ISBN 978-94-028-1072-1

Copyright $\odot 2018$ by E.M. ter Braack, Enschede, the Netherlands 


\title{
TMS-EEG: FIRST STEPS TOWARDS A CLINICAL APPLICATION IN EPILEPSY
}

\section{PROEFSCHRIFT}

\author{
ter verkrijging van \\ de graad van doctor aan de Universiteit Twente, \\ op gezag van de rector magnificus, \\ prof. dr. T.T.M. Palstra, \\ volgens besluit van het College voor Promoties \\ in het openbaar te verdedigen \\ op woensdag 6 juni 2018 om 12:45 uur
}

door

Esther Maria ter Braack

geboren op 4 juni 1984

te Enschede 
Dit proefschrift is goedgekeurd door de promotor:

prof. dr. ir. M.J.A.M. van Putten 


\section{Table of contents}

1. General introduction

2. Reduction of TMS induced artifacts in EEG using Principal Component Analysis

3. Masking the Auditory Evoked Potential in TMS-EEG: a comparison of various methods

4. Resting motor threshold and TMS-EMG-EEG evoked responses during daytime

5. Early TMS evoked potentials in epilepsy: a pilot study

6. General discussion

Summary

Samenvatting

Dankwoord

About the author 



\section{Chapter 1}

\section{General introduction}

Partly based on:

Annika A. De Goede, Esther M. ter Braack, Michel J.A.M. van Putten 'Single and paired pulse transcranial magnetic stimulation in drug naïve epilepsy.' Clinical Neurophysiology 2016;127:3140-3155 


\section{Epilepsy}

The human brain consists of billions of neurons, which form dedicated neuronal networks. Normal physiological brain function depends partly on a balance between excitation and inhibition of these different neuronal networks, where the timing and level of activation is critical. This complex process is realized through various types of neurons, synapses and neurotransmitters, all having specific excitatory or inhibitory properties. The balance between excitation and inhibition is delicate, and when it is disturbed abnormal brain functioning may arise.

Epilepsy, resulting from such an imbalance in excitation and inhibition (McCormick and Contreras 2001), is characterized by the occurrence of seizures during which there is a pathologically increased synchronization between neuronal networks. Seizures can be categorized as focal, secondary generalized or primary generalized. Within the overall classification of focal or generalized epilepsy, different subtypes of epilepsy can be distinguished. The prevalence of epilepsy is estimated at 5.8 per 1000 persons in developed countries, 10.3 per 1000 persons for urban and 15.8 per 1000 persons for rural areas in developing countries (Ngugi et al. 2010). Approximately $10 \%$ of the population experiences one or more seizures during their lifetime when single unprovoked seizures, acute symptomatic seizures (after trauma, stroke, infection etc.), febrile seizures and epilepsy are all included (Annegers et al. 1995). As seizures are disturbing events, typically associated with mental and physical discomfort, preventing seizures is one of the key priorities in epilepsy management. It has recently been shown that vasoconstriction and hypoxia occur after a seizure, which can lead to postictal sensory, cognitive and motor impairments (Farrell et al. 2016). Further, frequent or prolonged seizures in the developing and maturing brain can result in long-lasting detrimental effects, including epileptogenesis (Ben-Ari and Holmes 2006). In addition, although a single seizure may not have damaging effects on the brain, there is evidence that recurring seizures result in a decline in cognitive function (Hermann et al. 2006).

A patient is diagnosed with epilepsy when 1) there have been two unprovoked seizures with an interval of $>24$ hours; or when 2) there has been one seizure but there is an increased risk of more seizures; or when 3) the patient's history already indicates a specific epilepsy syndrome (Fisher et al. 2014). An increased risk of more seizures exists if an interictal electroencephalogram (EEG) shows epileptiform discharges, or if structural brain abnormalities are seen on a magnetic resonance 
imaging (MRI) scan. In patients with a clear history of two or more seizures, or EEG or MRI abnormalities, criterion 1, 2 or 3 applies, and in most of these patients treatment to prevent further seizures is started.

In the majority of newly diagnosed epilepsy patients an anti-epileptic drug (AED) of first choice is prescribed. The patient ideally becomes seizure-free on monotherapy, but when a single AED is not effective, a combination of two or more AEDs can be used. Approximately 20-30\% of epilepsy patients do not become seizure free on AEDs and suffer from pharmacoresistant or refractory epilepsy (Kwan and Sander 2004; Picot et al. 2008). They may spend many months or years trying different (combinations of) AEDs with their accompanying side effects. Other treatment options are then typically explored, such as a ketogenic diet, epilepsy surgery or vagus nerve stimulation. It is important to identify pharmacoresistance as early as possible to prevent a decline in cognitive and developmental function (Berg 2009). In all patients, evaluation of AED success is based on the absence or recurrence of the seizures, so during this trial and error based process additional seizures may occur. It can take up to several months to either achieve an effective dosage and/or combination of AEDs, meaning that the patient becomes seizure free; or conclude that the patient suffers from refractory epilepsy. Hence, a major challenge is to shorten the time needed to evaluate the success of AEDs on a single subject level.

In patients with only one seizure, and a normal EEG and MRI, uncertainty remains. A seizure is a traumatic experience, and an epilepsy diagnosis has potential serious consequences, such as the loss of a driving licence or the need for career changes. Currently there is only one option in these patients: wait if a second seizure occurs. The estimated probability of seizure recurrence in patients with a normal EEG is 27.4\% (Krumholz et al. 2007). This means that more than a quarter of these patients do have epilepsy, but the definite diagnosis is made only at a later stage. Therefore, another major challenge is to improve the diagnostic process in patients who present with a single seizure and a normal EEG and MRI scan.

When we consider these two major challenges, namely improving diagnostics and shortening therapy evaluation, there is a need for a new investigational tool for both first seizure patients and newly diagnosed epilepsy patients who start taking AEDs. These challenges are part of the research priorities defined by the US National 
Institute of Neurological Disorders and Stroke in $2014^{1}$. When we do not want to use seizure recurrence as guidance, we need to assess whether there (still) is an increased risk of seizures in first seizure patients or patients taking AEDs. The EEG seems to be a good candidate: more and longer EEG recordings may reveal more interictal events which can give more insight in the disease status (Geut et al. 2017). Another option is to assess the (dis)balance between excitation and inhibition, using a technique of which its potential use in epilepsy has only recently been recognized: Transcranial Magnetic Stimulation (TMS).

\section{Transcranial magnetic stimulation (TMS)}

TMS is based on the fundamental principles of electromagnetic induction. The TMS coil is placed directly on the head of the subject, and the pulse of electric current in the coil produces a magnetic field which is oriented perpendicular to the coil. This time-varying magnetic field induces electrical currents in the cortex which, if large enough, depolarize neurons and initiate action potentials (Barker et al. 1985; Hallett 2000; Rothwell 1997). As the magnetic field attenuates rapidly with increasing distance from the coil, stimulation is focal and limited to the superficial cortical layers. TMS is a technique with several possible readouts to measure excitability (Figure 1.1), where we define excitability as the strength of the response of cortical neurons to an external stimulus.

Stimulation can be applied using single pulses (spTMS), two paired pulses with a variable interstimulus interval (ppTMS) or trains of pulses at a specific frequency (repetitive TMS: rTMS). Single and paired pulse TMS are used to assess (changes in) excitability, while with rTMS changes in excitability can be induced. Although in theory any superficial cortical brain area can be stimulated by TMS, the most commonly used stimulation site is the primary motor cortex, due to its characteristic signature output: the motor evoked potential (MEP).

\footnotetext{
${ }_{1}$ https://www.ninds.nih.gov/About-NINDS/Strategic-Plans-Evaluations/Strategic-Plans/2014-NINDSBenchmarks-Epilepsy-Research
} 


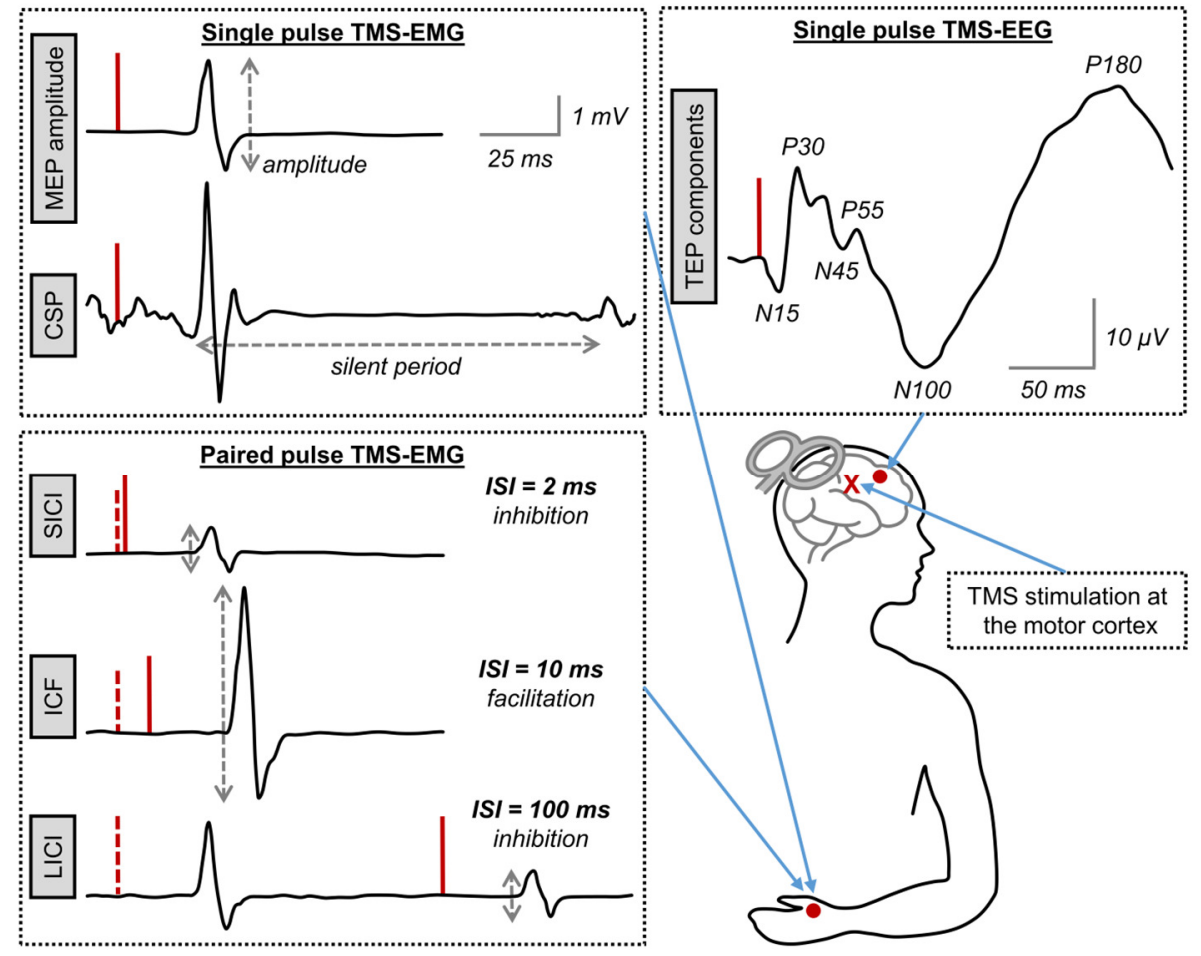

Figure 1.1. Outcome measures for TMS-EMG and TMS-EEG

Upper panels correspond to the single pulse TMS paradigm and the lower panel to the paired pulse TMS paradigm. Red straight lines $=$ TMS pulse, red dashed lines $=$ conditioning TMS pulse. $\mathrm{MEP}=$ motor evoked potential, $\mathrm{CSP}=$ cortical silent period, TEP $=$ TMS evoked potential, $\mathrm{SICI}=$ short intracortical inhibition, $\mathrm{ICF}=$ intracortical facilitation, $\mathrm{LICI}=$ long intracortical inhibition and ISI $=$ interstimulus interval. 


\section{TMS combined with EMG}

For spTMS traditional measures are the resting motor threshold, the MEP amplitude, and the cortical silent period (CSP). The resting motor threshold (RMT or MT) is defined as the minimum TMS intensity needed to elicit at least five out of ten MEPs of at least $50 \mu \mathrm{V}$ in a relaxed target muscle (Rossini et al. 1994). The MEP amplitude is the peak-to-peak amplitude of the response measured in the target muscle after a TMS pulse. When the target muscle is (slightly) activated, a TMS pulse results in an interruption of this voluntary muscle activity, and the duration of the interruption is called the CSP.

With the ppTMS paradigm there are three measures available, based on three different interstimulus intervals (ISI). The first TMS pulse is a conditioning pulse and the MEP after the second TMS pulse is the conditioned MEP. With an ISI of 15 ms short intracortical inhibition (SICI) occurs, which means that the amplitude of the conditioned MEP is smaller than the amplitude of the unconditioned MEP. SICI is thought to be related to GABA-A mediated inhibition (Hanajima et al. 1998; Kujirai et al. 1993). With an ISI of 6-30 ms the conditioned MEP is larger in amplitude than the unconditioned MEP; this is known as intracortical facilitation (ICF). Facilitation is the net result of N-methyl-D-aspartate (NMDA) mediated facilitation and GABA-A mediated inhibition (Hanajima et al. 1998; Inghilleri et al. 1996; Schwenkreis et al. 1999; Ziemann et al. 1998). Finally, with an ISI of 50-400 $\mathrm{ms}$, long intracortical inhibition (LICI) is measured, with the conditioned MEP again smaller in amplitude than the unconditioned MEP. LICI is related to GABA-B mediated inhibition (McDonnell et al. 2006; Pierantozzi et al. 2004; Werhahn et al. 1999). SICI, ICF and LICI are calculated as a ratio between the amplitudes of the conditioned MEP and an unconditioned MEP, and this ratio can be compared within a subject or between subjects. 


\section{TMS combined with EEG}

The effect of a TMS pulse applied to the cortex can also be assessed by measuring the neuronal response in the stimulated brain using EEG (Ilmoniemi and Kičić 2010; Ilmoniemi et al. 1997). TMS-EEG offers a more direct measure of cortical excitability than measures based on TMS-EMG, as it is not influenced by the excitability of corticospinal and spinal neurons. Another advantage of TMS-EEG is that is also possible to stimulate and measure responses in other brain areas than the primary motor cortex. The average EEG response obtained after averaging over multiple single TMS pulses is called the TMS evoked potential (TEP). The largest TEP amplitudes are measured directly under the TMS coil and diminish with increasing distance from the stimulated brain area (Ilmoniemi and Kičić 2010; Komssi et al. 2002). The TEP has distinct characteristics (see Figure 1). Negative components at 15, 45, and $100 \mathrm{~ms}$ and positive components at 30,60, and $180 \mathrm{~ms}$ have been reported in several studies (Casarotto et al. 2010; Ilmoniemi and Kičić 2010; Komssi et al. 2004), with some authors describing even earlier components at 7-10 ms and 13-14 ms (Bonato et al. 2006; Ferreri et al. 2011). Using a protocol with specific GABA-ergic drugs in healthy subjects, it has been shown that the N45 is related to GABA-A receptor mediated inhibition, whereas the N100 is linked to GABA-B receptor mediated inhibition (Premoli et al. 2014).

TMS-EEG is technically challenging, because the intensity of the TMS pulse is very high (1-2 tesla) and stimulation is, although targeted at a pre-defined cortical area, not very specific. First of all, dedicated measuring equipment is necessary (Ilmoniemi and Kičić 2010). To avoid saturation of the amplifier a sample-and-hold amplifier or a DC amplifier should be used. In addition, the EEG electrodes need to be TMS compatible, since standard electrodes can heat up with the TMS pulse. After collecting the TMS-EEG signals, various artefacts can hamper the interpretation of the response. The TMS pulse itself induces a large artefact, with amplitudes in the order of $\mathrm{mV}$ and duration of $<5 \mathrm{~ms}$. The TMS pulse can also induce scalp muscle activity, leading to a second large bipolar artefact which decays slowly and can last up to $40-50 \mathrm{~ms}$. A third artefact is the auditory evoked potential (AEP), as each TMS pulse is accompanied by a clicking sound. The AEP consists of components which overly the TEP, with amplitudes that increase with higher TMS stimulation intensities. 


\section{TMS in epilepsy}

Several studies have been published investigating the difference in excitability between epilepsy patients and healthy subjects. It is important to point out that AEDs most likely influence excitability (Ziemann et al. 2015). A systematic overview of all TMS-EMG studies in drug naive epilepsy patients can be found in a recent review (de Goede et al. 2016). Indeed, differences in excitability measures were found in epilepsy patients after starting AEDs. When we look at drug naive epilepsy patients, spTMS suggests increased cortical excitability along with enhanced inhibitory mechanisms for generalized epilepsy, as reflected by a trend towards a decreased RMT and a prolonged CSP. However, inconclusive findings for RMT, MEP amplitude and CSP indicate the limited applicability of single pulse TMS outcomes in focal epilepsy patients. ppTMS (SICI and LICI) shows the most consistent findings of increased excitability for both focal and generalized epilepsy. Therefore, when the MEP is used as TMS readout, ppTMS seems to be more sensitive to detect changes in cortical excitability than spTMS. Although the findings of ppTMS studies are the most consistent and promising, it should be noted that the majority of positive studies were from a single research group. Even though they have confirmed their own findings multiple times for different types of epilepsy, these results have not been reproduced by other authors (Bauer et al. 2018).

For TMS-EEG, no studies in drug naive epilepsy patients have been published yet, but there are a few reports on epilepsy patients using AEDs. Differences in the TEP were found between healthy subjects and patients with Unverricht-Lundborg type progressive myoclonus epilepsy (EPM1) and juvenile myoclonic epilepsy (JME) (Del Felice et al. 2011; Julkunen et al. 2013). Although both studies show differences in the N100 component between patients and controls, the N100 was decreased in EPM1 patients and increased in JME patients. A recent study failed to find significant differences in the TEP between genetic generalized epilepsy patients and healthy controls (Kimiskidis et al. 2017). When stimulating other sites than the motor cortex, focal epilepsy patients showed an increase in late activity (300 to $1000 \mathrm{~ms}$ after TMS) (Shafi et al. 2015; Valentin et al. 2008). These promising results warrant further investigations of TMS-EEG in epilepsy patients. 


\section{Goals}

TMS is used extensively in neuroscience, but clinical applications are limited. This thesis explores if it is feasible to use TMS-EEG for the diagnostic process and evaluation of treatment response in epilepsy patients. We focus on analysis of TEP responses after stimulating the motor cortex with spTMS using a robot-navigated TMS-EEG set-up.

The first goal is to investigate two methods to reduce artifacts from the TEP. As discussed before, there are at least three recognizable artifacts: the TMS pulse artifact, the long-lasting decay muscle artifact, and the auditory evoked potential. The latter two artifacts are overlying the TEP components, which makes analysis without artifact rejection difficult or even impossible.

The second goal is to study the influence of the time of day on the TEP. For TMSEEG to be of use in a clinical setting, the variation due to changes in measurement parameters should be small in comparison to the difference in response between healthy subjects and epilepsy patients. In addition, if a very strict measurement protocol is needed, TMS-EEG may be too difficult to implement in daily practice.

The third and last goal of this thesis is to investigate the differences in the TEP between healthy subjects and epilepsy patients. After the promising results from ppTMS we explored whether also spTMS-EEG, as an even more direct measurement of excitability, has potential clinical use in epilepsy diagnostics.

\section{Outline of this thesis}

In chapter 2 we present a method to reduce TMS-induced magnetic and muscle artifacts in the EEG, using principal component analysis (PCA). We apply PCA on data measured in healthy subjects after stimulating the left and right motor cortex and evaluate the effects of removing principal components on the amplitude of the artifact and TEP. In chapter 3 we investigate different ways to minimize the auditory artifact in the response. In chapter 4 we explore the daytime variation in the TEP. We performed five TMS-EEG sessions between 8AM and 6PM in a group of healthy subjects. Finally, chapter 5 deals with a first step towards clinical application of TMS-EEG. We compare the TEP of epilepsy patients with healthy controls. 


\section{References}

Annegers JF, Hauser WA, Lee JR-J, Rocca WA. "Incidence of acute symptomatic seizures in Rochester, Minnesota 1935-1984". Epilepsia 1995; 36: 327-333

Barker AT, Jalinous R, Freeston IL. "Non-invasive magnetic stimulation of human motor cortex". Lancet 1985; 1: 1106-1107

Bauer PR, de Goede AA, Stern WM, Pawley AD, Chowdhury FA, Helling RM et al. "Longinterval intracortical inhibition as biomarker for epilepsy: a transcranial magnetic stimulation study". Brain 2018; 141: 409-421

Ben-Ari Y, Holmes GL. "Effects of seizures on developmental processes in the immature brain". The Lancet Neurology 2006; 5: 1055-1063

Berg AT. "Identification of pharmacoresistant epilepsy". Neurol Clin 2009; 27: 1003-1013

Bonato C, Miniussi C, Rossini PM. "Transcranial magnetic stimulation and cortical evoked potentials: a TMS/EEG co-registration study". Clin Neurophysiol 2006; 117: 16991707

Casarotto S, Lauro LJR, Bellina V, Casali AG, Rosanova M, Pigorini A et al. "EEG responses to TMS are sensitive to changes in the perturbation parameters and repeatable over time". PLoS One 2010; 5: e10281

de Goede AA, Ter Braack EM, van Putten M. "Single and paired pulse transcranial magnetic stimulation in drug naive epilepsy". Clin Neurophysiol 2016; 127: 3140-3155

Del Felice A, Fiaschi A, Bongiovanni GL, Savazzi S, Manganotti P. "The sleep-deprived brain in normals and patients with juvenile myoclonic epilepsy: A perturbational approach to measuring cortical reactivity". Epilepsy Research 2011; 96: 123-131

Farrell JS, Gaxiola-Valdez I, Wolff MD, David LS, Dika HI, Geeraert BL et al. "Postictal behavioural impairments are due to a severe prolonged hypoperfusion/hypoxia event that is COX-2 dependent". Elife 2016; 5: e19352

Ferreri F, Pasqualetti P, Maatta S, Ponzo D, Ferrarelli F, Tononi G et al. "Human brain connectivity during single and paired pulse transcranial magnetic stimulation". Neuroimage 2011; 54: 90-102

Fisher RS, Acevedo C, Arzimanoglou A, Bogacz A, Cross JH, Elger CE et al. "ILAE official report: a practical clinical definition of epilepsy". Epilepsia 2014; 55: 475-482

Geut I, Weenink S, Knottnerus ILH, van Putten M. "Detecting interictal discharges in first seizure patients: ambulatory EEG or EEG after sleep deprivation?". Seizure 2017; 51: 52-54

Hallett M. "Transcranial magnetic stimulation and the human brain". Nature 2000; 406: $147-$ 150

Hanajima R, Ugawa Y, Terao Y, Sakai K, Furubayashi T, Machii K, Kanazawa I. "Pairedpulse magnetic stimulation of the human motor cortex - differences among Iwaves". Journal of Physiology 1998; 509: 607-618 
Hermann BP, Seidenberg M, Dow C, Jones J, Rutecki P, Bhattacharya A, Bell B. "Cognitive prognosis in chronic temporal lobe epilepsy". Ann Neurol 2006; 60: 80-87

Ilmoniemi RJ, Kičić D. "Methodology for combined TMS and EEG". Brain Topogr 2010; 22: 233-248

Ilmoniemi RJ, Virtanen J, Ruohonen J, Karhu J, Aronen HJ, Näätänen R, Katila T. "Neuronal responses to magnetic stimulation reveal cortical reactivity and connectivity". Neuroreport 1997; 8: 3537-3540

Inghilleri M, Berardelli A, Marchetti P, Manfredi M. "Effects of diazepam, baclofen and thiopental on the silent period evoked by transcranial magnetic stimulation in humans". Exp Brain Res 1996; 109: 467-472

Julkunen P, Säisänen L, Könönen M, Vanninen R, Kälviäinen R, Mervaala E. "TMS-EEG reveals impaired intracortical interactions and coherence in Unverricht-Lundborg type progressive myoclonus epilepsy (EPM1)". Epilepsy Research 2013; 106: 103 112

Kimiskidis VK, Tsimpiris A, Ryvlin P, Kalviainen R, Koutroumanidis M, Valentin A et al. "TMS combined with EEG in genetic generalized epilepsy: A phase II diagnostic accuracy study". Clin Neurophysiol 2017; 128: 367-381

Komssi S, Aronen HJ, Huttunen J, Kesäniemi M, Soinne L, Nikouline VV et al. "Ipsi- and contralateral EEG reactions to transcranial magnetic stimulation". Clin Neurophysiol 2002; 113: 175-184

Komssi S, Kähkönen S, Ilmoniemi RJ. "The effect of stimulus intensity on brain responses evoked by transcranial magnetic stimulation". Hum Brain Mapp 2004; 21: 154-164

Krumholz A, Wiebe S, Gronseth G, Shinnar S, Levisohn P, Ting T et al. "Practice Parameter: Evaluating an apparent unprovoked first seizure in adults (an evidence-based review): Report of the Quality Standards Subcommittee of the American Academy of Neurology and the American Epilepsy Society". Neurology 2007; 69: 1996-2007

Kujirai T, Caramia MD, Rothwell JC, Day BL, Thompson PD, Ferbert A et al. "Corticocortical inhibition in human motor cortex". Journal of Physiology 1993; 471: 501-519

Kwan P, Sander JW. "The natural history of epilepsy: an epidemiological view". J Neurol Neurosurg Psychiatry 2004; 75: 1376-1381

McCormick D, Contreras D. "On the cellular and network bases of epileptic seizures". Annu Rev Physiol 2001; 63: 815-846

McDonnell MN, Orekhov Y, Ziemann U. "The role of GABA(B) receptors in intracortical inhibition in the human motor cortex". Exp Brain Res 2006; 173: 86-93

Ngugi AK, Bottomley C, Kleinschmidt I, Sander JW, Newton CR. "Estimation of the burden of active and life-time epilepsy: a meta-analytic approach". Epilepsia 2010; 51: 883890 
Picot MC, Baldy-Moulinier M, Daures JP, Dujols P, Crespel A. "The prevalence of epilepsy and pharmacoresistant epilepsy in adults: a population-based study in a Western European country". Epilepsia 2008; 49: 1230-1238

Pierantozzi M, Marciani MG, Palmieri MG, Brusa L, Galati S, Caramia MD et al. "Effect of Vigabatrin on motor responses to transcranial magnetic stimulation: an effective tool to investigate in vivo GABAergic cortical inhibition in humans". Brain Res 2004; 1028: 1-8

Premoli I, Castellanos N, Rivolta D, Belardinelli P, Bajo R, Zipser C et al. "TMS-EEG signatures of GABAergic neurotransmission in the human cortex". J Neurosci 2014; 34: 5603-5612

Rossini PM, Barker AT, Berardelli A, Caramia MD, Caruso G, Cracco RQ et al. "Noninvasive electrical and magnetic stimulation of the brain, spinal cord and roots: basic principles and procedures for routine clinical application. Report of an IFCN committee". Electroencephalogr Clin Neurophysiol 1994; 91: 79-92

Rothwell JC. "Techniques and mechanisms of action of transcranial stimulation of the human motor cortex". Journal of Neuroscience Methods 1997; 75: 113-122

Schwenkreis P, Witscher K, Janssen F, Addo A, Dertwinkel R, Zenz M et al. "Influence of the N-methyl-D-aspartate antagonist memantine on human motor cortex excitability". Neurosci Lett 1999; 270: 137-140

Shafi MM, Vernet M, Klooster D, Chu CJ, Boric K, Barnard ME et al. "Physiological consequences of abnormal connectivity in a developmental epilepsy". Ann Neurol 2015; 77: 487-503

Valentin A, Arunachalam R, Mesquita-Rodrigues A, Garcia Seoane JJ, Richardson MP, Mills KR, Alarcon G. "Late EEG responses triggered by transcranial magnetic stimulation (TMS) in the evaluation of focal epilepsy". Epilepsia 2008; 49: 470-480

Werhahn KJ, Kunesch E, Noachtar S, Benecke R, Classen J. "Differential effects on motorcortical inhibition induced by blockade of GABA uptake in humans". Journal of Physiology 1999; 517: 591-597

Ziemann U, Chen R, Cohen LG, Hallett M. "Dextrometorphan decreases the excitability of the human motor cortex". Neurology 1998; 51: 1320-1324

Ziemann U, Reis J, Schwenkreis P, Rosanova M, Strafella A, Badawy R, Muller-Dahlhaus F. "TMS and drugs revisited 2014". Clin Neurophysiol 2015; 126: 1847-1868 


\section{Chapter 2 \\ Reduction of TMS induced artifacts in EEG using Principal Component Analysis}

Esther M. ter Braack, Benjamin J. de Jonge, Michel J.A.M. van Putten IEEE Transactions on Neural Systems and Rehabilitation Engineering 2013;21(3):376-382 


\section{Abstract}

Co-registration of TMS and EEG is a new, promising method for assessing cortical excitability and connectivity. Using this technique, a TMS evoked potential (TEP) can be induced and registered with the EEG. However, the TEP contains an early, short lasting artifact due to the magnetic pulse, and a second artifact, which depends on the location of stimulation and can last up to 40 milliseconds. Different causes for this second artifact have been suggested in literature. In this study, we used principal component analysis (PCA) to suppress both the first and second artifact in TMS-EEG data.

Single pulse TMS was applied at the motor and visual cortex in 18 healthy subjects. PCA using singular value decomposition was applied on single trials to suppress the artifactual components. A large artifact suppression was realized after the removal of the first 5 PCA components, thereby revealing early TEP peaks, with only a small suppression of later TEP components. The spatial distribution of the second artifact suggests that it is caused by electrode movement due to activation of the temporal musculature.

In conclusion, we showed that PCA can be used to reduce TMS-induced artifacts in EEG, thereby revealing components of the TMS evoked potential. 


\section{Introduction}

Co-registration of TMS (Barker et al. 1985) and EEG is a relatively new and promising method for assessing cortical excitability and connectivity. TMS-EEG provides researchers with the opportunity to stimulate the brain and directly measure the response of the stimulated area, without the need of detecting a peripheral response. When TMS is applied while recording EEG, a characteristic waveform the TMS evoked potential (TEP) - is induced in the EEG. The methodology of measuring and analyzing the TEP is similar to other event-related potential measurements, such as the visual or auditory evoked potential. Assuming that the stimulus always induces a specific response in the EEG, and considering all other brain activity as uncorrelated, the response can be extracted by averaging over several stimuli.

The TEP shows characteristic components at different latencies, and is most welldefined on electrode position Cz. Negative components at 15, 45 and $100 \mathrm{~ms}$ and positive components at 30,60 and $180 \mathrm{~ms}$ have been reported in several studies (Bonato et al. 2006; Casarotto et al. 2010; Esser et al. 2006; Ferreri et al. 2011; Ilmoniemi and Kičić 2010; Komssi et al. 2004; Levit-Binnun et al. 2010; Paus et al. 2001). Some authors describe even earlier peaks: a negative component at 7-10 ms and a positive component at 13-14 ms (Bonato et al. 2006; Ferreri et al. 2011).

To perform TMS-EEG measurements, special equipment is required, in particular to avoid saturation of the amplifier due to the strong electromagnetic pulse (Ilmoniemi and Kičić 2010). The two most common used techniques are using a sample-andhold circuit that short-circuits the amplifier input to ground for about $5 \mathrm{~ms}$ during the TMS pulse (Virtanen et al. 1999), or using an amplifier in which the sensitivity and operational range can be adapted (Bonato et al. 2006; Veniero et al. 2009), also referred to as a slew rate amplifier (Ives et al. 2006; Thut et al. 2005). Adapting these properties ensures that the amplifier does not saturate due to the TMS pulse. In the present study, we use a third technique: a DC amplifier, which has no capacitive elements and therefore does not saturate after a TMS pulse. The TMS pulse artifact measured by this amplifier is in the order of millivolts and lasts only approximately $5 \mathrm{~ms}$. Because of the short duration of this artifact, the early part of the TEP can be analyzed, as there is no interference with these early responses. In various cases, however, a second large amplitude artifact which slowly recovers is observed as well. This second artifact, starting from the time of the TMS pulse with a large 
positive peak at $5 \mathrm{~ms}$, a large negative peak at $10 \mathrm{~ms}$ and lasting up to tens of milliseconds (Mutanen et al. 2013), may obscure the early components of the TEP. As these early components reflect the excitability of the stimulated area, they have potential value as biomarkers for changes in cortical excitability, as may be present in epilepsy or stroke. Therefore, successful prevention of occurrence or removal of this second artifact is desirable.

Different causes for this second artifact have been suggested in literature. Because of its spatial distribution over the scalp and as the artifact occurs more frequently when temporal regions of the head are stimulated, a possible origin is the activation of the cranial muscles (Korhonen et al. 2011; Mäki and Ilmoniemi 2010; Mutanen et al. 2013). Alternatively, the artifact may be caused by the capacitive properties of the electrode-gel-skin circuits (Julkunen et al. 2008). A third possibility is the direct induction of currents in the electrode wires (Bender et al. 2005). Although precautions can be used to limit these contributions to the TEP artifacts, ranging from reducing the stimulus intensity and changing the tilt and rotation of the coil (Mutanen et al. 2013), using of needle electrodes (Julkunen et al. 2008) or rearranging electrode wires (Bender et al. 2005), in various experimental conditions significant artifacts remain present. Although the origin is not completely clear, it is agreed that the artifact and EEG signal come from independent sources, making independent or principal component analysis (ICA or PCA) ideal techniques to suppress this artifact.

A few papers have proposed signal processing techniques to remove the artifact from TMS-EEG recordings, such as Kalman filters (Balduzzo et al. 2003) or ICA (Hamidi et al. 2010; Iwahashi et al. 2008; Korhonen et al. 2011). However, these methods were aimed only at the first TMS artifact (Balduzzo et al. 2003; Hamidi et al. 2010), or did not discuss a possible effect on the physiological waveforms of the TEP when artifactual components were removed (Iwahashi et al. 2008). Only one study showed that PCA can be used successfully to remove the second artifact (Mäki and Ilmoniemi 2010). However, it was combined with a topographic projection method and applied to a limited number of subjects $(n=3)$ (Mäki and Ilmoniemi 2010). In addition, the TEP that was obtained after artifact removal was very low in amplitude, because also parts of the TEP (with the same topography as the second artifact) were removed by PCA, although the amount of suppression was exactly known. 
We present a method to reduce both the first and second artifact in TMS-EEG data using only PCA, evaluated in a larger number of subjects. In our approach, no assumptions are made about the topographical distribution of the artifact. Ideally, the artifact removal technique should only reduce the TMS artifact, thereby revealing early components of the TEP, without a significant effect on the later components of the TEP. Therefore, we also evaluated if PCA attenuated later parts of the response, which were not corrupted by artifacts.

\section{Materials and methods Subjects}

Eighteen healthy subjects (11 males, mean age 28 years, all right-handed) participated in this study after giving written informed consent. The experimental protocol was approved by the local ethical committee (Medisch Spectrum Twente) and was in accordance with the declaration of Helsinki.

\section{Stimulation}

Single biphasic TMS pulses, with pulse duration of $400 \mu$ s and inter-pulse interval of 4 seconds, were delivered manually using a $70 \mathrm{~mm}$ figure-of-eight air film coil and a Magstim Rapid ${ }^{2}$ stimulator. The coil was placed tangentially with the handle pointing backwards and laterally at an angle $45^{\circ}$ away from the midline over four targets: the hot-spot of the abductor digiti minimi muscle (ADM) in the right and left motor cortex; and Brodmann area 19 in the right and left hemisphere. The maximum stimulator output was 1.5 tesla; stimulation intensity for the targets in the left hemisphere was set at $110 \%$ of the resting motor threshold (RMT) of the left ADM hot spot and at $110 \%$ RMT of the right ADM hot spot for the targets in the right hemisphere. The motor threshold was defined as the lowest stimulus intensity that produced at least five MEPs of at least $50 \mu \mathrm{V}$ out of ten consecutive stimuli (Rossini et al. 1994). There were 5 sessions for every subject; in each session we applied 75 pulses at all four targets.

\section{TMS targeting}

Positioning of the coil was achieved using a robot-navigated system (Advanced Neuro Technology, Enschede, Netherlands). A headband carrying four passive reflective markers was fixed to the head of the subject and tracked by a Polaris infrared camera system (Northern Digital Inc., Waterloo, Ontario, Canada). The robot and the tracking system were registered to a common coordinate system using 
a calibration procedure. The robot-guided TMS coil was added to the coordinate system by registration of three reference positions on the coil using a tracking pointer. In all subjects, a 1.5 tesla MRI scan of the head was available. The MRI scan was used to create a subject-specific head model; this model was then registered to the subject's head and the coordinate system by collecting three landmarks and 300 additional points on the scalp with a tracking pointer.

\section{EEG and EMG recording during TMS}

The EEG was recorded continuously during TMS using a DC amplifier and a TMScompatible 64-electrode cap (ANT, Enschede, Netherlands). Impedances were kept below $5 \mathrm{kOhm}$. The ground electrode was placed between electrode positions $\mathrm{Fz}$ and Fpz. We used a common average reference for the recordings. To determine the RMT, we recorded the EMG using an additional amplifier (TMSi, Oldenzaal, Netherlands) connected to the EEG amplifier, ensuring synchronized measurements. Surface electrodes were placed in a belly-tendon montage over the ADM muscle. The ground electrode was placed on the dorsal side of the wrist. EEG and EMG data were low-pass filtered with an anti-aliasing filter with a cut-off frequency of $550 \mathrm{~Hz}$ and sampled at $2048 \mathrm{~Hz}$.

\section{EEG analysis}

We assumed that no differences in artifact were present when recording during different sessions, therefore all 375 applied pulses per target per subject were used for analysis. The recorded EEG data were divided in trials of 4 seconds (2 seconds before and after a TMS pulse). We used the common average reference for analyzing the data. Trials with eye blinks were automatically detected and these were rejected for further analysis. Principal Component Analysis using Singular Value Decomposition was used to decompose the data into different components.

\section{PCA}

Principal Component Analysis (Bestmann 2008) is a well-established multivariate data technique which finds the direction in the data with most variation. It is expected that the direction with most variation contains the TMS induced artifacts, because of the large amplitude difference between artifact and EEG signals. 
Initially, we subtracted the mean from dataset $\mathbf{X}$, which contains the EEG data, the rows being the number of electrodes, and the columns being the number of data points. The covariance matrix $\mathbf{C}$ was then calculated, given by

$$
\mathbf{C}=\frac{1}{n} \mathbf{X} \mathbf{X}^{T}
$$

in which $T$ means transposed. From the covariance matrix $\mathbf{C}$ the eigenvectors and eigenvalues are calculated and sorted according to their eigenvalue. Singular Value Decomposition is now used to decompose dataset $\mathbf{X}$ in matrices $\mathbf{U}, \mathbf{S}$ and $\mathbf{V}$ :

$$
\mathbf{X}=\mathbf{U S V}^{T} \text {. }
$$

Orthogonal matrix $\mathbf{U}$ captures the eigenvectors calculated in the previous step in each column; $\mathbf{S}$ contains the singular values (square root of eigenvalues of $\mathbf{X X}^{\mathrm{T}}$ ) and orthogonal matrix $\mathbf{V}$ contains the eigenvectors of $\mathbf{X}^{\mathbf{T}} \mathbf{X}$.

Matrix $\mathbf{S}$ is ordered from high to low values. The highest value describes the component captured in the eigenvector with the highest variation. We performed PCA using 40 calculated components on each individual trial. Using the eigenvector matrix $\mathbf{U}$ and singular values in $\mathbf{S}$, the number of components to be removed can be selected.

The data can then be reconstructed with only the remaining principal components ( $\tilde{\mathbf{U}})$, which are ideally the components that do not describe the artifact:

$$
\mathbf{X}_{\text {corrected }}=\tilde{\mathbf{U}}{ }^{*} \tilde{\mathbf{U}}^{-1}{ }^{*} \mathbf{X}_{\text {original }} .
$$

For all subjects, the first 20 principal components were removed, one component at a time. After removing each consecutive component, the TEP was obtained by averaging over trials.

\section{Evaluation of artifact removal}

For evaluation of the effect of the removal of the PCA components on the amplitude of the artifact and TEP, we visually detected the artifact and TEP components for every consecutive PCA component that was removed. We chose two electrodes to evaluate the artifact removal, one directly at the site of TMS (which was C3 for left motor cortex stimulation and $\mathrm{C} 4$ for right motor cortex stimulation) and one in an area where the TEP is the most well-defined $(\mathrm{Cz})$. The first large - positive or negative - peak between $0 \mathrm{~ms}$ and $5 \mathrm{~ms}$ after TMS administration represents the first artifact. The large positive peak between $5 \mathrm{~ms}$ and $10 \mathrm{~ms}$ was used as a quantitative measure for the second artifact. Changes in the absolute values of both artifacts for the electrode of stimulation, $\mathrm{C} 3$ or $\mathrm{C} 4$, using the unfiltered signal, were used as 
performance measures. In addition, we also analyzed the first artifact at electrode Cz. We then applied a low-pass Butterworth filter with a cut-off frequency of 150 $\mathrm{Hz}$ and visually analyzed the TEP at electrode Cz. The P30, N45, P60 and N100 components of the TEP were visually assessed, both for left and right motor cortex stimulation. We subsequently calculated the peak-to-peak amplitudes P30-N45 (referred to as P30), P60-N45 (referred to as P60) and P60-N100 (referred to as N100). All initial amplitudes (artifact and TEP components) were normalized to 1. The amplitude changes were subsequently evaluated as a function of the number of removed principal components.

\section{Results}

A first, large artifact, with a duration of approximately $5 \mathrm{~ms}$, was seen when stimulating the motor cortex and Brodmann area 19. In 16 out of 18 subjects, an additional artifact was visible after stimulation of the motor cortex, which was not present when Brodmann area 19 was stimulated. This second artifact was located temporal from the stimulated target. The topography and average power of the first and second artifact are illustrated in figure 2.1, showing a grand average of these 16 subjects.
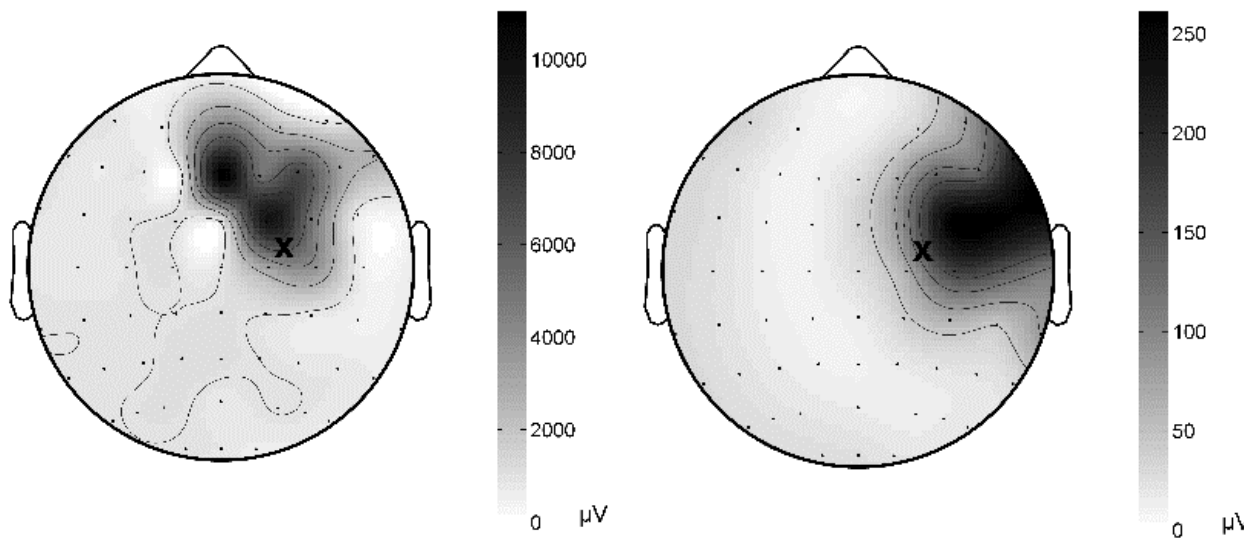

Figure 2.1. Topoplots of signal power for the first (left) and second (right) artifact, after stimulation at the right motor cortex. Note the large difference in amplitude between the first and second artifact. When Brodmann area 19 was stimulated, no second artifact was observed. Grand average of 16 subjects. X denotes the stimulus position. 
In figure 2.2, responses in channels $\mathrm{C} 4$ and $\mathrm{Cz}$ from two subjects are shown before and after PCA correction. In one of these subjects a large second artifact was seen: the first artifact ends at approximately $5 \mathrm{~ms}$; the second artifact shows a positive peak at $8 \mathrm{~ms}$ and a negative peak at $10 \mathrm{~ms}$, after which it slowly recovers. Since the TMS coil was positioned just above electrode $\mathrm{C} 4$, this is one of the channels that shows the largest second artifact. The TEP, however, is most well-defined at electrode Cz. TEP components at 30, 45, 60 and $100 \mathrm{~ms}$ are visible at this electrode in the uncorrected response. For the two subjects without a second artifact, earlier components at $\mathrm{Cz}$ can be identified in the uncorrected signal, but for subjects with a large second artifact, these only become visible after removing 3-4 principal components. These early peaks are similar in latency for all subjects (N10 - P15 $\mathrm{N} 20$ for $\mathrm{Cz}$ ) and correspond to early TEP components reported in literature (Bonato et al. 2006; Ferreri et al. 2011). At electrode C4, different early TEP components become visible as well (N10 - P15 - N18). After removal of five principal components, the amplitude of both first and second artifact is greatly reduced, although there is some residual left. After rejection of more PCA components, the TEP components at 30, 45 and $60 \mathrm{~ms}$ also reduce in amplitude. Latencies of these peaks do not change after PCA correction. 
Subject 1
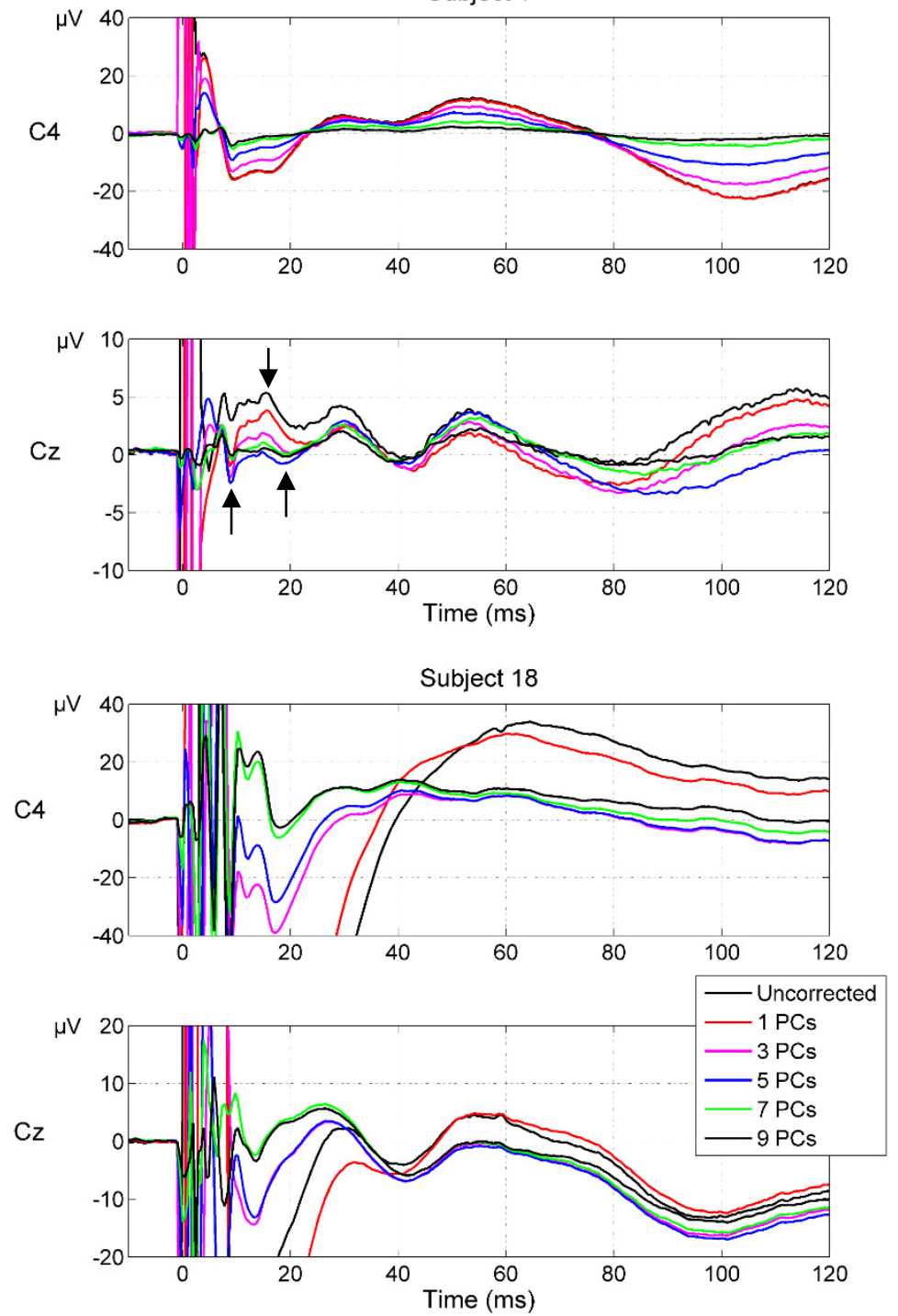

Figure 2.2. The PCA performance is shown for the unfiltered recordings at electrode $\mathrm{C} 4$ and $\mathrm{Cz}$ in two subjects. Note the different scaling at the y-axes. Subject 1 (top figures) did not show a second artifact, while subject 18 (bottom figures) had a large second artifact. TEP components at 30, 45 and $60 \mathrm{~ms}$ are clearly visible at $\mathrm{Cz}$ in the uncorrected response in both subjects; earlier components only become visible after PCA correction in subject 18, while in subject 1 these could be identified without PCA (indicated by the arrows). TMS was targeted at the right motor cortex, above electrode position $\mathrm{C} 4$. 
In figure 2.3 the TEP at all electrodes is shown for a single subject after stimulation of the left motor cortex. The effects of removing the first 10 principal components on the TEP for the same subject is shown in figure 2.4. The artifacts are greatly reduced, although there is still some residual artifact in the frontotemporal electrodes visible. These remaining artifacts disappear when more components are removed, but then the TEP also reduces in amplitude; this trade-off is shown in figure 2.5.

The effect of removing 1 to 20 principal components from the data obtained in 16 subjects after stimulating the right motor cortex is shown in figure 2.5 . The reduction in amplitude of the first artifact is the largest after removal of the first component, resulting in a reduction of amplitude to about 0.25 of the initial value for electrode $\mathrm{Cz}$ and $\mathrm{C} 4$. However, because the original amplitude is very high, the remaining artifact is still significantly larger than the TEP components. After removing approximately 5 principal components, the artifact amplitude approaches zero. Removing the second artifact proves to be more difficult, after five PCA components, the amplitude is below $40 \%$ of the initial value. For the TEP components, there is a large variation between subjects in the amplitude change. However, on average the amplitude of the P60 and N100 stays within a 20\% decrease if the first 5 components are removed. Because the second artifact lasts up to 30-40 $\mathrm{ms}$, the presence of the $\mathrm{P} 30$ becomes more pronounced after PCA components are removed, leading in some subjects to an increasing P30 amplitude. The large variation in P30 amplitude between subjects is a result of the differences in the amount of second artifact that is present in each individual subject, with corresponding inter-individual differences in the amount of reduction after PCA. 


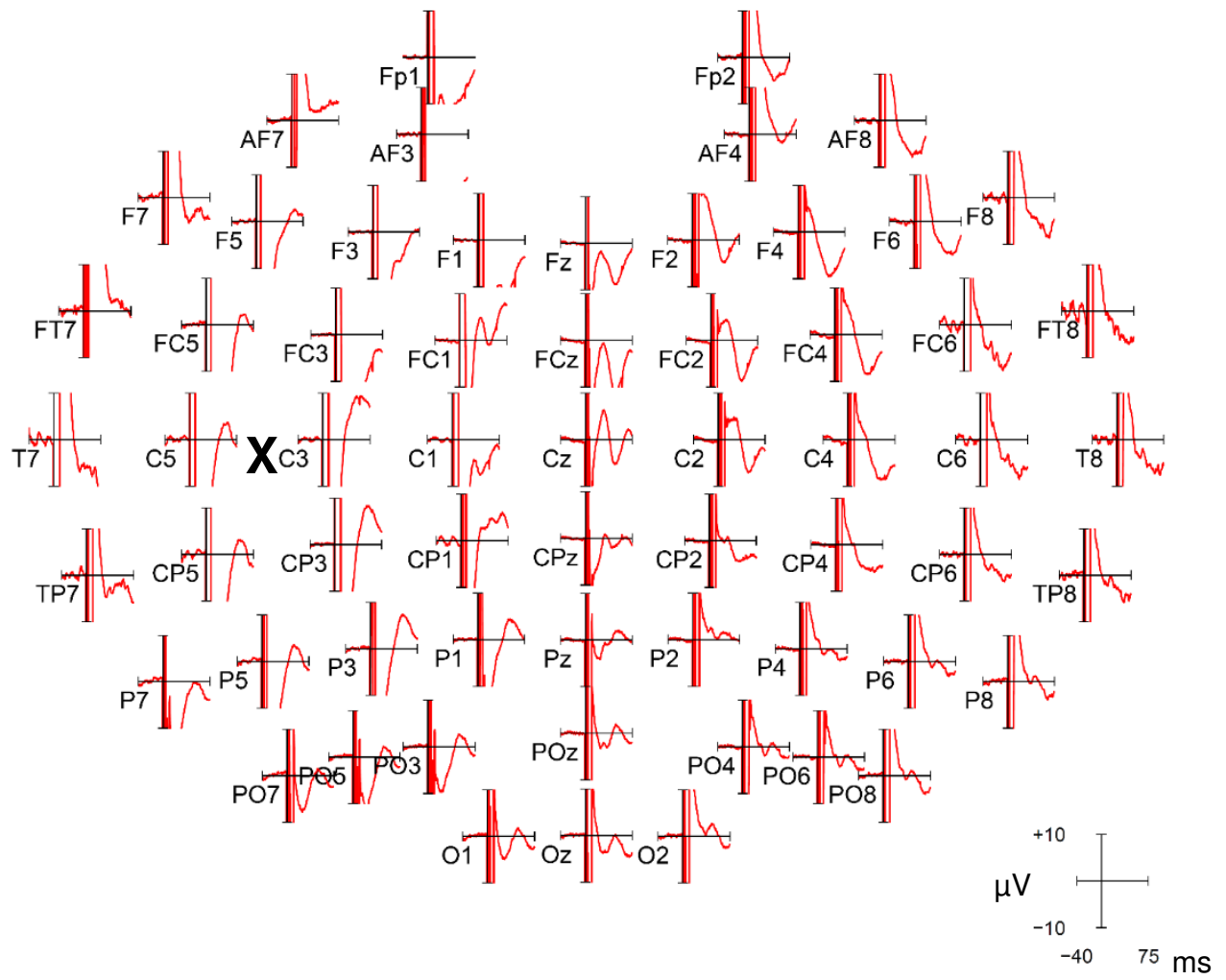

Figure 2.3. TEP before artifact correction using PCA in a subject 10. The left motor cortex (around C3) was targeted with TMS. 


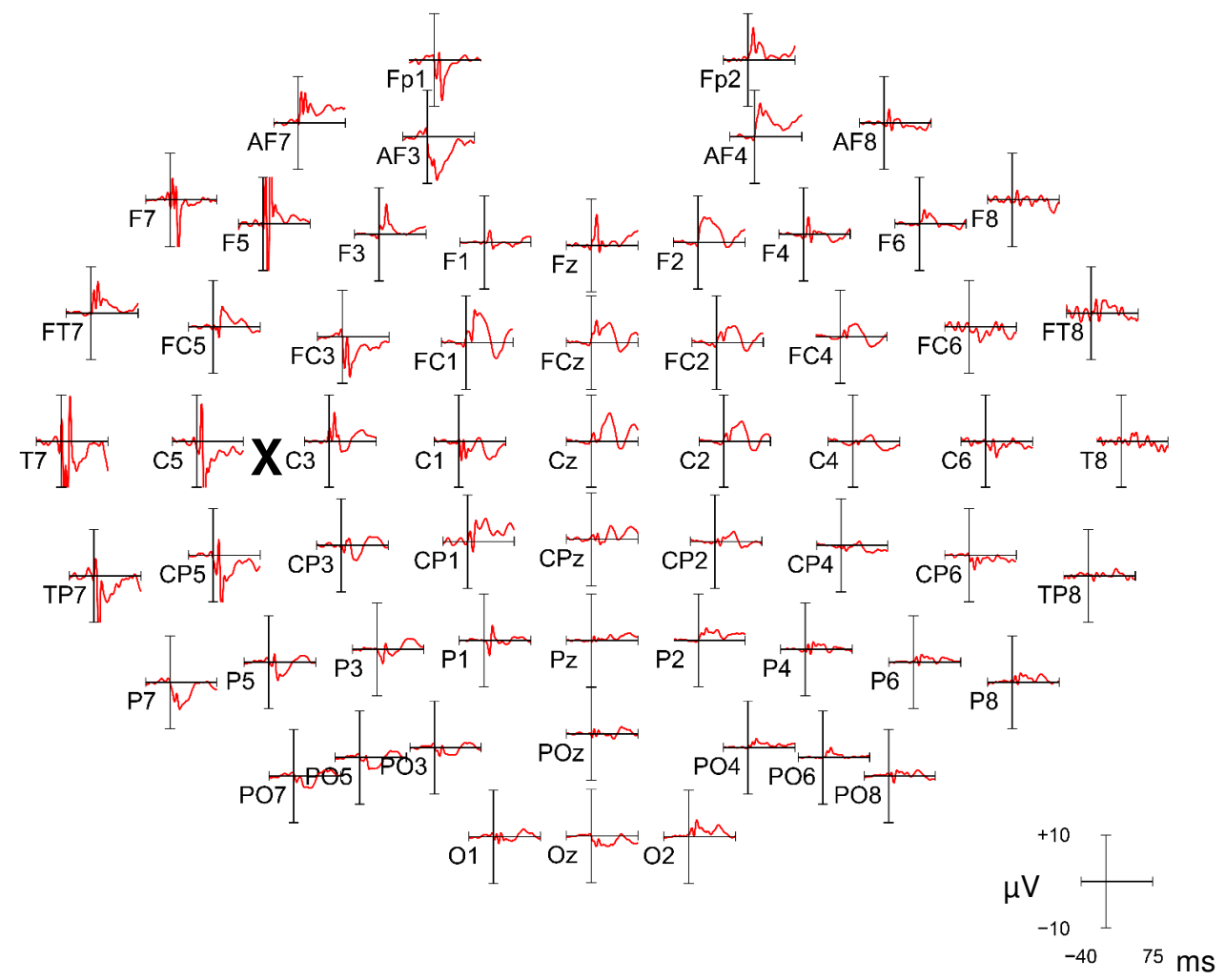

Figure 2.4. TEP after removing 10 principal components in subject 10. The left motor cortex (around C3) was targeted with TMS. The artifact is greatly reduced as compared to figure 2.3 , while the main components of the TEP are preserved. However, there is still some artifact remaining at the left frontotemporal electrodes. 

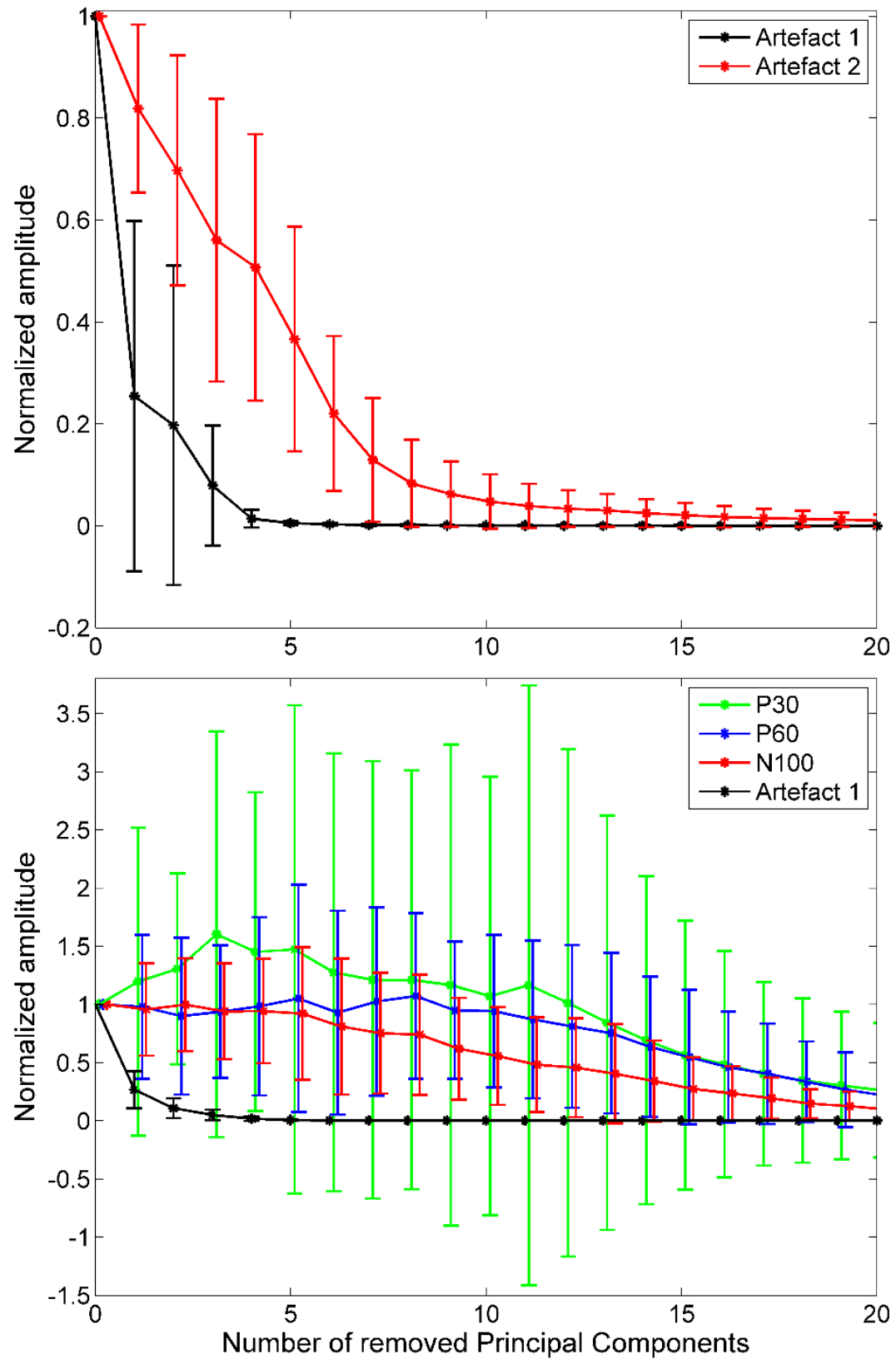

Figure 2.5. Normalized amplitude of the first and second artifact for electrode C4 (left) and of the first artifact, P30, P60 and N100 as a function of the number of removed components for electrode $\mathrm{Cz}$ (right). Errorbars represent the standard deviation. Grand average of 18 subjects. TMS was targeted at the right motor cortex. 


\section{Discussion}

TMS-EEG is a promising technique to explore cortical excitability by analysis of the different waveforms present in the TMS-evoked potential. However, in particular during the first $30 \mathrm{~ms}$, artifacts may be present that obscure the interpretation of early responses. Here, we explore the nature of the TMS artifacts, and if PCA is a suitable method to remove the various artifactual components.

The amplitudes of both first (0-5 ms) and second (5-10 ms) TMS artifact were strongly reduced by removing components using PCA. With approximately five components removed, the later responses of the TEP (P60 and N100) stayed within a $20 \%$ decrease. With five removed components, the reduction in artifact amplitude is sufficiently large to allow further signal processing, such as filtering and analysis of the various latencies of the TEP waveforms. In all subjects, this cut-off number of approximately five components was found.

The number of components to be removed has to be considered carefully for each individual subject: with too few components the artifact is not reduced enough to see the early TEP peaks, and with too many components the later components of the TEP become very small. In addition, the electrode of interest is also important. For the area directly surrounding the TMS targeting, more principal components have to be removed to obtain a sufficient artifact reduction, while for electrodes further away from the TMS target the same result is obtained with only a few removed components. Although the second artifact is reduced less than the first artifact, the artifact suppression by removing five principal components is strong enough to reveal early TEP components, which was the aim of this study. Computation time was 5-6 minutes for one target in a single subject.

The spatial distribution of the evoked potentials showed that when the motor cortex was stimulated, the first artifact is located more frontal to the target, while the second artifact can be found at the temporal areas. When the occipital region at Brodmann area 19 was stimulated, the first artifact was situated just beneath the target, and no second artifact was observed. These findings make a muscular origin for the second artifact plausible. However, the duration of the artifact is too long for a compound muscle action potential, which lasts only a few milliseconds. A possible explanation is that the artifact is not the muscle activation itself, but a subsequent movement of the electrodes that are located above the muscle, induced by the muscle contraction. 
This would also explain why some authors achieved better results with needle electrodes (Julkunen et al. 2008), because then the amount of electrode movement is presumably limited.

Another possible cause for the second artifact is an induction effect in the wires of the EEG cap (Bender et al. 2005); the specific distribution of the artifact may be caused by a different position of the wires at the temporal sides of our EEG cap. Indeed, in our subjects we do not observe the second artifact when stimulating the top of the head or the occipital regions. In measurements using a phantom with a TMS-compatible EEG cap, stimulating at different locations, a second artifact was never observed, providing further evidence that wiring is not responsible for the second artifact. A similar technique was already used in literature to investigate the first TMS artifact (Veniero et al. 2009), and Mutanen et al. also showed that the second artifact was not present using a phantom head (Mutanen et al. 2013). Although the contribution of a capacitive effect of the electrode-skin interface (Julkunen et al. 2008) cannot be excluded in our experiments, this is unlikely as well, as the second artifact was not present during stimulation over the occipital electrodes.

Recently, PCA combined with a topography-based method has been reported to be able to completely remove TMS induced artifacts, which were assumed to originate from a muscle contraction (Mäki and Ilmoniemi 2010). These authors determined the components to be taken out based on the topography of this muscle activity, acknowledging the fact that also brain activity with the same topography is strongly suppressed. This implies that brain activity generated by sources just underneath the coil - most likely the areas activated first by the pulse, and therefore the areas responsible for the earliest components of the TEP - will be reduced. This is especially important when the area activated by TMS is located at the site where the artifact is most visible, for example at temporal regions, although the amount of attenuation is known. The TEP components were found to be significant in the calculated global mean field potential (GMFA) after artifact reduction, but the presence of early TEP components before and after artifact reduction was not shown.

In our implementation, no assumptions about topography are made, resulting in an evenly reduction of the artifact (and TEP when too many components are removed) over the cortex. Furthermore, in the approach described in (Mäki and Ilmoniemi 2010), the PCA components were calculated after initial averaging of the TEP, while 
we apply PCA on single trials. At present, it is not clear which method is most suitable. An additional improvement in topography-based PCA may result from removing less principal components.

After applying PCA, removing approximately 5 principal components results in an artifact suppression of more than 10 times. Early components of the TEP, which were initially obscured by the second artifact, are revealed using this technique. There were only minor effects on the later components of the TEP, except for the P30 which showed large variations in amplitude, probably because this TEP component is largely affected by the second artifact. Although both artifacts are strongly reduced by PCA, complete removal cannot be guaranteed with our approach. The low-amplitude peaks that remain, may still be small remainders of the artifacts, this may indeed be true for the negative peak around $10 \mathrm{~ms}$ in $\mathrm{Cz}$, which is at the same latency as the large negative peak in the second artifact (Mutanen et al. 2013). On the other hand, it is likely that reducing the amplitude of both artifacts did reveal true brain responses that were initially hidden, especially because latencies of these emerging early peaks - also the N10 - were similar to early TEP components reported in literature (Bonato et al. 2006; Ferreri et al. 2011). In these studies, no second artifact was observed in the data, and no artifact rejection technique was used that could have induced small fluctuations resembling TEP components. In any case, small effects on the response itself have to be taken into account as well when analyzing the data further, especially when applying source analysis.

In conclusion, the TEP response obtained in TMS-EEG measurements contains an early artifact due to the magnetic pulse, and a second artifact. The second artifact depends on the location of stimulation, and is most likely caused by muscle activation due to the TMS pulse, possibly followed by electrode movements. We showed that PCA can be used to reduce TMS artifacts so that interpretation of early responses is possible, without the need for additional or complex signal analysis methods. This is particularly relevant when the TMS is targeted at the temporal regions of the brain, for example in research concerning auditory or speech functions, or when the seizure onset zone is stimulated in patients with temporal lobe epilepsy. 


\section{References}

Balduzzo M, Milone FF, Minelli TA, Pittaro-Cadore I, Turicchia L. "Mathematical phenomenology of neural stimulation by periodic fields". Nonlinear Dynamics Psychol Life Sci 2003; 7: 115-137

Barker AT, Jalinous R, Freeston IL. "Non-invasive magnetic stimulation of human motor cortex". Lancet 1985; 1: 1106-1107

Bender S, Basseler K, Sebastian I, Resch F, Kammer T, Oelkers-Ax R, Weisbrod M. "Electroencephalographic response to transcranial magnetic stimulation in children: Evidence for giant inhibitory potentials". Ann Neurol 2005; 58: 58-67

Bestmann S. "The physiological basis of transcranial magnetic stimulation". Trends Cogn Sci 2008; 12: 81-83

Bonato C, Miniussi C, Rossini PM. "Transcranial magnetic stimulation and cortical evoked potentials: a TMS/EEG co-registration study". Clin Neurophysiol 2006; 117: 16991707

Casarotto S, Lauro LJR, Bellina V, Casali AG, Rosanova M, Pigorini A et al. "EEG responses to TMS are sensitive to changes in the perturbation parameters and repeatable over time". PLoS One 2010; 5: e10281

Esser SK, Huber R, Massimini M, Peterson MJ, Ferrarelli F, Tononi G. "A direct demonstration of cortical LTP in humans: a combined TMS/EEG study". Brain Res Bull 2006; 69: 86-94

Ferreri F, Pasqualetti P, Määttä S, Ponzo D, Ferrarelli F, Tononi G et al. "Human brain connectivity during single and paired pulse transcranial magnetic stimulation". Neuroimage 2011; 54: 90-102

Hamidi M, Slagter HA, Tononi G, Postle BR. "Brain responses evoked by high-frequency repetitive transcranial magnetic stimulation: an event-related potential study". Brain Stimul 2010; 3: 2-14

Ilmoniemi RJ, Kičić D. "Methodology for combined TMS and EEG". Brain Topogr 2010; 22: $233-248$

Ives JR, Rotenberg A, Poma R, Thut G, Pascual-Leone A. "Electroencephalographic recording during transcranial magnetic stimulation in humans and animals". Clin Neurophysiol 2006; 117: 1870-1875

Iwahashi M, Arimatsu T, Ueno S, Iramina K. "Differences in evoked EEG by transcranial magnetic stimulation at various stimulus points on the head". Conf Proc IEEE Eng Med Biol Soc 2008; 2008: 2570-2573

Julkunen P, Pääkkönen A, Hukkanen T, Könönen M, Tiihonen P, Vanhatalo S, Karhu J. "Efficient reduction of stimulus artefact in TMS-EEG by epithelial short-circuiting by mini-punctures". Clin Neurophysiol 2008; 119: 475-481

Komssi S, Kähkönen S, Ilmoniemi RJ. "The effect of stimulus intensity on brain responses evoked by transcranial magnetic stimulation". Hum Brain Mapp 2004; 21: 154-164 
Korhonen RJ, Hernandez-Pavon JC, Metsomaa J, Mäki H, Ilmoniemi RJ, Sarvas J. "Removal of large muscle artifacts from transcranial magnetic stimulation-evoked EEG by independent component analysis". Med Biol Eng Comput 2011; 49: 397-407

Levit-Binnun N, Litvak V, Pratt H, Moses E, Zaroor M, Peled A. "Differences in TMSevoked responses between schizophrenia patients and healthy controls can be observed without a dedicated EEG system". Clin Neurophysiol 2010; 121: 332-339

Mäki H, Ilmoniemi RJ. "Projecting out muscle artifacts from TMS-evoked EEG". Neuroimage 2010; 54: 2706-2710

Mutanen T, Mäki H, Ilmoniemi RJ. "The Effect of Stimulus Parameters on TMS-EEG Muscle Artifacts". Brain Stimulation 2013; 6: 371-376

Paus T, Sipila PK, Strafella AP. "Synchronization of neuronal activity in the human primary motor cortex by transcranial magnetic stimulation: an EEG study". J Neurophysiol 2001; 86: 1983-1990

Rossini PM, Barker AT, Berardelli A, Caramia MD, Caruso G, Cracco RQ et al. "Noninvasive electrical and magnetic stimulation of the brain, spinal cord and roots: basic principles and procedures for routine clinical application. Report of an IFCN committee". Electroencephalogr Clin Neurophysiol 1994; 91: 79-92

Thut G, Ives JR, Kampmann F, Pastor MA, Pascual-Leone A. "A new device and protocol for combining TMS and online recordings of EEG and evoked potentials". J Neurosci Methods 2005; 141: 207-217

Veniero D, Bortoletto M, Miniussi C. "TMS-EEG co-registration: on TMS-induced artifact". Clin Neurophysiol 2009; 120: 1392-1399

Virtanen J, Ruohonen J, Naatanen R, Ilmoniemi RJ. "Instrumentation for the measurement of electric brain responses to transcranial magnetic stimulation". Med Biol Eng Comput 1999; 37: 322-326 


\section{Chapter 3}

\section{Masking the Auditory Evoked Potential in TMS- EEG: a comparison of various methods}

Esther M. ter Braack, Cecile C. de Vos, Michel J.A.M. van Putten Brain Topography 2015;28:520-528 


\section{Abstract}

There is growing interest in combining transcranial magnetic stimulation (TMS) with electroencephalography (EEG). Because TMS pulses are accompanied by a clicking sound, it is very likely that part of the response in the EEG consists of an auditory evoked potential (AEP). Different methods have been applied to mask the sound of TMS. However, it is unclear which masking method is most effective in reducing the AEP. In this study we explore the presumed contribution of the AEP to the response and evaluate different ways to mask the TMS clicking sound. Twelve healthy subjects and one completely deaf subject participated in this study. Eight different masking conditions were evaluated in nine hearing subjects. The amplitude of the N100-P180 complex was compared between the different masking conditions. We were not able to completely suppress the N100-P180 when the coil was placed on top of the head. Using an earmuff or exposing the subjects to white or adapted noise caused a small but significant reduction in N100-P180 amplitude, but the largest reduction was achieved when combining a layer of foam, placed between coil and head, with white or adapted noise. The deaf subject also showed a N100-P180 complex. We conclude that both the TMS clicking sound and cortical activation by the magnetic pulse contribute to the N100-P180 amplitude. 


\section{Introduction}

Transcranial magnetic stimulation (TMS) is a widely used technique to noninvasively activate the human cortex. After the introduction in the mid-eighties (Barker et al. 1985), TMS has developed into a method that is used to study motor cortical excitation (resting motor threshold) and inhibition (short and long interval cortical inhibition) by measuring modulation of the motor evoked potential. In addition, various diagnostic and therapeutic applications have emerged, including determining the central motor conduction time for diagnosing spinal cord compression, amyotrophic lateral sclerosis or multiple sclerosis (Chen et al. 2008), and applying repetitive TMS for treating depression (Padberg and George 2009).

Recently, there is a growing interest in combining TMS with electroencephalography (EEG), because it is a more direct measurement of the brain's reaction to a pulse than the motor evoked potential. In addition, the response of other areas than the motor cortex can be studied with TMS-EEG. When TMS is applied while recording EEG, a characteristic waveform - the TMS evoked potential (TEP) - is induced in the EEG (Ilmoniemi and Kičić 2010). The methodology of measuring and analyzing the TEP is similar to other event-related potential measurements, such as the visual or auditory evoked potential. Assuming that the stimulus always induces a specific response in the EEG, and considering all other brain activity as uncorrelated, the response can be extracted by averaging over several stimuli. Combining TMS with EEG can give more insight into the connectivity between different brain areas, by evaluating the TEP at different electrode positions while stimulating a specific target.

The response shows characteristic components at different latencies. Negative components at 15, 45 and $100 \mathrm{~ms}$ and positive components at 30,60 and $180 \mathrm{~ms}$, which are specific for the TEP at electrode $\mathrm{Cz}$ after motor cortex stimulation, have been reported in several studies (Bonato et al. 2006; Esser et al. 2006; Ferreri et al. 2011; Ilmoniemi and Kičić 2010; Komssi et al. 2004; Levit-Binnun et al. 2010; Paus et al. 2001; ter Braack et al. 2013). The TEP has shown to be reproducible within a one-week interval (Lioumis et al. 2009) and changes shape when different parts of the cortex are stimulated (Casarotto et al. 2010). The physiological processes responsible for the different components are still largely unknown (Bonato et al. 2006), although some studies suggested that the N100 reflects cortical inhibitory processes (Kičić et al. 2008; Nikulin et al. 2003). The variation in the amplitude of 
the motor evoked potential (MEP) has also been shown to correspond to variations in the amplitude of earlier TEP peaks (N15 and P30) (Mäki and Ilmoniemi 2010).

Because a TMS pulse is accompanied by a clicking sound, it is very likely that at least part of the TEP consists of an auditory evoked potential (AEP) (Ilmoniemi and Kičić 2010; Miniussi and Thut 2010; Nikouline et al. 1999). The AEP consists of several peaks (Picton et al. 1974), of which the long latency components P50, N100 and P180 could potentially interfere with the TEP. At present, it is not completely known which components of the TEP may be corrupted by components of the AEP. Although it has been shown that especially the N100 and P180 of the TEP are strongly correlated to auditory stimuli (Nikouline et al. 1999; Tiitinen et al. 1999), the magnitude of the contribution of the TMS click to the TEP is still unclear.

To reduce the presence of the AEP, different methods have been applied to mask the sound of the TMS pulse. In some studies, earplugs alone (Julkunen et al. 2008; Kähkönen et al. 2001; Kähkönen et al. 2005a; Kähkönen et al. 2005b), or a combination of earplugs with an earmuff (Bikmullina et al. 2009; Kičić et al. 2008; Nikulin et al. 2003), have been used. Other studies report the administration of sound through (regular or inserted) headphones to make sure that the subject does not hear the TMS click. In these studies, either white noise (Fuggetta et al. 2005; Hamidi et al. 2010; Levit-Binnun et al. 2010; Paus et al. 2001; Veniero et al. 2010; Werf and Paus 2006) or noise created from the TMS click itself (adapted noise) (Ferrarelli et al. 2010) was used. It has been recognized that sound also travels via bone conduction and not via air alone (Nikouline et al. 1999). Therefore, in addition to playing white or adapted noise, different authors placed a thin layer of foam between the TMS coil and the head of the subject (Casali et al. 2010; Casarotto et al. 2010; Esser et al. 2006; Ferrarelli et al. 2010; Mäki and Ilmoniemi 2010; Massimini et al. 2005; Massimini et al. 2010; Rosanova et al. 2009).

Despite these various studies, it is still not clear which sound masking method is the most effective in reducing the AEP and which TEP components are possibly contaminated by it. In this study we further explore the presumed contribution of the AEP to the TEP, and evaluate different ways to mask the TMS clicking sound by comparing the presence of the P50 and the amplitude of the N100-P180 complex in both normal hearing subjects and a completely deaf person. 


\section{Materials and Methods \\ Subjects}

Twelve healthy subjects ( 8 males, mean age 25 years, range 23-27 years, 11 righthanded) participated in this study after given written informed consent. We also included one subject who was born completely deaf ( 1 female, age 37 years, righthanded), which was confirmed with an audiogram. Whether the deafness was due to central or peripheral deficits has not been determined. The experimental protocol was approved by the local ethics committee (Medisch Spectrum Twente) and was in accordance with the declaration of Helsinki. We followed the guidelines for the use of TMS in clinical practice and research (Rossi et al. 2009).

\section{Experimental conditions}

Subjects were seated in a chair, with their hands pronated in a relaxed position. They kept their eyes open and focussed on a marked point on the wall. There were two experiments.

In experiment 1 , we evaluated the effect of 8 different masking conditions on the TEP in nine hearing subjects (subject 10 only participated in experiment 2 , subjects 11 and 12 only participated in the control measurements, see below). These 8 conditions, in which earplugs, an earmuff, two types of noise and a layer of foam were combined, are listed in table 3.1. The acronyms used are NM (no masking), E (earplugs), EE (earplugs/earmuff), EEF (earplugs/earmuff/foam), EW (earplugs/white noise), EWF (earplugs/white noise/foam), EA (earplugs/adapted noise) and EAF (earplugs/adapted noise/foam). The white and adapted noise were applied using external headphones. In addition, we tested the influence of various masking methods on the motor threshold, by determining the threshold in conditions NM, EA and EAF (see table 3.1). This threshold was also determined without masking, but with the layer of foam between coil and head (condition F, see table 3.1). The motor threshold was defined as the lowest stimulus intensity which produced at least five MEPs of $50 \mu \mathrm{V}$ out of ten consecutive stimuli (Rossini et al. 1994), and is presented as a percentage of the maximal output of the TMS stimulator. Conditions NM, E, EE, EW, EA (see table 3.1) were also evaluated when the coil was held at $10 \mathrm{~cm}$ from the head. At this distance, there is no brain activation induced by the magnetic pulse, which falls off with square of the distance from the coil (Griffiths 2008), but only an auditory stimulus due to the TMS clicking sound. 


\begin{tabular}{|l|l|l|l|}
\hline \multicolumn{5}{|l|}{ Table 3.1. Experiment 1: Masking conditions and motor threshold determination } \\
\hline Condition & Masking & Motor threshold & 10 centimeter \\
\hline NM & None & Yes & Yes \\
\hline E & Earplugs & No & Yes \\
\hline EE & Earplugs + earmuff & No & Yes \\
\hline EEF & Earplugs + earmuff + foam & No & No \\
\hline EW & Earplugs + white noise & No & Yes \\
\hline EWF & Earplugs + white noise + foam & No & No \\
\hline EA & Earplugs + adapted noise & Yes & Yes \\
\hline EAF & Earplugs + adapted noise + foam & Yes & No \\
\hline Fa & Foam & Yes & No \\
\hline
\end{tabular}

a) Condition F was only used for evaluating the effect of the layer of foam on the motor threshold.

In experiment 2, we evaluated conditions NM (no masking) and EA (masking with earplugs/adapted noise) in the deaf subject and in a hearing subject (subject 10). The coil was now positioned at four different distances from the head; at $10 \mathrm{~cm}$, at $6 \mathrm{~cm}$ and $2 \mathrm{~cm}$ with Plexiglas between coil and head to mimic bone conduction of sound, and at $0.5 \mathrm{~cm}$ with a layer of foam between coil and head. This resulted in 8 conditions, listed in table 3.2.

\begin{tabular}{|l|l|}
\hline \multicolumn{2}{|l|}{ Table 3.2. Experiment 2: Masking conditions at various distances } \\
\hline Condition & Masking \\
\hline $10 \mathrm{~cm}$ & no masking \\
\hline $10 \mathrm{~cm}$ & earplugs + adapted noise \\
\hline $6 \mathrm{~cm}$ with Plexiglas & no masking \\
\hline $6 \mathrm{~cm}$ with Plexiglas & earplugs + adapted noise \\
\hline $2 \mathrm{~cm}$ with Plexiglas & no masking \\
\hline $2 \mathrm{~cm}$ with Plexiglas & earplugs + adapted noise \\
\hline $0.5 \mathrm{~cm}$ with foam & no masking \\
\hline $0.5 \mathrm{~cm}$ with foam & earplugs + adapted noise \\
\hline
\end{tabular}


We performed additional control measurements in two hearing subjects (subject 11 and 12). In these measurements, we compared (1) external headphones with inserted earphones; (2) the effect of using foam alone; (3) the response to sham stimulation, where we held the coil sideways on top of the head, i.e. tilted by $90^{\circ}$. For measurement 1 we tested all conditions from the original experiment 1, using external headphones as well as inserted earphones. For measurement 2 we tested conditions NM (no masking), F (foam), E and EF (earplugs without and with foam), W and WF (white noise without and with foam), A and AF (adapted noise without and with foam), using both external headphones and inserted earphones. For measurement 3 we tested all conditions from the original experiment 1 using both external headphones and inserted earphones, with the coil tilted sideways.

\section{Masking strategies}

The earplugs (3M, St. Paul, Minnesota, United States) had an SNR (single number rating) of $37 \mathrm{~dB}$ and the earmuff (also from $3 \mathrm{M}$ ) had an SNR of $35 \mathrm{~dB}$. The layer of foam was $0.5 \mathrm{~cm}$ thick, but when placed between coil and head it was pushed in substantially by the coil, with a remaining thickness of approximately $2 \mathrm{~mm}$. To create the adapted noise, we recorded the sound of the coil click and generated noise which contained the same frequency content as the coil click itself. The intensity of the white noise and adapted noise was set for each subject individually, by increasing the volume until the subject reported that he or she could no longer hear the coil click, or until the maximum volume of our equipment $(95 \mathrm{~dB})$ was reached. For the conditions with the coil placed directly on top of the head or with foam between coil and head, all subjects required this maximum volume of $95 \mathrm{~dB}$.

\section{TMS targeting}

Single biphasic TMS pulses, with pulse duration of $400 \mu$ s and a random inter-pulse interval between 2 and 4 seconds, were delivered manually, using a $70 \mathrm{~mm}$ figureof-eight air film coil and a Rapid ${ }^{2}$ stimulator (The Magstim Company Ltd, Whitland, United Kingdom). The coil was placed tangentially over the hot-spot of the abductor digiti minimi muscle (ADM) in the left hemisphere, with the handle pointing backward and laterally at an angle $45^{\circ}$ away from the midline. The maximum stimulator output was 1.5 tesla; stimulation intensity during the masking conditions was always set at $80 \%$ of this output to ensure the same TMS clicking sound intensity for all subjects. For each masking condition we applied 50 TMS pulses. 
Positioning of the coil was achieved using a robot-navigated system (Advanced Neuro Technology, Enschede, Netherlands). A headband carrying four passive reflective markers was fixed to the head of the subject and tracked by a Polaris infrared camera system (Northern Digital Inc., Waterloo, Ontario, Canada). The robot and the tracking system were registered to a common coordinate system using a calibration procedure. The robot-guided TMS coil was added to the coordinate system by registration of three reference positions on the coil using a tracking pointer. A standard 1.5 tesla MRI scan was used to create a head model; this model was then registered to the subject's head and the coordinate system by collecting three landmarks and $\sim 300$ additional points with a tracking pointer.

\section{Electromyography}

To determine the motor threshold, surface electrodes were placed in a belly-tendon montage over the right ADM muscle. The ground electrode was placed on the dorsal side of the wrist. We recorded the EMG using an additional amplifier (TMSi, Oldenzaal, Netherlands) connected to the EEG amplifier, ensuring synchronized measurements. EMG was sampled at $2048 \mathrm{~Hz}$ and low-pass filtered with an antialiasing filter with a cut-off frequency of $550 \mathrm{~Hz}$.

\section{EEG recording and analysis}

The EEG was recorded continuously during TMS using a 64-channel DC amplifier (TMSi, Oldenzaal, Netherlands) and a TMS-compatible 64-electrode cap (ANT, Enschede, Netherlands). Electrode impedances were kept below $5 \mathrm{kOhm}$. The EEGsignals were low-pass filtered using an anti-aliasing filter with a cut-off frequency of $550 \mathrm{~Hz}$ and sampled at $2048 \mathrm{~Hz}$. The ground electrode was placed between electrode positions $\mathrm{Fz}$ and Fpz. We used a common average reference for the recordings. A single TMS pulse produced a stimulation artefact of 1-2 $\mathrm{mV}$, lasting approximately 5 samples $(2.5 \mathrm{~ms})$. For analysis, the signal was re-referenced to the average of the left and right mastoid bone. Trials were defined from one second before to one second after every TMS pulse, resulting in 50 trials of two seconds for each masking condition per subject. Trials with eye-blinks were automatically rejected using a fixed threshold of $150 \mu \mathrm{V}$ for electrode channel $\mathrm{Cz}$, which resulted in at least 45 accepted trials for all subjects. In the remaining trials, we removed the offset by subtracting the baseline. Then, we replaced the samples of the TMS artefact with a linear interpolation between six samples preceding and fourteen samples following the start of the TMS artefact. After this, trials were band-pass filtered 
between 1 and $50 \mathrm{~Hz}$ using a fourth order Butterworth filter. The filtered trials were then averaged to obtain the TEP, and the response measured at electrode $\mathrm{Cz}$ was selected for further analysis. After manually selecting a specific latency window for each subject, condition and peak, based on visual inspection of the TEP, we determined the amplitude of the N100 and P180 component automatically and compared the peak-to-peak amplitude between masking conditions for all subjects, normalized with respect to the no masking condition. When no clear peaks or multiple peaks were visible, we used the latencies of previous selected peaks in that subject to select a data point showing the highest amplitude within that latency range. Because the P50 could not be identified in all subjects, we evaluated the presence of this component before and after masking through visual inspection.

\section{Statistical analysis}

The motor thresholds and determined peak-to-peak amplitude of the N100-P180 complex for each masking condition were compared with the no masking condition for each subject. This was also done for the foam conditions with respect to the nofoam conditions. A paired $t$-test was used to test for statistical significance. Differences with $p<0.05$ were considered significant.

\section{Results}

There were no adverse events during the TMS measurements. All subjects showed a characteristic TEP response, although there was a variation in amplitude, caused by the difference in each subject's MT and the stimulation intensity of $80 \%$ that was used for the experimental conditions. In all subjects, the N100-P180 complex reduced in amplitude when masking was applied. One of the other components of the AEP, the P50, could be identified in 4 subjects, and was completely suppressed in 3 of these subjects in the adapted noise (+ foam) masking condition (EAF). Figure 3.1, presenting the TEP in a single subject, shows the reduction in amplitude of the N100-P180 complex, and the disappearance of the P50 when the earmuff (EEF) was replaced by headphones playing adapted noise (EAF). 


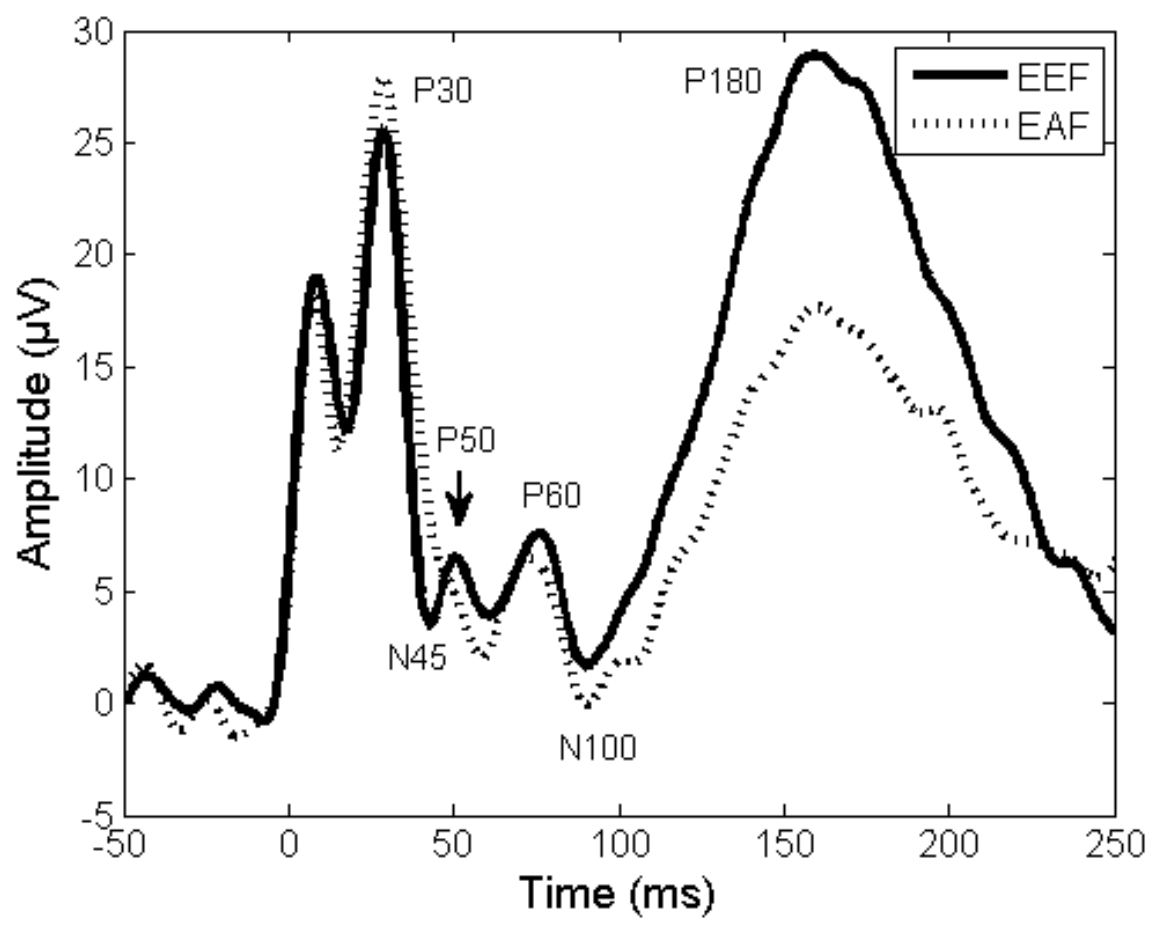

Figure 3.1. TEP response at electrode $\mathrm{Cz}$ in a single subject in two different masking conditions. When an earmuff with a layer of foam is used, there is a P50 visible (indicated with the arrow), which is part of the AEP. When the earmuff is replaced with earphones playing adapted noise, the P50 disappears. EEF: masking condition with earplugs/earmuff/foam; EAF: masking condition with earplugs/adapted noise/foam. 
The effect of different masking techniques on the normalized N100-P180 amplitude when the coil is held at $10 \mathrm{~cm}$ from the head is shown in figure 3.2 (upper panel). Earplugs alone, or in combination with an earmuff, reduced the amplitude significantly. The N100-P180 was removed completely in all subjects when either white or adapted noise was used, demonstrated by a response amplitude that resembled baseline activity.
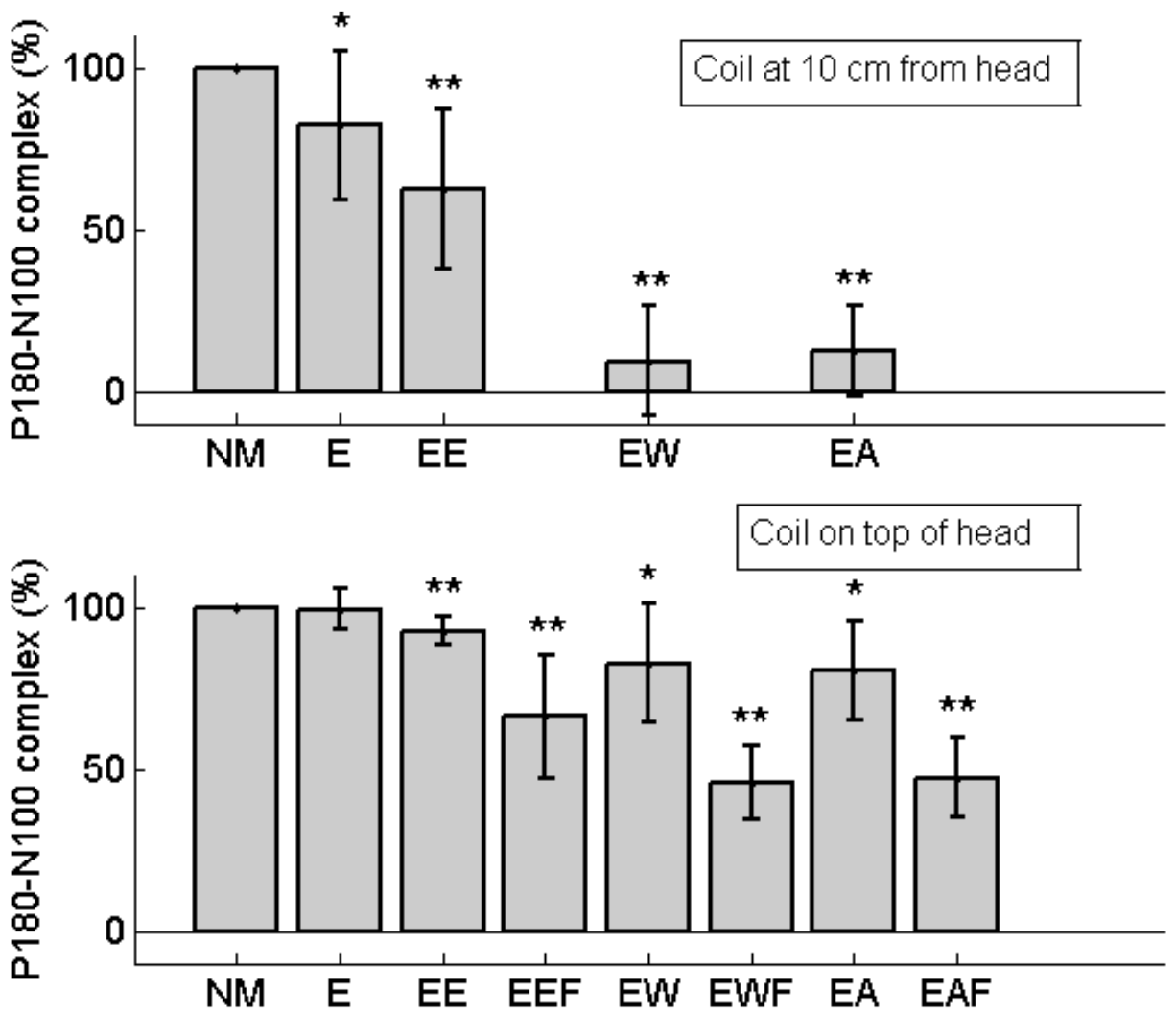

Figure 3.2. Amplitude of the 100-180 complex when different masking conditions are used, average of 9 hearing subjects. All amplitudes are shown relative to the baseline condition (NM). Results are shown for when the coil was held at $10 \mathrm{~cm}$ from the head (top figure) and when the coil was held on top of the head (bottom figure). Errorbars represent the standard deviation. $* p<0.05$ and $* * p<0.001$ when compared to the baseline condition (NM). Abbreviations indicating the different masking conditions can be found in table 3.1. 
Reducing the N100-P180 complex proved to be much more difficult when the coil was held on top of the head (figure 3.2, lower panel). Earplugs alone caused no significant reduction in N100-P180 amplitude. The earmuff did reduce the amplitude significantly, but the amount of reduction was small. Exposing the subjects to either white or adapted noise also caused a significant reduction in N100-P180, but the largest reduction was achieved when adding a layer of foam between coil and head ( $\mathrm{p}<0.01$ for all foam conditions compared to no foam conditions: EEF/EE, EWF/EW and EAF/EA). However, we were not able to completely suppress the N100-P180 complex when the coil was held on top of the head. The intensity of the masking sound (white or adapted noise) was $95 \mathrm{~dB}$ for all subjects when the coil was held on top of the head, but four of the twelve hearing subjects (including subject 10, 11 and 12) reported still hearing the clicking sound. With the coil in the $10 \mathrm{~cm}$ position, a lower intensity of the masking sound sufficed; none of the subjects still heard the clicking sound.

In the nine hearing subjects, the motor threshold significantly increased when a layer of foam was placed between coil and head (NM 59\% vs. foam $70 \%, p<0.001$, and EA $57 \%$ vs. EAF $70 \%, p<0.001$ ), which is most likely caused by the small increase in distance between coil and the target brain area. Using adapted noise did not change the motor threshold (NM 59\% vs. EA 57\%, $p=0.08$ ).

In figure 3.3, we show the results from experiment 2. The hearing subject showed a clear AEP, both when the coil was placed at $10 \mathrm{~cm}$ and $6 \mathrm{~cm}$ from the head. The AEP components disappeared after masking was applied. The deaf subject did not show an AEP at these two distances. When the coil was kept closer to the head, the deaf subject showed a TEP with characteristic components at 30, 45, 60, 100 and $180 \mathrm{~ms}$. The TEP in the hearing subject did respond to the applied masking condition: the N100-P180 amplitude decreased, and the P60 was reduced after masking. Visually, it seems that the amplitude of the P180 is reduced more easily than the N100 amplitude. The P180 increased as the coil was positioned closer to the head, ranging from $17 \mu \mathrm{V}$ at $10 \mathrm{~cm}$ to $60 \mu \mathrm{V}$ at $0.5 \mathrm{~cm}$. For the $0.5 \mathrm{~cm}$ condition, there appears to be a small modulation in the deaf subject, comparable to the change observed in the hearing subject. 

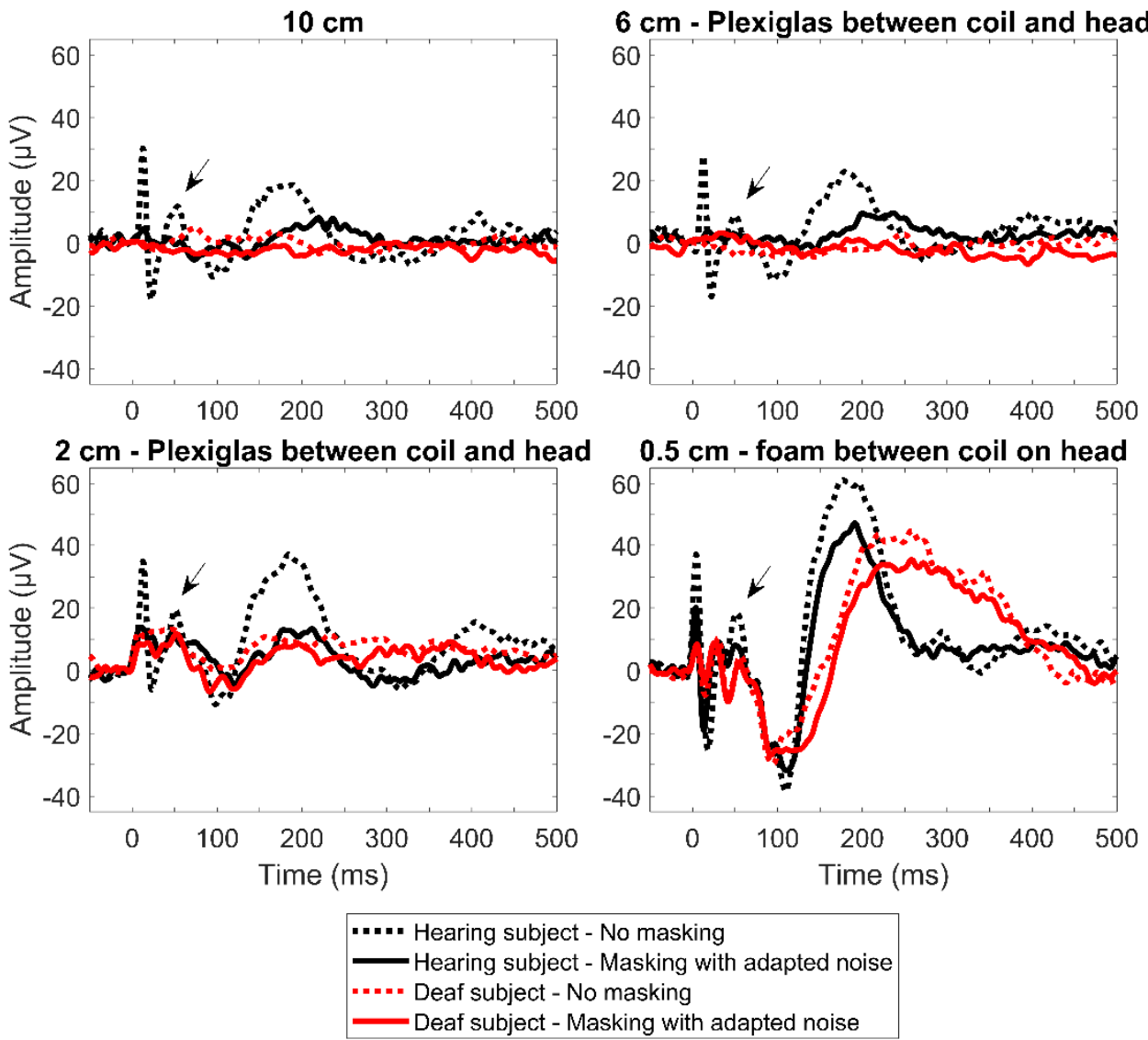

Figure 3.3. TMS evoked potential in a hearing subject and a deaf subject for experiment 2 . The deaf subject shows no response to only auditory stimuli (top figures, red lines), but when the coil is held close to the head (bottom figures, red lines), there is a TEP visible with characteristic components (P30-N45-P60-N100-P180). The hearing subject (black lines) shows an AEP when the coil is held at $10 \mathrm{~cm}$ and $6 \mathrm{~cm}$ from the head, which disappears when masking is applied. The P60, marked with arrows, reduces in amplitude when masking is applied. The amplitude of the N100-P180 complex reduces as well after masking. Arrows indicate the P60 in the hearing subject in the no masking condition. Note, that the amplitude of the P180 in the hearing subject after masking is of similar amplitude as the P180 in the deaf subject (bottom right panel). 
When we applied sham stimulation as a control measurement in the two hearing subjects, we found a P50-N100-P180 (see figure 3.4). These AEP components were reduced by masking. When we applied white or adapted noise during sham stimulation, the response was completely suppressed, even without using foam, as can be seen in figure 3.4. We found no differences in the response using external headphones or inserted headphones. Foam alone did reduce the N100-P180 amplitude, but not as much as using white or adapted noise alone.

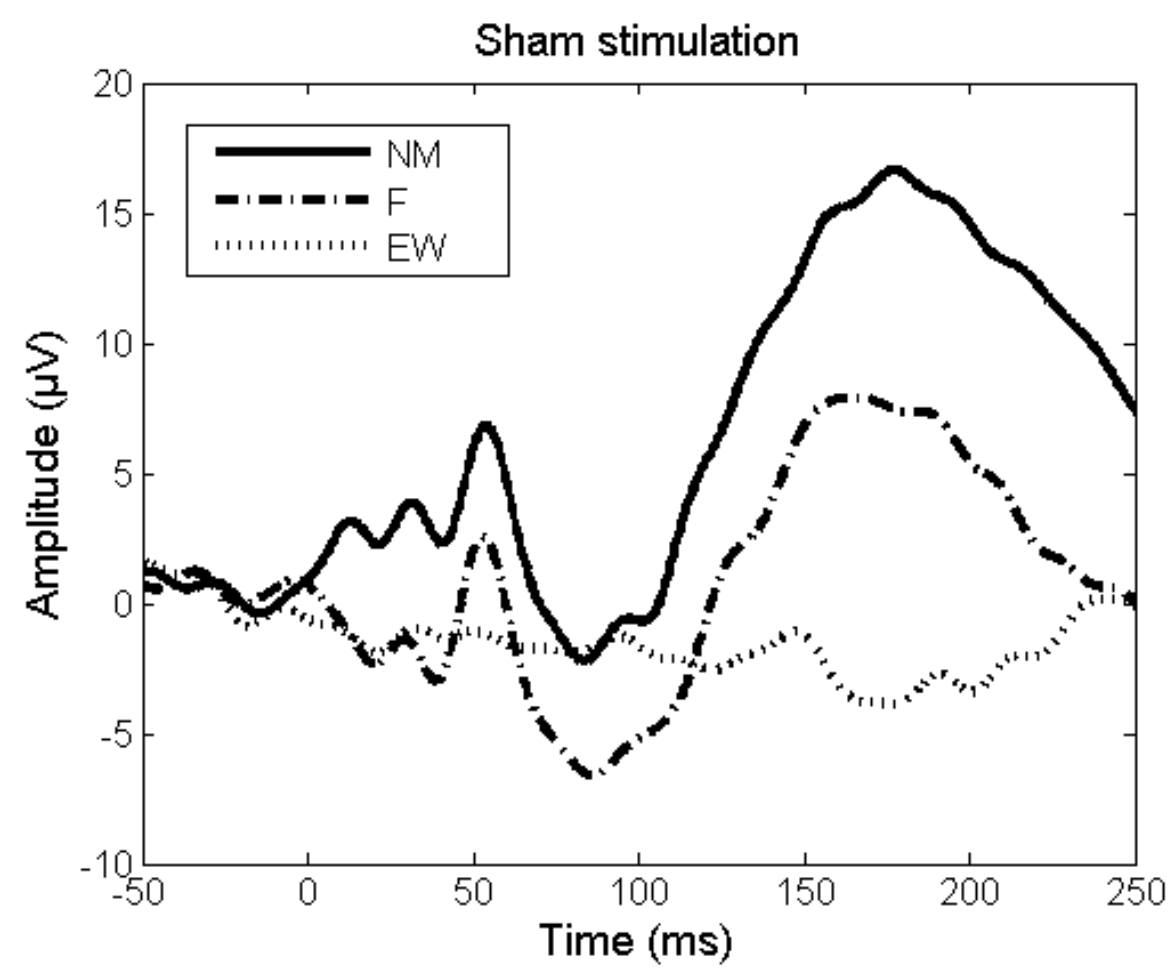

Figure 3.4. Auditory evoked responses in the control measurements (coil tilted sideways on top of the head) in a hearing subject. Foam alone causes a small reduction in N100-P180 amplitude, comparable to using earplugs alone. Adding white noise (so without foam) completely suppresses the AEP, including the P50. 


\section{Discussion}

The physiological processes responsible for the different components of the TEP are still unknown (Ilmoniemi and Kičić 2010). However, it is very likely that the TEP is, at least partly, influenced by the clicking sound of the TMS coil (Nikouline et al. 1999). Therefore, an adequate masking technique may reduce the contribution of the AEP to the TMS evoked response. In this study, we compared different types of masking used in TMS-EEG experiments. Furthermore, we performed measurements in a deaf subject to obtain a TEP that is not at all influenced by auditory stimuli.

The AEP that is evoked by the TMS clicking sound can be identified by the N100P180 complex, but also, in some subjects, by the presence of the P50 in the TEP. We show that white or adapted noise is able to completely remove the AEP caused by the TMS sound, when the coil is held at $10 \mathrm{~cm}$ from the head. At this distance the TMS magnetic pulse does not induce any activation in the brain, so the AEP is the only response visible in the EEG.

Using white or adapted noise also removed the AEP when the coil was positioned at $6 \mathrm{~cm}$ from the head, with a piece of Plexiglas between coil and head to mimic bone conduction of sound. This condition has been used before to prove that playing noise removes all auditory evoked activity from the response (Massimini et al. 2005). In another study, it was shown that no auditory evoked potential was measured when playing white noise and positioning the coil $2 \mathrm{~cm}$ from the head (Paus et al. 2001), but this study did not explore the bone conduction of sound. In our measurements, the auditory response was smaller when we removed the piece of Plexiglas (results not shown), confirming that bone conduction did increase the amplitude of the AEP. As the coil was moved closer to the head $(10-6-2-0.5 \mathrm{~cm})$, the intensity of the clicking sound may have also increased, adding to the gradual increase in amplitude of the N100-P180.

When the coil was touching the head, the N100-P180 amplitude is also modulated by the use of various masking methods, but it always remains present in the evoked response. This suggests that these components are also partly induced by the magnetic field of the pulse. Using white or adapted noise decreases the N100-P180 considerably, but adding a layer of foam between coil and head resulted in the largest attenuation of the AEP, reducing to half of its amplitude compared to noise alone. Using this additional layer of foam is therefore essential in minimizing the auditory 
components of the AEP, most likely because it minimizes bone-conduction. Foam alone did not reduce the AEP as much as listening to white or adapted noise alone, so the strength of successful masking lies in combining both foam and noise. It has to be noted that with foam the motor threshold increases significantly, which is most likely due to the increased distance between the coil and targeted brain area. This agrees with a previous report of an increased motor threshold when an EEG cap was placed between coil and head compared to measurements without the cap. This increase was also caused by an increase in the distance between coil and head (Julkunen et al. 2009). In addition, the focality of stimulation may also change for different stimulation distances.

The amplitude of the different TEP components may be influenced by numerous factors, such as a decrease in N100 amplitude when preparing a voluntary movement (Kičić et al. 2008; Nikulin et al. 2003). However, it is possible that observed variations in $\mathrm{N} 100$ or $\mathrm{P} 180$ partly reflect variations in auditory processing in addition to a TMS effect, and therefore N100 and P180 (and perhaps even P60) modulation should be used carefully as an outcome measure when no masking or a non-sufficient masking method is used. For example, in one of our subjects parts of the AEP remained in the TEP after masking, illustrated by the remaining presence of a P50 component. This may have been the case as well for other subjects, as the P50 latency may overlap with the P60 of the TEP. Figure 3.3 shows that the P60 reduces in amplitude after masking is applied, suggesting a hidden P50 auditory component. As a matter of fact, in five hearing subjects we could not even identify the P50 before masking. Still, the P50 should not be visible after successful masking, and its presence after masking indicates that the AEP is not sufficiently reduced.

Some of our subjects reported still hearing the TMS clicking sound with a headphone playing white or adapted noise, which may have been caused by the fact that subjects were instructed to listen closely to the TMS sound while adapting the noise intensity. This possibly led them to also focus more on the TMS sound during the rest of the experiment, thereby increasing the amplitude of the AEP (Schiff et al. 2008). The presence of the AEP during protocols in which subjects focus on a specific (nonauditory) task may therefore be less prominent. On the other hand, it could also be that the subjects found it difficult to differentiate between hearing and feeling the TMS pulse: we were unable to assess this possible difference. 
We performed a TMS-EEG experiment in a deaf subject to further differentiate between the TEP and AEP, as our measurements in the hearing subjects could not fully reveal what the exact contribution of the AEP is to the N100-P180 complex. The results from this additional experiment showed that the N100 and P180 were also present in the deaf subject, confirming that these components in hearing subjects are indeed largely induced by the magnetic pulse. There appears to be a small modulation in the deaf subject as well, comparable to the change observed in the hearing subject. This change may result from normal variation or noise between different measurements, and/or a minimal hearing ability in the deaf subject although she showed no response on the audiogram nor reported any awareness of the sound. In either case, the presence of this large N100-P180 component in the deaf subject strongly suggests a non-auditory contribution. In the hearing subject, the other characteristic TEP components (P30-N45) were not modulated by masking, and therefore these peaks are certainly solely TMS induced. Although this was a single case comparison, it proves that it is indeed highly unlikely that the N100 and P180 are only induced by auditory effects.

In the control measurement using sham stimulation with the coil sideways touching the head, we found no early TEP components, but only a P50, N100 and P180. When we applied masking, this response was completely suppressed. The sham stimulation aimed at mimicking the clicking sound with bone conduction. However, no foam was required in this sham condition to eliminate the response (see figure 3.4), suggesting that bone conduction did not play an important part in the auditory processing. This corresponds to the results of the Plexiglas condition at $6 \mathrm{~cm}$, where the response was also already eliminated with only noise masking, so without applying foam. Conversely, when the coil is held in a normal position (directly on top of the head), foam does further reduce the amplitude of the N100 and P180 components. Because foam increases the distance between coil and head, the amplitude reduction using foam may be partly due to a small decrease in intensity of the magnetic field. Another possible explanation for this discrepancy could be that the Plexiglas condition, as well as the sham condition, does not mimic bone conduction that well after all. However, the response did decrease in amplitude when we removed the Plexiglas layer, suggesting that there was indeed bone conduction, although possibly less pronounced than with the coil touching the head. With the Plexiglas layer, the intensity of the masking sound was probably high enough to mask this less pronounced bone conduction component so no foam was needed. 
The noise masking might have been more efficient with the use of inserted headphones, as used by previous authors (Fuggetta et al. 2005; Massimini et al. 2005; Paus et al. 2001; Veniero et al. 2010; Werf and Paus 2006). Therefore, we compared external and inserted headphones in two subjects. We found no differences in the evoked potentials between these two conditions, although one of the subjects reported that the inserted earphones provided better masking. The intensity of the masking sound in the external headphone and inserted headphone was the same, which may be the most important feature to successfully mask the TMS clicking sound. A disadvantage of regular headphones is that they may interfere with the coil positioning when stimulating the temporal regions.

There are limitations in our study. We applied TMS at the same stimulus intensity for all subjects, independent of their motor threshold. This was done to evaluate the performance of the masking conditions in a more standardized way. However, for every individual subject this stimulation intensity related differently to the motor threshold, which could be higher or lower than the used settings. This introduces variations in TEP amplitudes, including the TMS induced part of the N100-P180 complex.

We did not evaluate the amplitude change of the N100 and P180 separately, but it appeared that the N100 was less influenced by masking than the P180. This could indicate that the N100 is largely induced by TMS activation, meaning that the auditory components only account for a small part of the N100, making the N100 a more stable output measure than the P180 when evaluating TEP modulation.

With a relatively small number of averaged responses we obtained well-defined TEPs, which showed large differences in N100-P180 amplitude. However, using more trials may further improve the signal-to-noise ratio, which could be necessary to evaluate very small changes in the TEP between groups or experimental conditions.

As Nikouline et al suggested (Nikouline et al. 1999), there could be some sensory effects caused by activation of the skin by the magnetic pulse, so called somatosensory evoked potentials (SSEP). When the coil is held directly on top of the head, there may still be a SSEP present, adding to the TEP in both the hearing 
subjects and the deaf subject. Our measurements did not enable us to differentiate between the SSEP and magnetic activation of the cortex.

To summarize, masking strongly diminishes the AEP components in the TMS evoked responses. Using white or adapted noise combined with a layer of foam between coil and head results in the largest reduction of these auditory responses. Although the TMS clicking sound contributes to the N100-P180 amplitude, our results confirm that this response is for the most part induced by the magnetic pulse itself.

\section{Acknowledgements}

The authors wish to thank Benjamin de Jonge, MSc, for his assistance during the measurements. 


\section{References}

Barker AT, Jalinous R, Freeston IL. "Non-invasive magnetic stimulation of human motor cortex". Lancet 1985; 1: 1106-1107

Bikmullina R, Kičić D, Carlson S, Nikulin VV. "Electrophysiological correlates of shortlatency afferent inhibition: a combined EEG and TMS study". Exp Brain Res 2009; 194: 517-526

Bonato C, Miniussi C, Rossini PM. "Transcranial magnetic stimulation and cortical evoked potentials: a TMS/EEG co-registration study". Clin Neurophysiol 2006; 117: 16991707

Casali AG, Casarotto S, Rosanova M, Mariotti M, Massimini M. "General indices to characterize the electrical response of the cerebral cortex to TMS". Neuroimage 2010; 49: 1459-1468

Casarotto S, Lauro LJR, Bellina V, Casali AG, Rosanova M, Pigorini A et al. "EEG responses to TMS are sensitive to changes in the perturbation parameters and repeatable over time". PLoS One 2010; 5: e10281

Chen R, Cros D, Curra A, Lazzaro VD, Lefaucheur J-P, Magistris MR et al. "The clinical diagnostic utility of transcranial magnetic stimulation: report of an IFCN committee". Clin Neurophysiol 2008; 119: 504-532

Esser SK, Huber R, Massimini M, Peterson MJ, Ferrarelli F, Tononi G. "A direct demonstration of cortical LTP in humans: a combined TMS/EEG study". Brain Res Bull 2006; 69: 86-94

Ferrarelli F, Massimini M, Sarasso S, Casali A, Riedner BA, Angelini G et al. "Breakdown in cortical effective connectivity during midazolam-induced loss of consciousness". Proc Natl Acad Sci U S A 2010; 107: 2681-2686

Ferreri F, Pasqualetti P, Määttä S, Ponzo D, Ferrarelli F, Tononi G et al. "Human brain connectivity during single and paired pulse transcranial magnetic stimulation". Neuroimage 2011; 54: 90-102

Fuggetta G, Fiaschi A, Manganotti P. "Modulation of cortical oscillatory activities induced by varying single-pulse transcranial magnetic stimulation intensity over the left primary motor area: a combined EEG and TMS study". Neuroimage 2005; 27: 896908

Griffiths DJ (2008) Introduction to Electrodynamics. Third edn. Pearson Benjamin Cummings, San Fransisco

Hamidi M, Slagter HA, Tononi G, Postle BR. "Brain responses evoked by high-frequency repetitive transcranial magnetic stimulation: an event-related potential study". Brain Stimul 2010; 3: 2-14

Ilmoniemi RJ, Kičić D. "Methodology for combined TMS and EEG". Brain Topogr 2010; 22: $233-248$ 
Julkunen P, Jauhiainen AM, Westerén-Punnonen S, Pirinen E, Soininen H, Könönen M et al. "Navigated TMS combined with EEG in mild cognitive impairment and Alzheimer's disease: a pilot study". J Neurosci Methods 2008; 172: 270-276

Julkunen P, Säisänen L, Sarasti M, Könönen M. "Effect of electrode cap on measured cortical motor threshold". J Neurosci Methods 2009; 176: 225-229

Kähkönen S, Kesäniemi M, Nikouline VV, Karhu J, Ollikainen M, Holi M, Ilmoniemi RJ. "Ethanol modulates cortical activity: direct evidence with combined TMS and EEG". Neuroimage 2001; 14: 322-328

Kähkönen S, Komssi S, Wilenius J, Ilmoniemi RJ. "Prefrontal TMS produces smaller EEG responses than motor-cortex TMS: implications for rTMS treatment in depression". Psychopharmacology (Berl) 2005a; 181: 16-20

Kähkönen S, Komssi S, Wilenius J, Ilmoniemi RJ. "Prefrontal transcranial magnetic stimulation produces intensity-dependent EEG responses in humans". Neuroimage 2005b; 24: 955-960

Kičić D, Lioumis P, Ilmoniemi RJ, Nikulin VV. "Bilateral changes in excitability of sensorimotor cortices during unilateral movement: combined electroencephalographic and transcranial magnetic stimulation study". Neuroscience 2008; 152: 1119-1129

Komssi S, Kähkönen S, Ilmoniemi RJ. "The effect of stimulus intensity on brain responses evoked by transcranial magnetic stimulation". Hum Brain Mapp 2004; 21: 154-164

Levit-Binnun N, Litvak V, Pratt H, Moses E, Zaroor M, Peled A. "Differences in TMSevoked responses between schizophrenia patients and healthy controls can be observed without a dedicated EEG system". Clin Neurophysiol 2010; 121: 332-339

Lioumis P, Kičić D, Savolainen P, Mäkelä JP, Kähkönen S. "Reproducibility of TMS-Evoked EEG responses". Hum Brain Mapp 2009; 30: 1387-1396

Mäki H, Ilmoniemi RJ. "The relationship between peripheral and early cortical activation induced by transcranial magnetic stimulation". Neurosci Lett 2010; 478: 24-28

Massimini M, Ferrarelli F, Huber R, Esser SK, Singh H, Tononi G. "Breakdown of cortical effective connectivity during sleep". Science 2005; 309: 2228-2232

Massimini M, Ferrarelli F, Murphy M, Huber R, Riedner B, Casarotto S, Tononi G. "Cortical reactivity and effective connectivity during REM sleep in humans". Cogn Neurosci 2010; 1: 176-183

Miniussi C, Thut G. "Combining TMS and EEG Offers New Prospects in Cognitive Neuroscience". Brain Topogr 2010; 22: 249-256

Nikouline V, Ruohonen J, Ilmoniemi RJ. "The role of the coil click in TMS assessed with simultaneous EEG". Clin Neurophysiol 1999; 110: 1325-1328

Nikulin VV, Kičić D, Kähkönen S, Ilmoniemi RJ. "Modulation of electroencephalographic responses to transcranial magnetic stimulation: evidence for changes in cortical excitability related to movement". Eur J Neurosci 2003; 18: 1206-1212 
Padberg F, George MS. "Repetitive transcranial magnetic stimulation of the prefrontal cortex in depression". Exp Neurol 2009; 219: 2-13

Paus T, Sipila PK, Strafella AP. "Synchronization of neuronal activity in the human primary motor cortex by transcranial magnetic stimulation: an EEG study". J Neurophysiol 2001; 86: 1983-1990

Picton TW, Hillyard SA, Krausz HI, Galambos R. "Human auditory evoked potentials. I. Evaluation of components". Electroencephalogr Clin Neurophysiol 1974; 36: 179190

Rosanova M, Casali A, Bellina V, Resta F, Mariotti M, Massimini M. "Natural frequencies of human corticothalamic circuits". J Neurosci 2009; 29: 7679-7685

Rossi S, Hallett M, Rossini PM, Pascual-Leone A, of TMS Consensus Group TS. "Safety, ethical considerations, and application guidelines for the use of transcranial magnetic stimulation in clinical practice and research". Clin Neurophysiol 2009; 120: 2008-2039

Rossini PM, Barker AT, Berardelli A, Caramia MD, Caruso G, Cracco RQ et al. "Noninvasive electrical and magnetic stimulation of the brain, spinal cord and roots: basic principles and procedures for routine clinical application. Report of an IFCN committee". Electroencephalogr Clin Neurophysiol 1994; 91: 79-92

Schiff S, Valenti P, Andrea P, Lot M, Bisiacchi P, Gatta A, Amodio P. "The effect of aging on auditory components of event-related brain potentials". Clin Neurophysiol 2008; 119: 1795-1802

ter Braack EM, de Jonge B, van Putten MJ. "Reduction of TMS Induced Artifacts in EEG Using Principal Component Analysis". IEEE Trans Neural Syst Rehabil Eng 2013; 21: 376-382

Tiitinen H, Virtanen J, Ilmoniemi RJ, Kamppuri J, Ollikainen M, Ruohonen J, Näätänen R. "Separation of contamination caused by coil clicks from responses elicited by transcranial magnetic stimulation". Clin Neurophysiol 1999; 110: 982-985

Veniero D, Maioli C, Miniussi C. "Potentiation of short-latency cortical responses by highfrequency repetitive transcranial magnetic stimulation". J Neurophysiol 2010; 104: 1578-1588

Werf YDVD, Paus T. "The neural response to transcranial magnetic stimulation of the human motor cortex. I. Intracortical and cortico-cortical contributions". Exp Brain Res 2006; 175: 231-245 


\section{Chapter 4}

\section{Resting motor threshold and TMS-EMG-EEG evoked responses during daytime}

Esther M. ter Braack, Annika A. de Goede, Michel J.A.M. van Putten Submitted in revised form (Brain Topography) 


\section{Abstract}

Background: Humans show a variation in physiological processes during the day. To reliably assess (changes in) cortical excitability with transcranial magnetic stimulation (TMS), it is relevant to know the natural variation in TMS readouts during the day. In case of significant daytime variations, this should be taken into account when scheduling (follow-up) measurements.

Objective: To study the influence of the time of day on the resting motor threshold (RMT), motor evoked potential (MEP and TMS evoked potential (TEP) in healthy controls.

Methods: TMS-EMG-EEG was recorded in 16 healthy subjects. At both motor cortices, we administered 75 pulses at an intensity of $110 \%$ RMT. Subjects were stimulated during five sessions in one day (8:00AM, 10:30AM, 1:00PM, 3:30PM and 6:00PM) while keeping the stimulation intensity constant. We compared the TEP waveforms between the five sessions with a cluster-based permutation analysis, and the RMT and MEP amplitude with rmANOVA.

Results: In general there were no significant differences between the five sessions in the RMT, MEP amplitude or TEP. Only for the left side, N100 amplitude was larger at 3:30PM than 10:30AM. The standard deviation of the P30 and N100 amplitude was significantly higher between subjects within one session than within single subjects during the day.

Conclusion: The TEP is highly reproducible during the day, with a low intraindividual variation compared to the inter-individual variation. In addition, we found no significant variation of the motor threshold and MEP amplitude between multiple sessions on one day. 


\section{Introduction}

Humans, and animals and plants as well, show a variation in physiological processes during the day. This circadian rhythm is regulated by our biological clock, resulting in diurnal fluctuations in for instance hormone secretion, blood pressure, but also alertness (Nobelprize.org). Some epilepsies show a relationship with sleep stages or the transition between sleep and wakefulness, of which juvenile myoclonic epilepsy (JME) is the most typical example where seizures occur predominantly after awakening in the morning. Besides the interaction between sleep and epilepsy, the time of day is also correlated with seizure occurrence in some focal epilepsy types (Hofstra and de Weerd 2009; Mirzoev et al. 2012; van Campen et al. 2015). This diurnal distribution of seizures is mainly evident for temporal lobe epilepsy, with a peak in seizure occurrence in the late afternoon (Durazzo et al. 2008; Hofstra et al. 2009; Pavlova et al. 2004).

Epilepsy can be characterized as a disease resulting from an imbalance between cortical excitation and inhibition. Transcranial magnetic stimulation (TMS) is a wellestablished technique to non-invasively activate brain areas (Barker et al. 1985), and is a promising method to assess cortical excitability, which we here define as the strength of the response of cortical neurons to an external input. The resting motor threshold (RMT) or the MEP amplitude following a neuromodulatory paradigm (paired-pulse TMS) can be used as readouts of cortical excitability. Combining TMS with EEG has also become available (Ilmoniemi and Kičić 2010; Miniussi and Thut 2010), opening novel possibilities to study cortical excitability. A single TMS pulse induces a response in the EEG, and after averaging over multiple pulses, the TMS evoked potential (TEP) is obtained. TMS-EEG could provide a more direct measure of cortical excitability than measuring the MEP (Bonato et al. 2006; Ferreri et al. 2011; Ilmoniemi and Kičić 2010), as it is not influenced by the excitability of corticospinal and spinal neurons. The TEP has been shown to change after administration of GABA-ergic drugs as well as anti-epileptic drugs (Premoli et al. 2017; Premoli et al. 2014).

TMS is considered to be a candidate tool for a new biomarker in epilepsy (Bauer et al. 2014; Chen et al. 2008; Engel 2008; Kimiskidis 2016; Manganotti and del Felice 2013). To reliably assess (changes in) cortical excitability, it is relevant to know the natural variation in TMS readouts during the day. When there is a significant effect of time-of-day, scheduling follow-up measurements should be performed at 
approximately the same time in the morning or afternoon. Previous studies have shown that the RMT and MEP remain constant during the day (Doeltgen and Ridding 2010; Koski et al. 2005; Lang et al. 2011; Strutton et al. 2003). It is known that the TEP changes when different brain areas are stimulated, and also when the stimulation intensity or stimulation angle is varied (Casarotto et al. 2010). While the TEP after stimulating the motor cortex shows high repeatability comparing the first and last part of a TMS-EEG session (Casarotto et al. 2010; Kerwin et al. 2018) and high reproducibility after one week when stimulating parameters are kept constant (Casarotto et al. 2010; Lioumis et al. 2009), diurnal variations of the TEP have not been systematically studied.

The variation of one component of the TEP during daytime has been studied in healthy subjects as a part of larger experimental protocols focusing on the effects of sleep deprivation. The P30 of the TEP did not differ between 9AM and 3PM, but was significantly higher at 9PM compared to 9AM in five out of six subjects (Huber et al. 2012). In a subsequent study by the same research group, the amplitude and slope of the P30 was significantly lower at 9PM compared to 5PM, but no difference was found between 11AM and 5PM (Ly et al. 2016).

The variation of the TEP using multiple measurements during daytime has not been reported before. In this study we investigated the daytime variation of the RMT, MEP amplitude and the TEP after motor cortex stimulation by measuring these responses at 2.5 hour intervals between $8 \mathrm{AM}$ and $6 \mathrm{PM}$ in a group of healthy volunteers.

\section{Material and methods}

The experimental protocol was approved by the local ethics committee (Medisch Spectrum Twente, Enschede, the Netherlands) and was in accordance with the declaration of Helsinki and the guidelines for the use of TMS in clinical practice and research (Rossi et al. 2009). All subjects gave written informed consent.

\section{Subjects}

Nineteen healthy subjects participated in this study. One of these nineteen subjects had a syncope at the start of the first TMS session and was excluded from the study. The remaining eighteen subjects tolerated the TMS protocol well. Part of the data from these eighteen subjects was previously presented, for a different objective (ter 
Braack et al. 2013; ter Braack et al. 2016). Another two subjects were excluded from the analysis, since one subject had a missing session for both targets, and one subject had two missing sessions for one target, both due to technical problems with the navigation system. Sixteen subjects (11 males, mean age 28 years, all right-handed) were therefore included in the analysis.

\section{Experimental protocol}

Subjects were seated in a chair, with their hands pronated in a relaxed position. They kept their eyes open, focusing on a marked point on the wall. Subjects were asked to refrain from alcohol two days and from caffeinated drinks twelve hours prior to measurements. Subjects were only measured if they had a good night of sleep. Each subject underwent one day of measurements, divided in 5 sessions. The sessions took place at 8:00AM, 10:30AM, 1:00PM, 3:30PM and 6:00PM. In three subjects we repeated the first session a week later to confirm previous findings of a good reproducibility (Lioumis et al. 2009).

\section{Stimulation}

Single biphasic TMS pulses, with pulse duration of $400 \mu$ s and inter-pulse interval of $\sim 4$ seconds, were delivered manually using a $70 \mathrm{~mm}$ figure-of-eight air film coil and a Magstim Rapid ${ }^{2}$ stimulator (The Magstim Company Ltd, Whitland, United Kingdom). The maximum stimulator output was 1.5 tesla. The coil was placed tangentially over the hot-spot of the abductor digiti minimi muscle (ADM) in the left and right hemisphere. The handle was pointing backwards and laterally at a $45^{\circ}$ angle away from the midline. At both targets we applied 75 TMS pulses at a stimulation intensity of $110 \%$ of the RMT of the ADM hotspot. This stimulation intensity was kept constant during the day. The motor threshold was defined as the lowest stimulus intensity that produced at least five MEPs of at least $50 \mu \mathrm{V}$ out of 10 consecutive stimuli (Rossini et al. 1994). In four of the sixteen subjects, a TMS intensity of $110 \%$ RMT could not be given due to a too high threshold in session 1. In those subjects, the TMS intensity during the protocol was set to the maximum output of the stimulator (1.5 tesla), corresponding to $100-108 \%$ RMT. During TMS-EEG, all subjects wore protective earplugs, and noise created from the coil click was played through headphones at $95 \mathrm{~dB}$ to mask the sound of the TMS pulses (ter Braack et al. 2015). In addition, a thin layer of foam was placed between the coil and head of the subject to minimize bone conduction. 


\section{TMS targeting}

Positioning of the coil was achieved using a robot-navigated system (Smartmove, ANT Neuro, Enschede, Netherlands), with an accuracy of $1 \mathrm{~mm}$ in every direction. A headband carrying four passive reflective markers was fixed to the head of the subject and tracked by a Polaris infrared camera system (Northern Digital Inc., Waterloo, Ontario, Canada). The robot and the tracking system were registered to a common coordinate system using a calibration procedure. The robot-guided TMS coil was added to the coordinate system by registration of three reference positions on the coil using a tracking pointer. In all subjects, a 1.5 tesla MRI scan of the head was available. The MRI scan was used to create a subject-specific head model; this model was then registered to the subject's head and the coordinate system by collecting three landmarks and 300 additional points on the scalp with a tracking pointer.

\section{EEG and EMG recording during TMS}

The EEG was recorded continuously during TMS using a DC amplifier (TMSi, Oldenzaal, Netherlands) and a TMS-compatible 64-electrode cap (ANT Neuro, Enschede, Netherlands). The EEG cap stayed in place during the whole day. Impedances were kept below $5 \mathrm{k} \Omega$. The ground electrode was placed between electrode positions $\mathrm{Fz}$ and Fpz. We used a common average reference for the recordings. A single TMS pulse produced a stimulation artifact of 1-2 millivolts, lasting approximately $3 \mathrm{~ms}$. To determine the hotspot and RMT, surface electrodes were placed in a belly-tendon montage over the right and left ADM muscle. The ground electrode was placed on the upper side of the wrist. We recorded the EMG using an additional amplifier (TMSi, Oldenzaal, Netherlands) connected to the EEG amplifier, ensuring synchronized measurements. The EEG and EMG signals were low-pass filtered with an anti-aliasing filter with a cut-off frequency of $550 \mathrm{~Hz}$ and sampled at $2048 \mathrm{~Hz}$. 


\section{Evoked potential analysis}

EMG and EEG analysis was performed using Matlab (The Mathworks, Natick, MA, USA). To analyze the MEP, peak-to-peak amplitudes were calculated and averaged per session. Trials containing muscle pre-activation, defined as EMG activity larger than $50 \mu \mathrm{V}$ in the $50 \mathrm{~ms}$ preceding a single pulse, were excluded.

TMS evoked potentials were analyzed using the common average reference. Trials were defined from $50 \mathrm{~ms}$ before to $300 \mathrm{~ms}$ after every TMS pulse, resulting in 75 trials for both targets and each session. We applied single-trial principal component analysis (PCA) to remove the first large TMS artifact, caused by the magnetic pulse, and the second TMS artifact, believed to be caused by muscle activation on the scalp. A detailed description of this PCA method can be found in a previous study (ter Braack et al. 2013). In short, we performed PCA using 40 calculated components on each individual trial, with the first component having the largest variance and the $40^{\text {th }}$ component having the lowest variance. We then removed the first four components, containing the large amplitude artifacts, from the trial to obtain a signal which is almost artifact-free. After PCA, the trials were filtered with a fourth order Butterworth bandpass filter between 1 and $45 \mathrm{~Hz}$ and averaged per session.

To investigate the variation of the P30 and N100 amplitude during the day, we determined the standard deviation at the latency of the maximum amplitude of both components at electrode $\mathrm{Cz}$. The standard deviation was determined for the response on group level for each session and then averaged over sessions, resulting in an average inter-individual variation of the P30 and N100 amplitude during the day. We also determined this standard deviation for each individual subject for the average response over all five sessions and then averaged over subjects, resulting in an average intra-individual variation of the P30 and N100 amplitude during the day.

\section{Statistical analysis}

For all statistical analyses a $p$-value below 0.05 was considered statistically significant, except when a correction for multiple comparisons was applied.

RMT and mean MEP amplitude between the five sessions were compared using oneway repeated measures ANOVA with Greenhouse-Geisser correction for both left and right motor cortex stimulation. Two-way repeated measures ANOVA was used to test for differences between both hemispheres. Since RMT was occasionally 
above the maximum output of the stimulator, and statistical analysis of bounded variables is challenging, those subjects were excluded for RMT statistics. A total of thirteen and fifteen subjects were included in the RMT analysis for the left and right hemispheres, respectively.

We compared the standard deviation of the P30 and N100 on group level (five sessions) with the standard deviation of the P30 and N100 on single subject level during the day (16 subjects) using an independent $t$-test.

All subjects were included in the TEP statistics. To compare the reproducibility of the total TEP waveform $(0-300 \mathrm{~ms})$ between the five sessions, we used multiple dependent $t$-tests at the electrode level. A cluster-based permutation analysis (Maris and Oostenveld 2007) was applied, as implemented in FieldTrip (http://fieldtrip.fcdonders.nl/). In short, a dependent $t$-test comparing the TEPs from the five sessions was performed for each time sample and each EEG electrode. Only $t$-values with a clustering $p$-value $<0.05$ were considered for clustering. Clustering of $t$-values was based on adjacent time bins and neighboring electrodes. Within each cluster, the $t$-values of the included electrodes were summed, and this sum was used for statistical comparison. A permutation test was performed, randomly assigning the TEPs from the 16 subjects to two different groups (for example session 1 and session 2 are now randomly shuffled) and repeating statistical testing for 1500 times. These permutation results are then combined to form a distribution of summed clusters $t$-values. Clusters in the original data set were considered to show a nonsignificant trend if less than $5 \%$ of the permutations in the distribution had a clusterlevel statistic larger than the statistic in the original data set, i.e. with an alpha $p$ value of $<0.05$. Only clusters with a $p$-value $<0.005$ were only considered statistically significant, as $p$-values were afterwards Bonferroni corrected for 10 comparisons (all sessions were compared to each other). The same procedure was repeated for the time-intervals $20-35 \mathrm{~ms}$ and $80-140 \mathrm{~ms}$ to evaluate the P30 and N100 components of the TEP in further detail.

\section{Results}

The mean RMT for the first session was 79\% for the left hemisphere and $78 \%$ for the right hemisphere (table 4.1). The RMT normalized with respect to the first session is presented in figure 4.1. The RMT showed no differences between the left and right hemisphere $(F(1.91,22.86)=0.09, p=0.90)$ and did not change significantly 
during the day (left hemisphere: $F(1.97,23.66)=0.41, p=0.67$; right hemisphere: $F(2.08,29.11)=1.99, p=0.15)$. The MEP amplitude showed no differences between the left and right hemisphere $(F(2.26,33.82)=0.16, p=0.88)$ and did not change significantly during the day (left hemisphere: $F(1.94,29.11)=1.50, p=0.24$; right hemisphere: $F(2.34,35.18)=1.94, p=0.15)$. In all subjects MEPs were evoked continuously, except in one where MEPs were absent (amplitude $<50 \mu \mathrm{V}$ ) for left hemisphere stimulation at 3:30PM and 6:00PM. The mean MEP amplitude normalized with respect to the first session is presented in figure 4.1.

Table 4.1. Resting motor thresholds and used TMS intensity (left / right) for all subjects.

\begin{tabular}{|c|c|c|c|c|c|c|}
\hline Subj. & Session 1 & Session 2 & Session 3 & Session 4 & Session 5 & $\begin{array}{c}\text { TMS } \\
\text { intensity }\end{array}$ \\
\hline 1 & $87 / 78$ & $89 / 77$ & $92 / 77$ & $95 / 76$ & $93 / 73$ & $96 / 86$ \\
\hline 2 & $80 / 65$ & $76 / 68$ & $86 / 67$ & $85 / 68$ & $84 / 68$ & $88 / 71$ \\
\hline 3 & $70 / 67$ & $72 / 67$ & $72 / 68$ & $71 / 72$ & $72 / 72$ & $77 / 74$ \\
\hline 4 & $70 / 82$ & $72 / 81$ & $72 / 80$ & $73 / 78$ & $73 / 83$ & $77 / 91$ \\
\hline 5 & $86 / 87$ & $79 / 88$ & $75 / 93$ & $75 / 93$ & $76 / 100$ & $95 / 96$ \\
\hline 6 & $65 / 61$ & $58 / 60$ & $62 / 61$ & $59 / 60$ & $58 / 57$ & $72 / 68$ \\
\hline $7 *$ & $97 * / 97 *$ & $95 / 99$ & $93 / 100$ & $95 / 99$ & $96 / 100$ & $100 / 100$ \\
\hline 8 & $80 / 84$ & $84 / 83$ & $80 / 81$ & $79 / 86$ & $78 / 86$ & $88 / 93$ \\
\hline $9 * \dagger$ & $94 * / 75$ & $100 / 75$ & $>100 / 85$ & $>100 / 82$ & $>100 / 90$ & $100 / 83$ \\
\hline 10 & $88 / 91$ & $89 / 85$ & $85 / 85$ & $87 / 82$ & $89 / 83$ & $97 / 100$ \\
\hline $11^{* \text { *新 }}$ & $\begin{array}{c}93 * / \\
>100 *\end{array}$ & $96 / 100$ & $\begin{array}{l}>100 / \\
>100\end{array}$ & $94 />100$ & $95 / 88$ & $100 / 100$ \\
\hline 12 & $74 / 70$ & $75 / 73$ & $77 / 71$ & $78 / 71$ & $83 / 70$ & $82 / 77$ \\
\hline 13 & $77 / 76$ & $64 / 65$ & $68 / 71$ & $66 / 76$ & $64 / 73$ & $85 / 85$ \\
\hline 14 & $84 / 73$ & $86 / 73$ & $88 / 74$ & $86 / 76$ & $92 / 74$ & $93 / 81$ \\
\hline 15 & $74 / 75$ & $78 / 74$ & $78 / 78$ & $80 / 78$ & $79 / 80$ & $82 / 83$ \\
\hline $16^{* \dagger}$ & $98 * / 93 *$ & $>100 / 93$ & 100 / 93 & 100 / 93 & $>100 / 94$ & $100 / 100$ \\
\hline
\end{tabular}

Resting motor threshold and applied TMS intensity during the protocol in \% of maximal stimulator output ( 1.5 tesla) for left / right hemisphere. * Protocol intensity of $110 \%$ RMT not possible, ${ }^{\dagger}$ subject not included in RMT analysis left hemisphere, ${ }^{\star}$ subject not included in RMT analysis right hemisphere. 

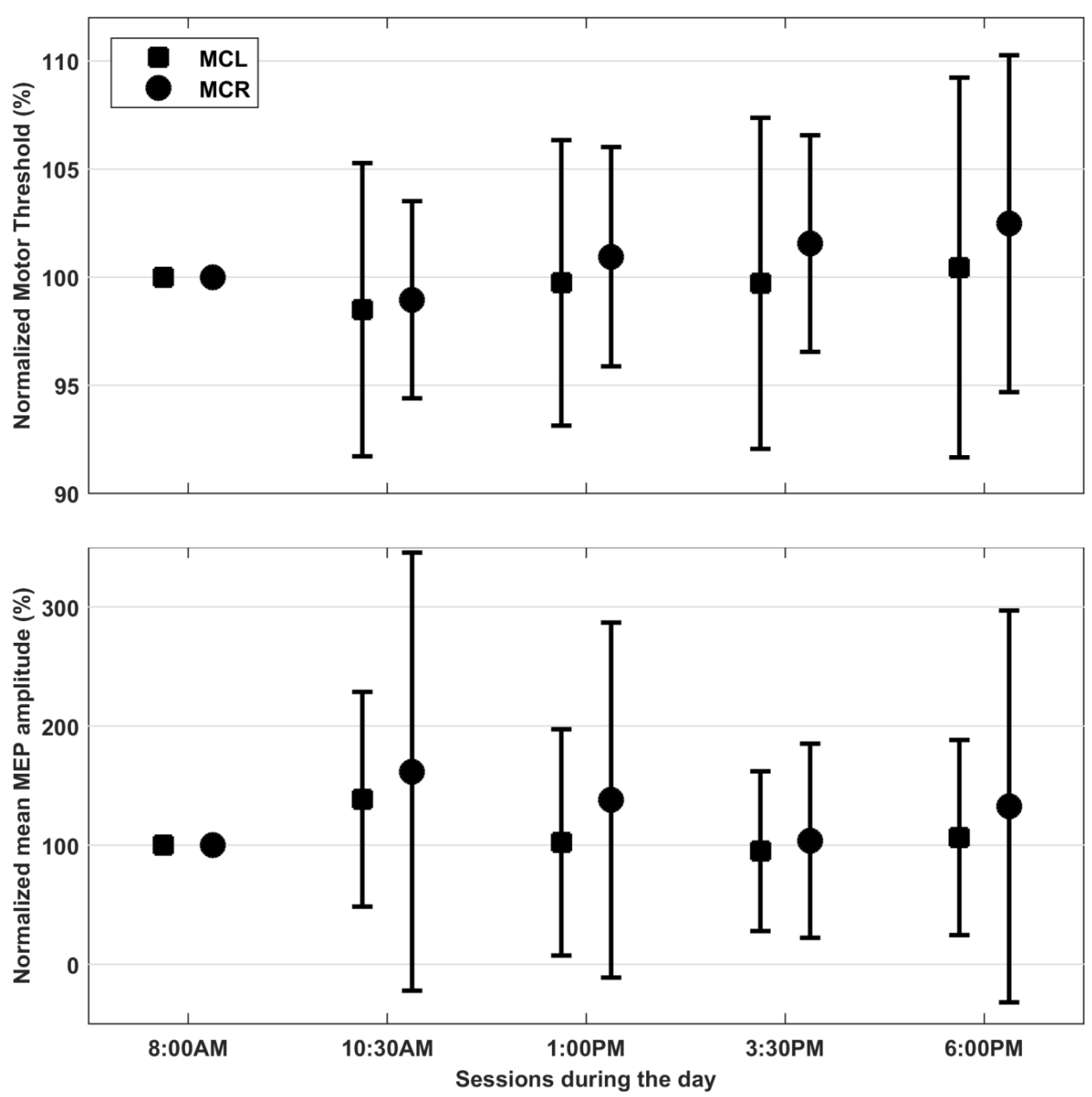

Figure 4.1. RMT and MEP variation during the day. The normalized RMT (top) and the normalized mean MEP amplitude (bottom) for the left (squares) and right (circles) hemisphere for all five sessions. The RMT and MEP amplitude were normalized with respect to the first session for every subject and then averaged over subjects. For RMT we excluded subjects with an RMT higher than $100 \%$ maximum stimulator output during one or more sessions, resulting in 13 subjects for the left hemisphere and 15 subjects for the right hemisphere. Errorbars indicate the standard deviation. 
Figure 4.2 shows the TEP for all five sessions at electrode $\mathrm{Cz}$ averaged over subjects after stimulating the left motor cortex. The response was very constant during the day, showing a similar waveform in all five sessions in each subject. We found no significant differences in the TEP between the five sessions for MCR stimulation on a group level. Also for MCL stimulation, the majority of sessions showed no significant differences, except for 10:30AM compared to 3:30PM ( $p=0.0046)$. One significant positive cluster was found left occipitally around $110-120 \mathrm{~ms}$, corresponding to a larger (more negative) amplitude of the N100 component at 3:30PM. When focusing on the N100, the same positive cluster was found to be significant (10:30AM compared to 3:30PM for MCL, $p=0.001$ ), located left occipitally.

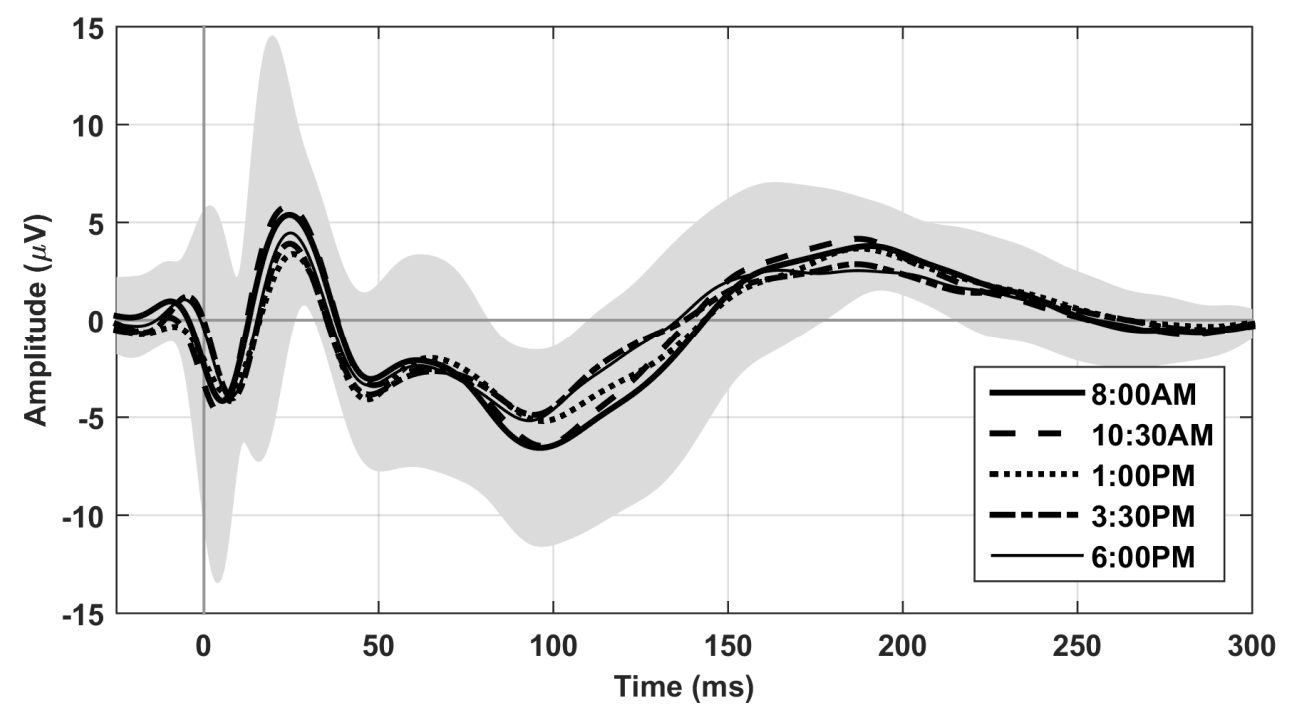

Figure 4.2. TEP on group level during the day. The TEP at electrode $\mathrm{Cz}$ on a group level during five sessions on one day after stimulating the left motor cortex. The TEP looks very similar during all five sessions, showing the typical components N15, P30, N45, P60, N100 and P180. The grey area represents the standard deviation on group level for the first session at 8:00AM. 
The electrode with the largest amplitude difference within the cluster (PO3) is shown in figure 4.3. When focusing on the P30, no additional significant clusters were found. In addition we found some non-significant trends for the N100 (8:00AM versus 3:30PM for MCL: positive cluster left occipitally (interval $0-300 \mathrm{~ms}(p=0.02)$; interval $80-140 \mathrm{~ms}(p=0.01))$, and 8:00AM versus 10:30AM for MCR: negative cluster left occipitally (interval $80-140 \mathrm{~ms}(p=0.02)$ ) and the P30 (10:30AM versus 1:00PM for MCL: negative cluster right frontally (interval $20-35 \mathrm{~ms}(p=0.02)$ ). The TEP after repeating the TMS protocol a week later also closely resembles the TEP from a week earlier (see figure 4.4).

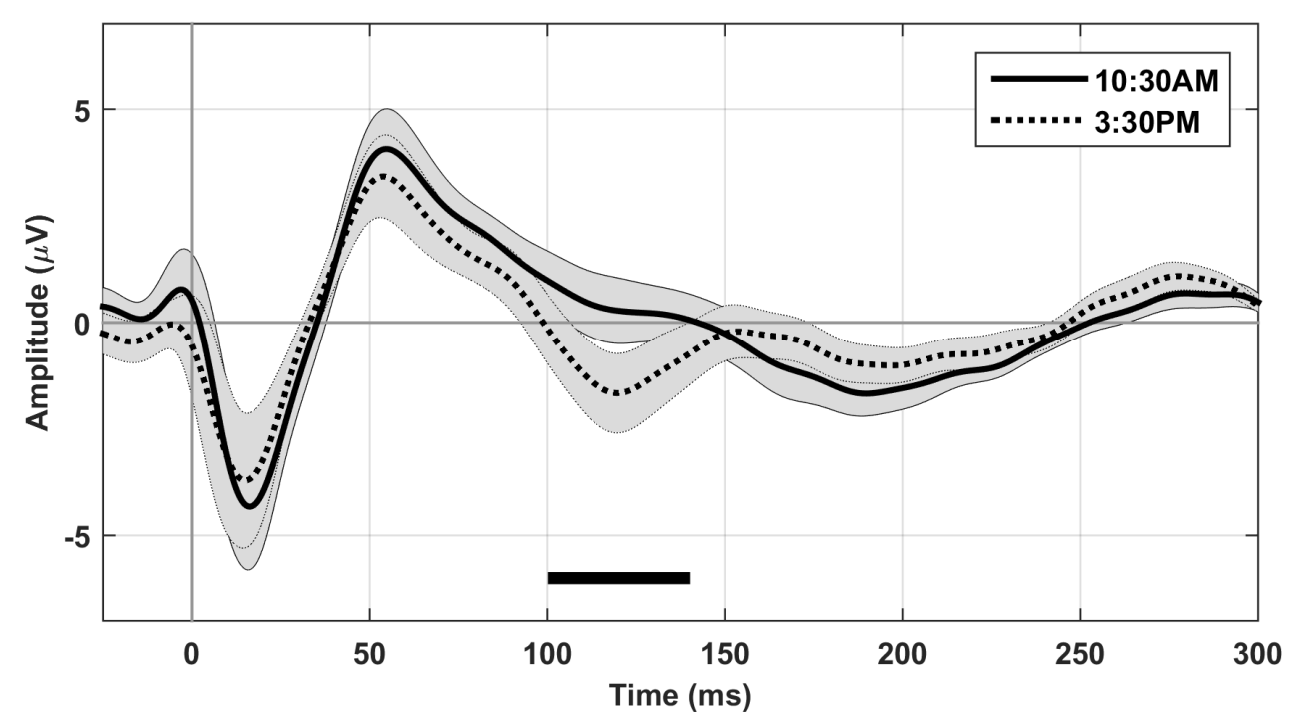

Figure 4.3. Difference in N100 between session 2 and 4. The TEP on group level in session 2 (10:30AM, solid line) and session 4 (3:30PM, dotted line) at electrode PO3 after stimulating the left motor cortex. The grey area represents the standard error. PO3 is the electrode with the largest difference in amplitude within the significant cluster, of which the duration is indicated by the black bar at the N100 component. 

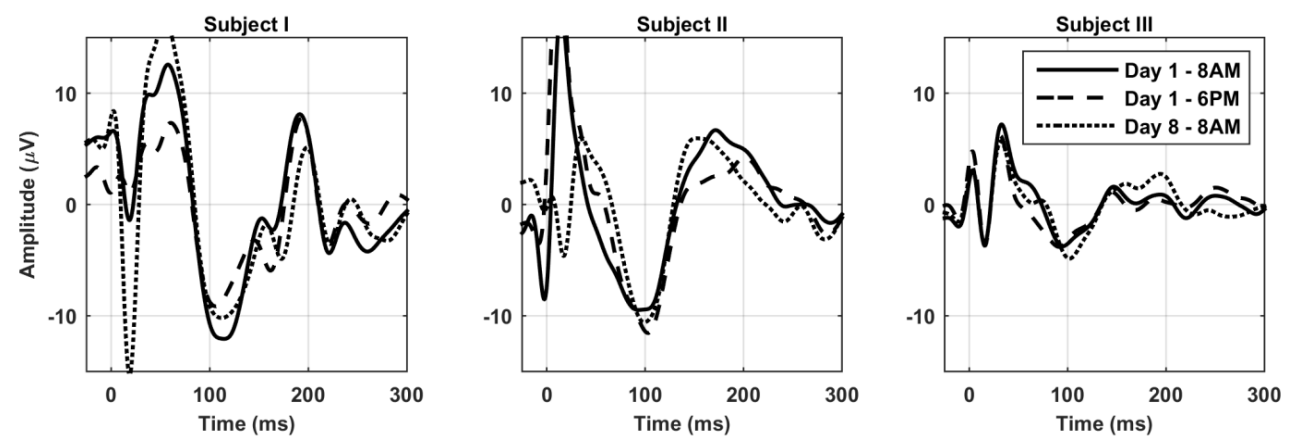

Figure 4.4. TEP after 1 week. The TEP at electrode $\mathrm{Cz}$ in 3 subjects after stimulating the right motor cortex at $8 \mathrm{AM}$ and $6 \mathrm{PM}$ on day 1 , and at $8 \mathrm{AM}$ after 1 week (day 8 ).

We calculated the mean TEP with standard deviation over all subjects in the five sessions. In figure 4.2 the mean TEP with $\mathrm{SD}$ at $\mathrm{Cz}$ is shown for left motor stimulation for session 1 . The average standard deviation of the N100 component at $\mathrm{Cz}$ on a group level during the day was $4.53 \mu \mathrm{V}$ (range 3.99-5.19 $\mu \mathrm{V}$ ) for MCL and $6.16 \mu \mathrm{V}$ (range 5.40-7.02 $\mu \mathrm{V}$ ) for MCR. The mean TEP with SD over five sessions for each single subject is shown in figure 4.5. The average standard deviation of the $\mathrm{N} 100$ component at $\mathrm{Cz}$ on a single subject level during the day was $2.01 \mu \mathrm{V}$ (range $0.40-4.27 \mu \mathrm{V}$ ) for MCL and 1.35 $\mu \mathrm{V}$ (range 0.27-3.11 $\mu \mathrm{V}$ ) for MCR. The average standard deviation of the $\mathrm{P} 30$ component at $\mathrm{Cz}$ on a group level during the day was 5.45 $\mu \mathrm{V}$ (range 4.36-6.98 $\mu \mathrm{V}$ ) for MCL and 4.42 $\mu \mathrm{V}$ (range 4.04-4.71 $\mu \mathrm{V}$ ) for MCR. The average standard deviation of the $\mathrm{P} 30$ component at $\mathrm{Cz}$ on a single subject level during the day was $2.98 \mu \mathrm{V}$ (range 0.58-8.48 $\mu \mathrm{V}$ ) for MCL and $1.14 \mu \mathrm{V}$ (range 0.36$2.73 \mu \mathrm{V}$ ) for MCR. Both for the N100 and the P30 the standard deviation on group level was significantly higher than the standard deviation on single subject level during the day (P30 MCL: $p=0.002$; P30 MCR, N100 MCL, N100 MCR: all $p<$ $0.001)$. 

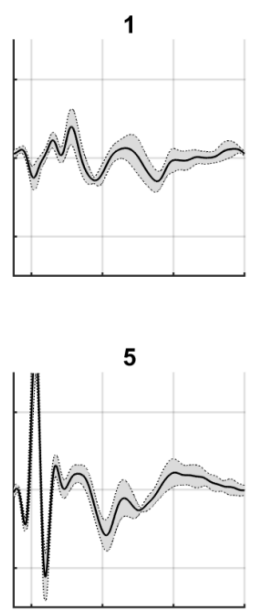

9

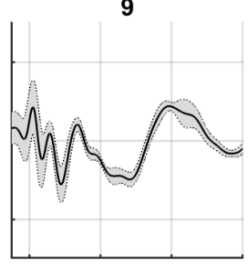

13

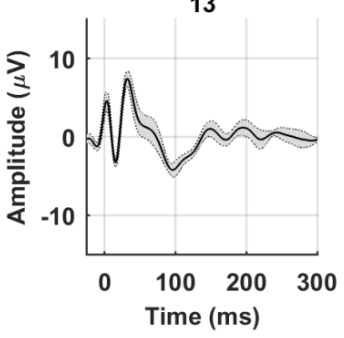

2

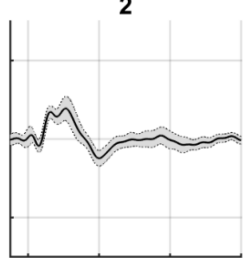

3
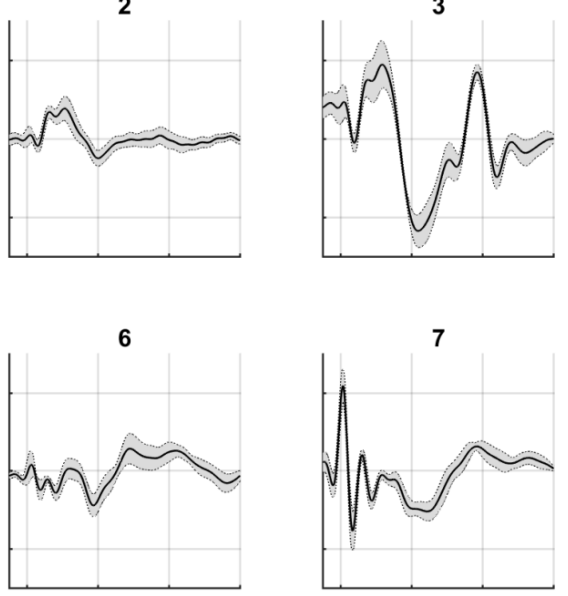

10

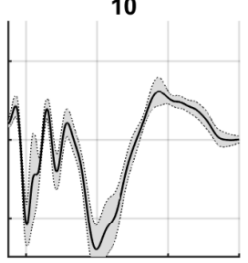

14

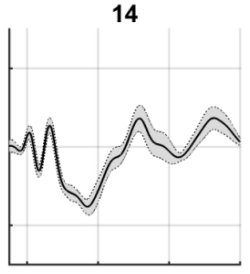

7

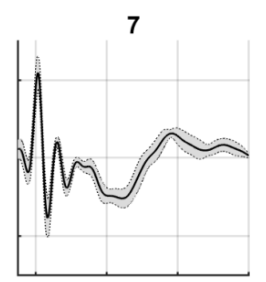

11

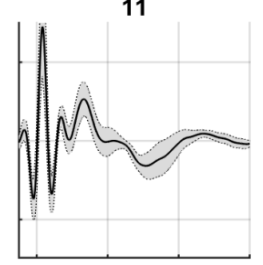

15

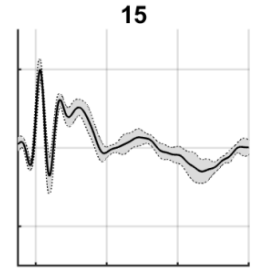

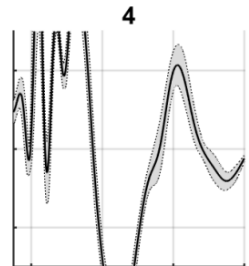

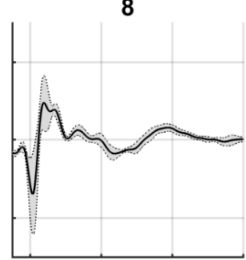

12

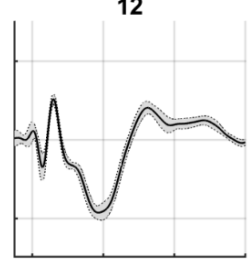

16

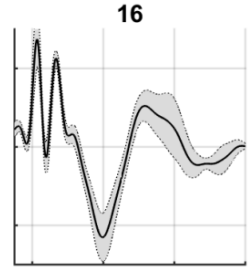

Figure 4.5. TEP on single subject level during the day. The mean TEP on electrode $\mathrm{Cz}$ during the day for all single subjects after stimulating the right motor cortex. The dotted lines represents the standard deviation. Numbers above the graphs indicate the subject number. 


\section{Discussion}

In this study we applied TMS-EEG five times during the day in healthy volunteers, and measured the RMT, MEP amplitude and the TEP. We found that both RMT and MEP amplitude do not change significantly during the day. The TEP remained largely constant, except for the N100 which was more pronounced at 3:30PM compared to 10:30AM. The inter-individual variation of the TEP within one session is significantly larger than the intra-individual variation during the day.

The absence of significant variation of the RMT between two sessions on one day has been reported before (Doeltgen and Ridding 2010; Lang et al. 2011; Tamm et al. 2009). Measurements at multiple time points in a $10 \mathrm{~h}$ period showed that the RMT stays constant (Koski et al. 2005), and this stability of the RMT during daytime is now confirmed by our results. Practically, this means that in healthy volunteers the RMT only has to be determined at the start of a TMS session, even when this TMS session takes very long or consists of multiple measurements within a day. In our data the MEP amplitude did not change over sessions, in line with a previous report measuring the MEP over a $24 \mathrm{~h}$ period (Strutton et al. 2003).

The TEP had a characteristic waveform in all subjects with recognizable components at 15-30-45-60-100-180 ms. This response was very constant during the day, with only the N100 after MCL stimulation showing a significant difference between the session at 10:30AM and 3:30PM on a group level. This cluster was located left occipitally, just as the two other N100 clusters that turned out to be non-significant after correction. Two earlier reports did not find a significant change of the first large component of the TEP (P30) during daytime (Huber et al. 2012; Ly et al. 2016). However, these authors did not analyze any other components. We evaluated the whole TEP waveform on all electrodes, and found a significant difference in a different component, the N100. All other components, including the P30, did not show significant differences. We only found a non-significant trend for the P30, with a larger amplitude at 1:00PM compared to 10:30AM, which is in line with previous findings of a non-significant increase of P30 amplitude during daytime (Huber et al. 2012; Ly et al. 2016). The TEP has shown to be reproducible after 1 week (Casarotto et al. 2010; Lioumis et al. 2009), similar to our observations in three subjects. 
Other TMS measures were also reported as being constant during the day, such as SICI (short interval cortical inhibition) and ICF (intra cortical facilitation) (Doeltgen and Ridding 2010; Lang et al. 2011; Pfutze et al. 2007). Both SICI and ICF are GABA-A mediated responses (Hanajima et al. 1998; Inghilleri et al. 1996; Kujirai et al. 1993). On the contrary, LICI (long interval cortical inhibition) as well as the CSP (cortical silent period) decreased in length during three sessions at 8AM, 2PM and 8PM (Lang et al. 2011), suggesting that the amount of inhibition decreases during the day. Earlier studies did not find a significant change in CSP during seven sessions between 8AM and 8PM (Koski et al. 2005) or between an evening and a morning session (Pfutze et al. 2007). LICI is a GABA-B mediated response (McDonnell et al. 2006; Pierantozzi et al. 2004; Werhahn et al. 1999), while CSP is thought to consist of a GABA-A mediated part and a GABA-B mediated part (Inghilleri et al. 1996; Kimiskidis et al. 2006; Siebner et al. 1998; Stetkarova and Kofler 2013). The N100 has also shown to be GABA-B mediated (Premoli et al. 2014), and in our results the N100 was larger in amplitude in the afternoon compared to the morning, suggesting an increase instead of decrease in inhibition. Taking these results together, it appears that GABA-A mediated TMS responses do not fluctuate during the day, while for the GABA-B mediated LICI and N100, and the partly GABA-B mediated CSP, inconsistent results are found.

Contradicting results are reported for the time-dependency of cortical excitability measured by LICI (long-interval cortical inhibition) in JME. Excitability was decreased in the afternoon compared to the morning in drug naive JME patients (Badawy et al. 2009), but no difference in excitability was reported in an earlier study (Pfutze et al. 2007). In addition, a difference in excitability between morning and afternoon could not be found in a group of focal epilepsy (mainly temporal lobe epilepsy) patients (Badawy et al. 2009). This inconsistency in TMS findings in healthy subjects and epilepsy patients may be explained by differences in methodology, for example the (lack of) control of different Zeitgebers or the heterogeneity of subjects with regard to morning type and evening type. True circadian or daytime variations may only be found using a constant routine methodology (Duffy and Dijk 2002). In any case, for a TMS readout to be of clinical use, the influence of normal, daily variations should be small compared to the influence of a disease or a medication. 
We further show that intra-individual variation during the day of the N100 and P30 amplitude was significantly smaller compared to the inter-individual variation within a session. This is in line with a previous report describing a large inter-individual variation in TMS measures (Koski et al. 2005). Large differences between subjects have also been described in recent publications evaluating the TEP before and after different (anti-epileptic) drugs (Premoli et al. 2017; Premoli et al. 2014).

Our study has a few limitations. Some subjects had a very high RMT at the start and therefore it was not possible to use a relative stimulation intensity of $110 \%$ during session 1. Because we compared the TEP between sessions, and not between subjects, and the TEP is also present at stimulation intensities below RMT (Komssi et al. 2004), this most likely had no effect on the results. We used the same stimulation intensity during all five sessions, even though there were small (nonsignificant) changes in RMT between sessions. MEP amplitude, directly dependent on RMT, has been related to the amplitude of the N15-P30 and N100 component (Mäki and Ilmoniemi 2010b; Paus et al. 2001) and to a late response around $300 \mathrm{~ms}$ (Fecchio et al. 2017). However, there were no significant differences in MEP amplitude between sessions. On the one hand the constant stimulation intensity may have influenced the TEP amplitude, since the relative stimulation intensity deviated from $110 \%$ in sessions 2-5. Still, this did not result in a difference in TEP waveform during the day, probably also because the relative stimulation intensity was sometimes above and sometimes below $110 \%$. On the other hand, keeping the stimulation intensity constant ensured that the contribution of auditory and somatosensory evoked potentials and muscle activation artifacts was similar for all sessions and did not influence our findings.

We used PCA to reduce the TMS pulse artifact and the muscle artifact from our data. At present, a large number of artifact rejection techniques have been used in TMSEEG. These consist of ICA (Hamidi et al. 2010; Iwahashi et al. 2008; Korhonen et al. 2011; Rogasch et al. 2014), PCA (Hernandez-Pavon et al. 2012; Mäki and Ilmoniemi 2010a; Rogasch et al. 2017) and other techniques (Casula et al. 2017; Litvak et al. 2007; Morbidi et al. 2007), all resulting in a reduction of TMS-related artifacts and thereby enabling TEP analysis. Although we have shown that with PCA the TMS pulse artifact and the muscle artifact can be reduced simultaneously (ter Braack et al. 2013), it may be worthwhile investigating whether the method can be further optimized. When increasingly more PCA components are removed, the TEP 
components are also decreased in amplitude (Mäki and Ilmoniemi 2010a; ter Braack et al. 2013). We now removed the first four components in all subjects, but it may be better to decide on the number of components to be removed on a single subject level, and possibly by also looking at the topographies of the components. The timelocked nature of both artifact and TEP poses a challenge when using ICA or PCA, and it would be interesting to compare these two techniques with other methods to decide on the best artifact rejection technique for TMS-EEG data.

In conclusion, we show that the RMT, MEP amplitude and TEP in healthy subjects are highly reproducible during daytime. No significant differences were found for both RMT and MEP amplitude, while only the N100 amplitude after left motor cortex stimulation was significantly larger at 3:30PM compared to 10:30AM. This implies that results from different sessions can be compared even though they are obtained at a different time of day. Large inter-individual differences still may cause difficulties in establishing normal ranges for TMS measures, which is important for developing clinical applications. 


\section{References}

Badawy RAB, Macdonell RAL, Jackson GD, Berkovic SF. "Why do seizures in generalized epilepsy often occur in the morning?". Neurology 2009; 73: 218-222

Barker AT, Jalinous R, Freeston IL. "Non-invasive magnetic stimulation of human motor cortex". Lancet 1985; 1: 1106-1107

Bauer PR, Kalitzin S, Zijlmans M, Sander JW, Visser GH. "Cortical excitability as a potential clinical marker of epilepsy: a review of the clinical application of transcranial magnetic stimulation". Int J Neural Syst 2014; 24: e1430001

Bonato C, Miniussi C, Rossini PM. "Transcranial magnetic stimulation and cortical evoked potentials: a TMS/EEG co-registration study". Clin Neurophysiol 2006; 117: 16991707

Casarotto S, Lauro LJR, Bellina V, Casali AG, Rosanova M, Pigorini A et al. "EEG responses to TMS are sensitive to changes in the perturbation parameters and repeatable over time". PLoS One 2010; 5: e10281

Casula EP, Bertoldo A, Tarantino V, Maiella M, Koch G, Rothwell JC et al. "TMS-evoked long-lasting artefacts: A new adaptive algorithm for EEG signal correction". Clin Neurophysiol 2017; 128: 1563-1574

Chen R, Cros D, Curra A, Lazzaro VD, Lefaucheur J-P, Magistris MR et al. "The clinical diagnostic utility of transcranial magnetic stimulation: report of an IFCN committee". Clin Neurophysiol 2008; 119: 504-532

Doeltgen SH, Ridding MC. "Behavioural exposure and sleep do not modify corticospinal and intracortical excitability in the human motor system". Clin Neurophysiol 2010; 121 : 448-452

Duffy JF, Dijk DJ. "Getting through to circadian oscillators: Why use constant routines?". J Biol Rhythm 2002; 17: 4-13

Durazzo TS, Spencer SS, Duckrow RB, Novotny EJ, Spencer DD, Zaveri HP. "Temporal distributions of seizure occurrence from various epileptogenic regions". Neurology 2008; 70: 1265-1271

Engel J, Jr. "Progress in epilepsy Reducing the treatment gap and the promise of biomarkers". Curr Opin Neurol 2008; 21: 50-54

Fecchio M, Pigorini A, Comanducci A, Sarasso S, Casarotto S, Premoli I et al. "The spectral features of EEG responses to transcranial magnetic stimulation of the primary motor cortex depend on the amplitude of the motor evoked potentials". PLoS One 2017; 12: e0184910

Ferreri F, Pasqualetti P, Määttä S, Ponzo D, Ferrarelli F, Tononi G et al. "Human brain connectivity during single and paired pulse transcranial magnetic stimulation". Neuroimage 2011; 54: 90-102 
Hamidi M, Slagter HA, Tononi G, Postle BR. "Brain responses evoked by high-frequency repetitive transcranial magnetic stimulation: an event-related potential study". Brain Stimul 2010; 3: 2-14

Hanajima R, Ugawa Y, Terao Y, Sakai K, Furubayashi T, Machii K, Kanazawa I. "Pairedpulse magnetic stimulation of the human motor cortex - differences among Iwaves". Journal of Physiology 1998; 509: 607-618

Hernandez-Pavon JC, Metsomaa J, Mutanen T, Stenroos M, Mäki H, Ilmoniemi RJ, Sarvas J. "Uncovering neural independent components from highly artifactual TMSevoked EEG data". Journal of Neuroscience Methods 2012; 209: 144-157

Hofstra WA, de Weerd AW. "The circadian rhythm and its interaction with human epilepsy: a review of literature". Sleep Med Rev 2009; 13: 413-420

Hofstra WA, Spetgens WP, Leijten FS, van Rijen PC, Gosselaar P, van der Palen J, de Weerd AW. "Diurnal rhythms in seizures detected by intracranial electrocorticographic monitoring: an observational study". Epilepsy Behav 2009; 14: 617-621

Huber R, Maki H, Rosanova M, Casarotto S, Canali P, Casali AG et al. "Human Cortical Excitability Increases with Time Awake". Cerebral Cortex 2012; 23: 1-7

Ilmoniemi RJ, Kičić D. "Methodology for combined TMS and EEG". Brain Topogr 2010; 22: $233-248$

Inghilleri M, Berardelli A, Marchetti P, Manfredi M. "Effects of diazepam, baclofen and thiopental on the silent period evoked by transcranial magnetic stimulation in humans". Exp Brain Res 1996; 109: 467-472

Iwahashi M, Arimatsu T, Ueno S, Iramina K. "Differences in evoked EEG by transcranial magnetic stimulation at various stimulus points on the head". Conf Proc IEEE Eng Med Biol Soc 2008; 2008: 2570-2573

Kerwin LJ, Keller CJ, Wu W, Narayan M, Etkin A. "Test-retest reliability of transcranial magnetic stimulation EEG evoked potentials". Brain Stimul 2018; 11: 536-544

Kimiskidis VK. "TMS coupled with EEG Biomarker of the future". Revue Neurologique 2016; 172: 123-126

Kimiskidis VK, Papagiannopoulos S, Kazis DA, Sotirakoglou K, Vasiliadis G, Zara F et al. "Lorazepam-induced effects on silent period and corticomotor excitability". Exp Brain Res 2006; 173: 603-611

Komssi S, Kähkönen S, Ilmoniemi RJ. "The effect of stimulus intensity on brain responses evoked by transcranial magnetic stimulation". Hum Brain Mapp 2004; 21: 154-164

Korhonen RJ, Hernandez-Pavon JC, Metsomaa J, Mäki H, Ilmoniemi RJ, Sarvas J. "Removal of large muscle artifacts from transcranial magnetic stimulation-evoked EEG by independent component analysis". Med Biol Eng Comput 2011; 49: 397-407

Koski L, Schrader LM, Wu AD, Stern JM. "Normative data on changes in transcranial magnetic stimulation measures over a ten hour period". Clin Neurophysiol 2005; 116: 2099-2109 
Kujirai T, Caramia MD, Rothwell JC, Day BL, Thompson PD, Ferbert A et al. "Corticocortical inhibition in human motor cortex". Journal of Physiology 1993; 471: 501-519

Lang N, Rothkegel H, Reiber H, Hasan A, Sueske E, Tergau F et al. "Circadian Modulation of GABA-Mediated Cortical Inhibition". Cereb Cortex 2011; 21: 2299-2306

Lioumis P, Kičić D, Savolainen P, Mäkelä JP, Kähkönen S. "Reproducibility of TMS-Evoked EEG responses". Hum Brain Mapp 2009; 30: 1387-1396

Litvak V, Komssi S, Scherg M, Hoechstetter K, Classen J, Zaaroor M et al. "Artifact correction and source analysis of early electroencephalographic responses evoked by transcranial magnetic stimulation over primary motor cortex". Neuroimage 2007; 37: $56-70$

Ly JQ, Gaggioni G, Chellappa SL, Papachilleos S, Brzozowski A, Borsu C et al. "Circadian regulation of human cortical excitability". Nat Commun 2016; 7: e11828

Mäki H, Ilmoniemi RJ. "Projecting out muscle artifacts from TMS-evoked EEG". Neuroimage 2010a; 54: 2706-2710

Mäki H, Ilmoniemi RJ. "The relationship between peripheral and early cortical activation induced by transcranial magnetic stimulation". Neurosci Lett 2010b; 478: 24-28

Manganotti P, del Felice A. "New perspectives in transcranial magnetic stimulation: Epilepsy, consciousness and the perturbational approach". Behav Neurol 2013; 27: $155-167$

Maris E, Oostenveld R. "Nonparametric statistical testing of EEG- and MEG-data". J Neurosci Methods 2007; 164: 177-190

McDonnell MN, Orekhov Y, Ziemann U. "The role of GABA(B) receptors in intracortical inhibition in the human motor cortex". Exp Brain Res 2006; 173: 86-93

Miniussi C, Thut G. "Combining TMS and EEG Offers New Prospects in Cognitive Neuroscience". Brain Topogr 2010; 22: 249-256

Mirzoev A, Bercovici E, Stewart LS, Cortez MA, Snead OC, 3rd, Desrocher M. "Circadian profiles of focal epileptic seizures: a need for reappraisal". Seizure 2012; 21: 412416

Morbidi F, Garulli A, Prattichizzo D, Rizzo C, Manganotti P, Rossi S. "Off-line removal of TMS-induced artifacts on human electroencephalography by Kalman filter". J Neurosci Methods 2007; 162: 293-302

Nobelprize.org The 2017 Nobel Prize in Physiology or Medicine - Press Release. Nobel Media AB 2014. http://www.nobelprize.org/nobel_prizes/medicine/laureates/2017/press.html Accessed Web. 5 Nov 2017.

Paus T, Sipila PK, Strafella AP. "Synchronization of neuronal activity in the human primary motor cortex by transcranial magnetic stimulation: an EEG study". J Neurophysiol 2001; 86: 1983-1990 
Pavlova MK, Shea SA, Bromfield EB. "Day/night patterns of focal seizures". Epilepsy \& Behavior 2004; 5: 44-49

Pfutze M, Reis J, Haag A, John D, Hattemer K, Oertel WH et al. "Lack of differences of motorcortical excitability in the morning as compared to the evening in juvenile myoclonic epilepsy - a study using transcranial magnetic stimulation". Epilepsy Res 2007; 74: 239-242

Pierantozzi M, Marciani MG, Palmieri MG, Brusa L, Galati S, Caramia MD et al. "Effect of Vigabatrin on motor responses to transcranial magnetic stimulation: an effective tool to investigate in vivo GABAergic cortical inhibition in humans". Brain Res 2004; 1028: 1-8

Premoli I, Biondi A, Carlesso S, Rivolta D, Richardson MP. "Lamotrigine and levetiracetam exert a similar modulation of TMS-evoked EEG potentials". Epilepsia 2017; 58: 4250

Premoli I, Castellanos N, Rivolta D, Belardinelli P, Bajo R, Zipser C et al. "TMS-EEG signatures of GABAergic neurotransmission in the human cortex". J Neurosci 2014; 34: 5603-5612

Rogasch NC, Sullivan C, Thomson RH, Rose NS, Bailey NW, Fitzgerald PB et al. "Analysing concurrent transcranial magnetic stimulation and electroencephalographic data: A review and introduction to the open-source TESA software". Neuroimage 2017; 147: 934-951

Rogasch NC, Thomson RH, Farzan F, Fitzgibbon BM, Bailey NW, Hernandez-Pavon JC et al. "Removing artefacts from TMS-EEG recordings using independent component analysis: importance for assessing prefrontal and motor cortex network properties". Neuroimage 2014; 101: 425-439

Rossi S, Hallett M, Rossini PM, Pascual-Leone A, Safety of TMSCG. "Safety, ethical considerations, and application guidelines for the use of transcranial magnetic stimulation in clinical practice and research". Clin Neurophysiol 2009; 120: 20082039

Rossini PM, Barker AT, Berardelli A, Caramia MD, Caruso G, Cracco RQ et al. "Noninvasive electrical and magnetic stimulation of the brain, spinal cord and roots: basic principles and procedures for routine clinical application. Report of an IFCN committee". Electroencephalogr Clin Neurophysiol 1994; 91: 79-92

Siebner HR, Dressnandt J, Auer C, Conrad B. "Continuous intrathecal baclofen infusion indced a marked increase of the transcranially evoked silent period". Muscle \& Nerve 1998; 21: 1209-1212

Stetkarova I, Kofler M. "Differential effect of baclofen on cortical and spinal inhibitory circuits". Clin Neurophysiol 2013; 124: 339-345

Strutton P, Catley M, Davey N. "Stability of corticospinal excitability and grip force in intrinsic hand muscles in man over a 24-h period". Physiology \& Behavior 2003; 79: 679-682 
Tamm AS, Lagerquist O, Ley AL, Collins DF. "Chronotype influences diurnal variations in the excitability of the human motor cortex and the ability to generate torque during a maximum voluntary contraction". J Biol Rhythms 2009; 24: 211-224

ter Braack EM, de Jonge B, van Putten MJ. "Reduction of TMS induced artifacts in EEG using principal component analysis". IEEE Trans Neural Syst Rehabil Eng 2013; 21: 376-382

ter Braack EM, de Vos CC, van Putten MJ. "Masking the Auditory Evoked Potential in TMSEEG: A Comparison of Various Methods". Brain Topography 2015; 28: 520-528

ter Braack EM, Koopman AW, van Putten MJ. "Early TMS evoked potentials in epilepsy: A pilot study". Clin Neurophysiol 2016; 127: 3025-3032

van Campen JS, Valentijn FA, Jansen FE, Joels M, Braun KP. "Seizure occurrence and the circadian rhythm of cortisol: a systematic review". Epilepsy Behav 2015; 47: 132137

Werhahn KJ, Kunesch E, Noachtar S, Benecke R, Classen J. "Differential effects on motorcortical inhibition induced by blockade of GABA uptake in humans". Journal of Physiology 1999; 517: 591-597 


\section{Chapter 5 \\ Early TMS evoked potentials in epilepsy: a pilot study}

Esther M. ter Braack, Anne-Wil E. Koopman, Michel J.A.M. van Putten Clinical Neurophysiology 2016;127:3025-3032 


\section{Abstract}

Objective: To explore if the TMS evoked potential is different in patients with epilepsy compared to healthy subjects.

Methods: Eighteen healthy subjects and thirteen epilepsy patients participated in this study. Single TMS pulses were applied to the left and right motor cortex. For each target we applied 75 pulses at $110 \%$ of the resting motor threshold (RMT), and continuously measured the EEG. Resting motor threshold and the TMS evoked potential (TEP) were compared between patients and healthy subjects.

Results: Epilepsy patients had a higher left RMT than healthy subjects (88.5\% vs. $81.8 \%, p=0.048)$. For left motor cortex stimulation, the N100 was larger in amplitude in epilepsy patients than in healthy subjects $(p=0.0073)$. For right motor cortex stimulation, the P180 was larger in amplitude in epilepsy patients than in healthy subjects $(p=0.006)$. The differences in these late TEP components were localized in the centro-parietal areas. No significant differences were found for other TEP components.

Conclusions: In this pilot study, we found a significant higher MT and higher TEP amplitudes in epilepsy patients compared to healthy subjects.

Significance: Changes in cortical excitability may assist in epilepsy diagnostics or evaluation of the efficacy of anti-epileptic drugs. 


\section{Introduction}

Epilepsy is characterized by an enduring predisposition to generate epileptic seizures (Fisher et al. 2005), resulting from an imbalance between excitatory and inhibitory activity (McCormick and Contreras 2001). This imbalance is also reflected by the presence of interictal discharges. Such abnormal synchrony may occur in response to external stimuli, for instance in patients with photosensitive epilepsy, showing epileptiform discharges during photic stimulation (Verrotti et al. 2012). In this context, one may define cortical excitability as the responsiveness of a network of cortical neurons to a certain input stimulus, e.g. light, sound or an electrical current.

With transcranial magnetic stimulation (TMS), the excitability of the cortex can be measured using a short magnetic pulse as the input stimulus. Traditionally, the motor cortex is stimulated using single pulses, and the motor threshold (MT) and cortical silent period (CSP) are used as measures of excitability (Badawy et al. 2012; Tassinari et al. 2003). A recent overview showed that different drugs used in epilepsy management result in differences in MT, where excitability was mostly reduced after taking AEDs (Ziemann et al. 2015). However, despite a large number of TMS studies, no consistent differences in MT or CSP between epilepsy patients and healthy subjects were found (Bauer et al. 2014). Importantly, MT and CSP not only reflect cortical excitability, but also the excitability of the spinal cord, peripheral nerves and muscles; where it is not straightforward to define the contribution of the various involved systems.

Combining TMS with EEG (TMS-EEG) has only recently become available. This technique offers a more direct measurement of the brain's response to a TMS pulse and gives the opportunity to record responses after stimulating other areas than the motor cortex (Ilmoniemi and Kičić 2010). The TMS evoked potential (TEP) is obtained by averaging the EEG over a large number of single TMS pulses. When stimulating the (left or right) motor cortex, the TEP shows characteristic components at 15, 30, 45, 60, 100 and $180 \mathrm{~ms}$ after the stimulus (Ilmoniemi and Kičić 2010; ter Braack et al. 2013), although earlier components at 7-10 and 13-14 ms have been reported as well (Bonato et al. 2006; Ferreri et al. 2011).

The origin of the different TEP components remains largely unclear, although there have been attempts to elucidate the different underlying mechanisms. The N15-P30 complex shows a correlation with the amplitude of the motor evoked potential (Mäki 
and Ilmoniemi 2010) and probably reflects excitation (Esser et al. 2006). On the other hand, the N100 is strongly related to inhibitory processes, with a reduction in amplitude when a subject is preparing for a movement (Kičić et al. 2008; Nikulin et al. 2003). The N100 correlates with the duration of the CSP (Farzan et al. 2013). The P60 and N100 both increased after a repetitive TMS paradigm which was aimed at increasing inhibition (Casula et al. 2014). Recently it has been shown that GABAergic drugs that enhance inhibition result in changes in the N45 and N100 (Premoli et al. 2014a).

TMS-EEG seems to be a promising technique to explore in epilepsy patients, where the various components of the TEP may serve as a new biomarker for cortical excitability (Kimiskidis et al. 2014). To our knowledge, only two studies have been published which compare the TEP after motor cortex stimulation in epilepsy patients and healthy controls (Del Felice et al. 2011; Julkunen et al. 2013). The P30 was higher in amplitude and the N100 and P180 were lower in amplitude in patients with Unverricht-Lundborg type progressive myoclonus epilepsy (EPM1) compared to healthy controls (Julkunen et al. 2013). Patients with juvenile myoclonic epilepsy (JME) as well as healthy subjects showed an increase in amplitude of the TEP components around 100 and $180 \mathrm{~ms}$ after sleep deprivation, but this increase was significantly larger in the JME patients (Del Felice et al. 2011). Although both studies show differences in the N100 component between patients and controls, the N100 was decreased in EPM1 patients and increased in JME patients.

To explore TMS-EEG as a tool for epilepsy research, more basic patient studies are needed to explore the specific characteristics of the TEP in epilepsy patients and healthy subjects. In this pilot study, we measured the resting motor threshold (RMT) and evaluated the TEP after motor cortex stimulation and compared these measures between a heterogeneous group of epilepsy patients and healthy subjects.

\section{Methods}

The experimental protocol was approved by the local ethics committee (Medisch Spectrum Twente, Enschede, the Netherlands) and was in accordance with the declaration of Helsinki. Written informed consent was obtained from all included subjects. We followed the guidelines for the use of TMS in clinical practice and research (Rossi et al. 2009). 


\section{Subjects}

Eighteen healthy subjects ( 11 males, mean age 28 years, range $20-54$ years, all righthanded) and thirteen epilepsy patients (4 males, mean age 26 years, range 19-42 years, all right-handed) participated in this study. Patients with clinically confirmed epilepsy were randomly selected from the database of the department of Clinical Neurophysiology at Medisch Spectrum Twente. The diagnosis of epilepsy was made by an experienced neurologist based on the patient history and routine EEG recordings. We excluded patients who experienced seizures in the month before the TMS measurement. Subjects with implanted devices or metal objects in their body were excluded. In addition, we excluded healthy subjects with a history of epilepsy or epileptic events, as well as healthy subjects with family members having epilepsy. Patient characteristics are shown in table 5.1.

\section{Experimental conditions}

Subjects were seated in a chair, with their hands in a relaxed position. They kept their eyes open and focused on a marked point on the wall. During TMS-EEG, all subjects wore protective earplugs, and noise created from the coil click was played through headphones at $95 \mathrm{~dB}$ to mask the sound of the TMS pulses (ter Braack et al. 2015). In addition, a thin layer of foam was placed between the coil and head of the subject to minimize bone conduction.

\section{TMS targeting}

Single biphasic TMS pulses, with pulse duration of $400 \mu$ s and inter-pulse interval of approximately 4 seconds, were delivered manually. We used a $70 \mathrm{~mm}$ figure-ofeight air film coil and a Rapid ${ }^{2}$ stimulator (The Magstim Company Ltd, Whitland, United Kingdom) with a maximum output of 1.5 tesla. The coil was placed tangentially over the hot-spot of the abductor digiti minimi (ADM) muscle in the left and right hemisphere, with the handle pointing backwards and laterally at an angle $45^{\circ}$ away from the midline. We applied 75 TMS pulses at both targets with a stimulation intensity of $110 \%$ RMT. Positioning of the coil was achieved using a robot-navigated system (Smartmove, ANT Neuro, Enschede, Netherlands), as described in our previous study (ter Braack et al. 2013). In all healthy subjects, a 1.5 tesla MRI scan of the head was available to create a subject-specific head model, making the set-up procedure more rapid. In the patients, the available MRI scans 
could not be used for TMS targeting due to technical problems, and a standard head model was used for navigating the TMS coil. This had no consequences for determining the hot spot, as this was done manually, or automatic placement of the coil.

\begin{tabular}{|c|c|c|c|c|c|c|c|c|}
\hline$\#$ & Sex & Age & $\begin{array}{l}\text { Type of } \\
\text { epilepsy }\end{array}$ & $\begin{array}{l}\text { Duration } \\
\text { (years) }\end{array}$ & $\begin{array}{l}\text { Epileptiform } \\
\text { abnormalities } \\
\text { (scalp EEG) }\end{array}$ & MRI & Medication & SF \\
\hline 1 & $\mathrm{M}$ & 40 & Generalized & 5 & Normal EEG & $\begin{array}{l}\text { Lesions } \\
\text { L: } \mathrm{P}+\mathrm{T}\end{array}$ & Valproic acid & $\mathrm{Y}$ \\
\hline 2 & $\mathrm{~F}$ & 20 & Generalized & 9 & $\begin{array}{l}\mathrm{G} \\
\mathrm{L}: \mathrm{T}\end{array}$ & $\begin{array}{l}\text { Glioma } \\
\text { L: T }\end{array}$ & Lacosamide & $\mathrm{N}$ \\
\hline 3 & $\mathrm{~F}$ & 20 & Generalized & 3 & Normal EEG & Normal & Lamotrigine & $\mathrm{N}$ \\
\hline 4 & $\mathrm{~F}$ & 26 & $\begin{array}{l}\text { Generalized } \\
\text { (JME) }\end{array}$ & $>10$ & $\mathrm{G}$ & $\begin{array}{l}\text { No } \\
\text { MRI }\end{array}$ & Lamotrigine & $\mathrm{Y}$ \\
\hline 5 & $\mathrm{M}$ & 19 & Focal & 3 & $\mathrm{R}: \mathrm{T}$ & Normal & Valproic acid & $\mathrm{Y}$ \\
\hline 6 & $\mathrm{~F}$ & 19 & Generalized & 8 & L: FT & Normal & Lamotrigine & $\mathrm{Y}$ \\
\hline 7 & $\mathrm{~F}$ & 42 & $\begin{array}{l}\text { Generalized } \\
\text { (possible } \\
\text { JME) }\end{array}$ & 4 & Normal EEG & Normal & $\begin{array}{l}\text { Lamotrigine } \\
\text { Lacosamide }\end{array}$ & $\mathrm{N}$ \\
\hline 8 & $\mathrm{~F}$ & 20 & Generalized & 7 & $\mathrm{~L}+\mathrm{R}: \mathrm{T}$ & Normal & Lamotrigine & $\mathrm{Y}$ \\
\hline 9 & $\mathrm{~F}$ & 19 & $\begin{array}{l}\text { Generalized } \\
\text { (JME) }\end{array}$ & 5 & $\mathrm{G}$ & Normal & Lamotrigine & $\mathrm{Y}$ \\
\hline 10 & $\mathrm{~F}$ & 31 & Generalized & 21 & $\mathrm{G}$ & Normal & $\begin{array}{l}\text { Ethosuximide } \\
\text { Levetiracetam }\end{array}$ & $\mathrm{Y}$ \\
\hline 11 & $\mathrm{~F}$ & 31 & Focal & 1 & L: FT & Normal & Valproic acid & $\mathrm{Y}$ \\
\hline 12 & $\mathrm{M}$ & 31 & Generalized & 22 & L+R: CT & Normal & Oxcarbazepine & $\mathrm{N}$ \\
\hline 13 & $\mathrm{M}$ & 25 & Generalized & 11 & Normal EEG & Normal & Valproic acid & $\mathrm{N}$ \\
\hline \multicolumn{9}{|c|}{$\begin{array}{l}\text { Duration = duration of epilepsy; JME }=\text { Juvenile myoclonic epilepsy; } \mathrm{SF}=\text { seizure free. } \mathrm{G}= \\
\text { generalized; } \mathrm{L}=\text { left } \mathrm{R}=\text { right } \mathrm{P}=\text { parietal; } \mathrm{T}=\text { temporal; } \mathrm{FT}=\text { fronto-temporal; } \mathrm{CT}=\text { centro- } \\
\text { temporal; } \mathrm{M}=\text { male } ; \mathrm{F}=\text { female } ; \mathrm{Y}=\text { yes } ; \mathrm{N}=\text { no. }\end{array}$} \\
\hline
\end{tabular}

\section{EEG and EMG recordings}

A 64-channel EEG was recorded continuously during TMS using a TMS compatible EEG cap (ANT Neuro, Enschede, the Netherlands) and full-band EEG amplifier (TMSi, Oldenzaal, the Netherlands). Electrode impedances were kept below 5 $\mathrm{kOhm}$. For the recordings, we used a sample frequency of $2048 \mathrm{~Hz}$ and the common average reference. The ground electrode was placed between Fz and FPz. A single TMS pulse produced a stimulation artifact of $1-2 \mathrm{mV}$, lasting for approximately 3 
ms. In the epilepsy patients, a 10-minute resting EEG was recorded before and after TMS. To determine the hot spot and RMT, surface electrodes were placed in a bellytendon montage over the left and right ADM muscle. The ground electrode was placed on the dorsal side of the wrist. The EMG was sampled at $2048 \mathrm{~Hz}$ and recorded using an additional amplifier (TMSi, Oldenzaal, Netherlands). RMT was defined as the lowest stimulus intensity that produced at least five MEPs of at least $50 \mu \mathrm{V}$ out of 10 consecutive stimuli (Rossini et al. 1994).

\section{Resting EEG analysis}

The resting EEGs in the epilepsy patients, and the EEGs during TMS in both epilepsy patients and healthy controls, were reviewed by an experienced EEG technician and a clinical neurophysiologist (MvP). They scored the EEGs for epileptiform abnormalities and had to reach consensus. Both reviewers were blinded to the subject category (patient or healthy subject).

\section{Evoked potential analysis}

EEG analysis was performed using Matlab (The Mathworks, Natick, MA, USA). The common average reference was used for analyzing the TMS evoked potentials. Trials were defined from 2 seconds before to 2 seconds after every TMS pulse, resulting in 75 trials of 4 seconds for both targets. Trials with eye-blinks were automatically rejected using a fixed threshold of $150 \mu \mathrm{V}$ for electrode channel $\mathrm{Cz}$, which resulted in at least 70 accepted trials for all targets.

We applied single-trial principal component analysis (PCA) to remove the first large TMS artifact, caused by the magnetic pulse, and the second TMS artifact, believed to be caused by muscle activation on the scalp (Mutanen et al. 2013). A detailed description of this PCA method can be found in a previous study (ter Braack et al. 2013). In short, we performed PCA using 40 calculated components on each individual trial. Based on the results from our previous study, we then removed the first four components to obtain an artifact-free signal. Figures S1-S4 showing the signal before and after PCA can be found in the supplemental material.

After PCA, the trials were averaged and filtered with a fourth order Butterworth bandpass filter between 1 and $80 \mathrm{~Hz}$ with an additional $50 \mathrm{~Hz}$ bandstop filter. To evaluate a possible relationship between stimulation intensity and amplitude of TEP components, the response measured at electrode $\mathrm{Cz}$ was selected for further analysis. 
The amplitudes of the TEP components P30, N100 and P180 were determined by manually selecting a latency window for each peak, based on visual inspection of the TEP, and then automatically detecting the largest absolute amplitude within that range. In figure S5-S6 in the supplemental material the individual latencies and amplitudes of the N100 and P180 at electrode Cz are shown.

\section{Statistical analysis}

An independent one-sided $t$-test was used to compare the RMT between patients and healthy subjects, with the hypothesis that the RMT is higher in patients because of AED use. When the RMT was higher than $100 \%$ of the stimulator output, we assumed that the RMT had a value of $101 \%$.

To compare the TEP waveform between healthy subjects and epilepsy patients, we used multiple independent $t$-tests at the electrode level. To correct for multiple comparisons, a cluster-based permutation analysis (Maris and Oostenveld 2007) was applied, as implemented in FieldTrip (http://fieldtrip.fcdonders.nl/). In short, an independent $t$-test comparing the TEPs from healthy subjects and epilepsy patients was performed for each time sample and each EEG electrode. Only $t$-values with a $p$-value $<0.01$ were considered for clustering. Clustering of $t$-values was based on adjacent time bins and neighboring electrodes. Within each found cluster, the $t$ values of the included electrodes were summed, and these summed $t$-values were used for statistical comparison. A permutation test was performed, meaning that all data was randomly assigned to the two different groups (healthy or patient) and the statistical test was performed again for 1500 times. These permutation results are then combined to form a distribution of summed clusters $t$-values. Clusters in the original data set were considered to be significant if less than $2.5 \%$ of the permutations in the distribution had a cluster-level statistic larger than the statistic in the original data set, i.e. with a $p$-value $<0.025$. We performed this analysis for the whole time-period (0-300 ms), but also using specific time regions of interest corresponding to the TEP components (20-35 ms - P30; 35-60 ms - N45; 60-80 ms - P60; 85-140 ms - N100; 150-230 ms - P180). For the specific time regions of interest we used a Bonferroni-corrected $p$-value of 0.005 .

We correlated the amplitude of the P30, N100 and P180 at electrode Cz with the RMT using the Pearson correlation coefficient, both in healthy subjects and epilepsy patients. 


\section{Results}

There were no adverse events during this study. All healthy subjects and epilepsy patients tolerated the TMS protocol well. Only one patient (patient 5) showed epileptiform abnormalities (spike-wave discharges) on the resting EEG, these discharges did not occur more frequently during or after TMS. None of the patients experienced any symptoms or signs associated with their seizures during the TMS protocol or EEG recordings.

\begin{tabular}{|c|c|c|c|c|}
\hline \multirow[b]{2}{*}{ \# } & \multicolumn{2}{|c|}{ Healthy subjects } & \multicolumn{2}{|c|}{ Epilepsy patients } \\
\hline & MT left & MT right & MT left & MT right \\
\hline 1 & 87 & 78 & $>100$ & 97 \\
\hline 2 & 65 & 62 & 100 & 70 \\
\hline 3 & 80 & 65 & 74 & 93 \\
\hline 4 & 70 & 67 & 87 & $>100$ \\
\hline 5 & 70 & 82 & $>100$ & $>100$ \\
\hline 6 & 86 & 87 & $>100$ & 90 \\
\hline 7 & 65 & 61 & 80 & 80 \\
\hline 8 & 97 & 97 & 93 & 83 \\
\hline 9 & 80 & 85 & 73 & 88 \\
\hline 10 & 94 & 75 & 79 & 80 \\
\hline 11 & 88 & 91 & 80 & 72 \\
\hline 12 & 91 & 95 & 94 & 71 \\
\hline 13 & 93 & $>100$ & 87 & 78 \\
\hline 14 & 74 & 70 & & \\
\hline 15 & 77 & 76 & & \\
\hline 16 & 84 & 73 & & \\
\hline 17 & 74 & 75 & & \\
\hline 18 & 98 & 93 & & \\
\hline $\begin{array}{l}\text { Mean } \\
\pm \text { SD }\end{array}$ & $81.8 \pm 10.7$ & $79.6 \pm 12.4$ & $88.5 \pm 10.6$ & $84.9 \pm 11.0$ \\
\hline
\end{tabular}




\section{Resting motor threshold}

The resting motor thresholds are shown in table 5.2 and figure 5.1. Both left and right RMT were higher in the patient group compared to the healthy subjects, reaching statistical significance over the left hemisphere (mean \pm SD: $88.5 \% \pm$ $10.6 \%$ vs. $81.8 \% \pm 10.7 \%, \mathrm{p}=0.048$ ). There was no significant difference when we compared the left and right RMT within each group (healthy or patient). We also found no difference in RMT between the patients using a pure channel-blocker AED $(n=9)$ and the patients using a mixed AED $(n=4)$, but it has to be noted that these subgroups are very small.

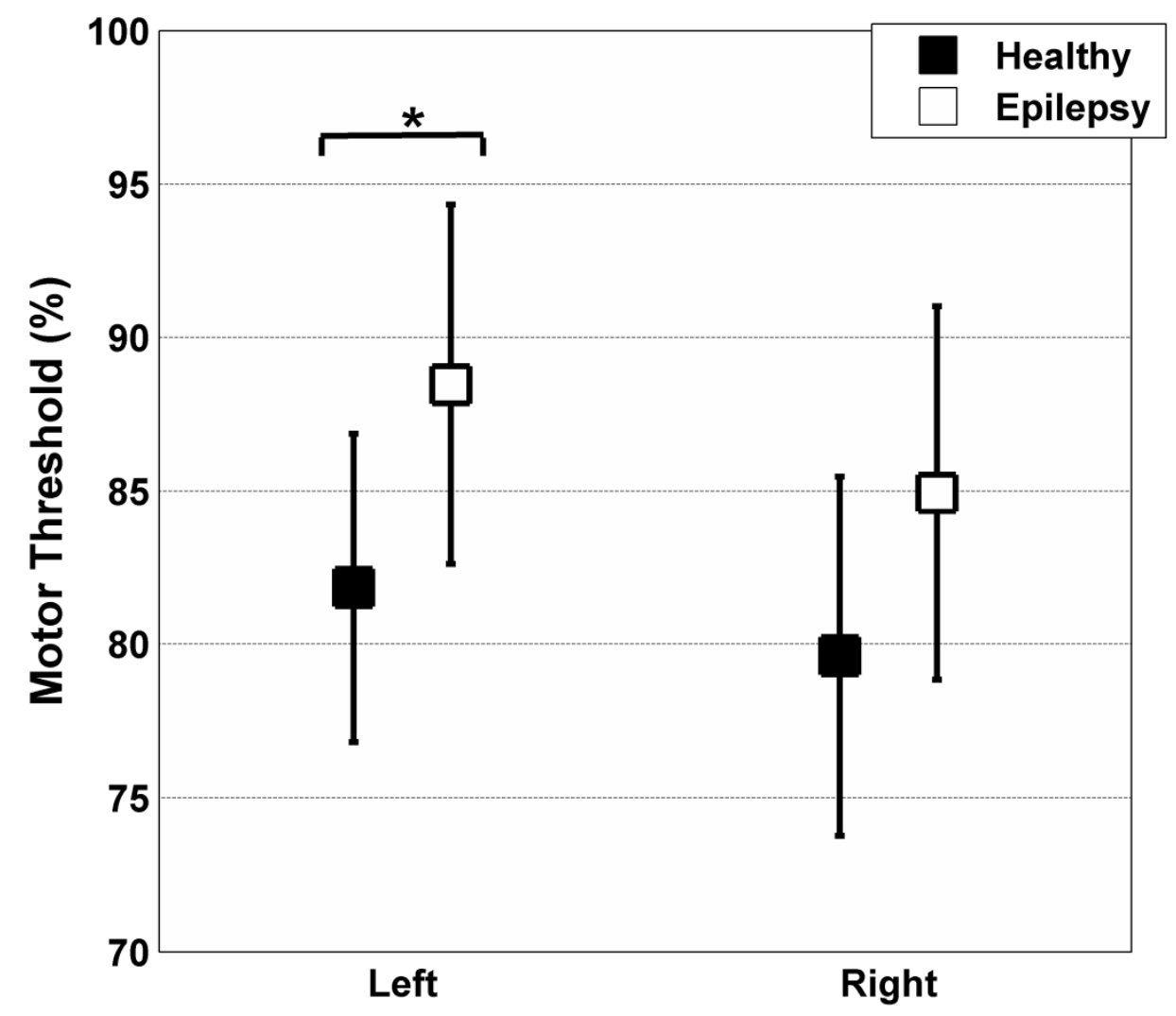

Figure 5.1. Resting motor thresholds. Mean left and right resting motor thresholds (RMT) for the healthy subjects (black square) and epilepsy patients (open square). Error bars represent the standard deviation. $*=p<0.05$. 


\section{Motor cortex TEPs}

All healthy subjects and epilepsy patients showed a characteristic TEP on electrode $\mathrm{Cz}$ when stimulating the left and right motor cortex, with components at 15, 30, 45, 60,100 and $180 \mathrm{~ms}$. In figure 5.2A and 5.2B the average TEP over subjects at electrode $\mathrm{Cz}$ and the topographical distribution of each TEP component is shown for left (5.2A) and right (5.2B) motor cortex stimulation. The distribution of the different TEP components is similar for healthy subjects and epilepsy patients, and shows the same pattern for both left and right stimulation. Activity starts in the stimulated hemisphere at parietal and frontal regions. Between 45 and $60 \mathrm{~ms}$ activity spreads to the non-stimulated hemisphere, reaching large amplitudes at centro-parietal areas at 100 and 180 ms. There was no correlation between the amplitude of the P30, N100 or P180 with RMT.

To compare the TEP between epilepsy patients and healthy subjects we performed a cluster-based permutation analysis of the TEP amplitude between 0 and $300 \mathrm{msec}$. Only clusters with a $p$-value $<0.025$ reflect significant differences in amplitude.

For left motor cortex stimulation, the N100 was larger in amplitude in epilepsy patients than in healthy subjects. There was a positive cluster consisting of 8 electrodes: P1, Pz, P2, P4, P6, POz, PO4, PO6 ( $p=0.0073)$. The largest difference in N100 amplitude was located at electrode Pz (epilepsy: $-7.00 \mu \mathrm{V}$; healthy: $-1.91 \mu \mathrm{V}$ ), see figure 5.3 (left panel). No significant clusters were found for the other TEP components.

For right motor cortex stimulation, the P180 was larger in amplitude in epilepsy patients than in healthy subjects. There was a negative cluster consisting of 6 electrodes: CP3, CP1, CPz, P3, P1, Pz ( $p=0.006)$. The largest difference in P180 amplitude was located at electrode $\mathrm{CPz}$ (epilepsy: $4.96 \mu \mathrm{V}$; healthy: $3.16 \mu \mathrm{V}$ ), see figure 5.3 (right panel). No significant clusters were found for the other TEP components.

The analysis using specific time regions of interest did not reveal additional clusters. In figure 5.4 both significant clusters are shown together with the topographical map of TMS induced activity at the N100 and P180 latency. 


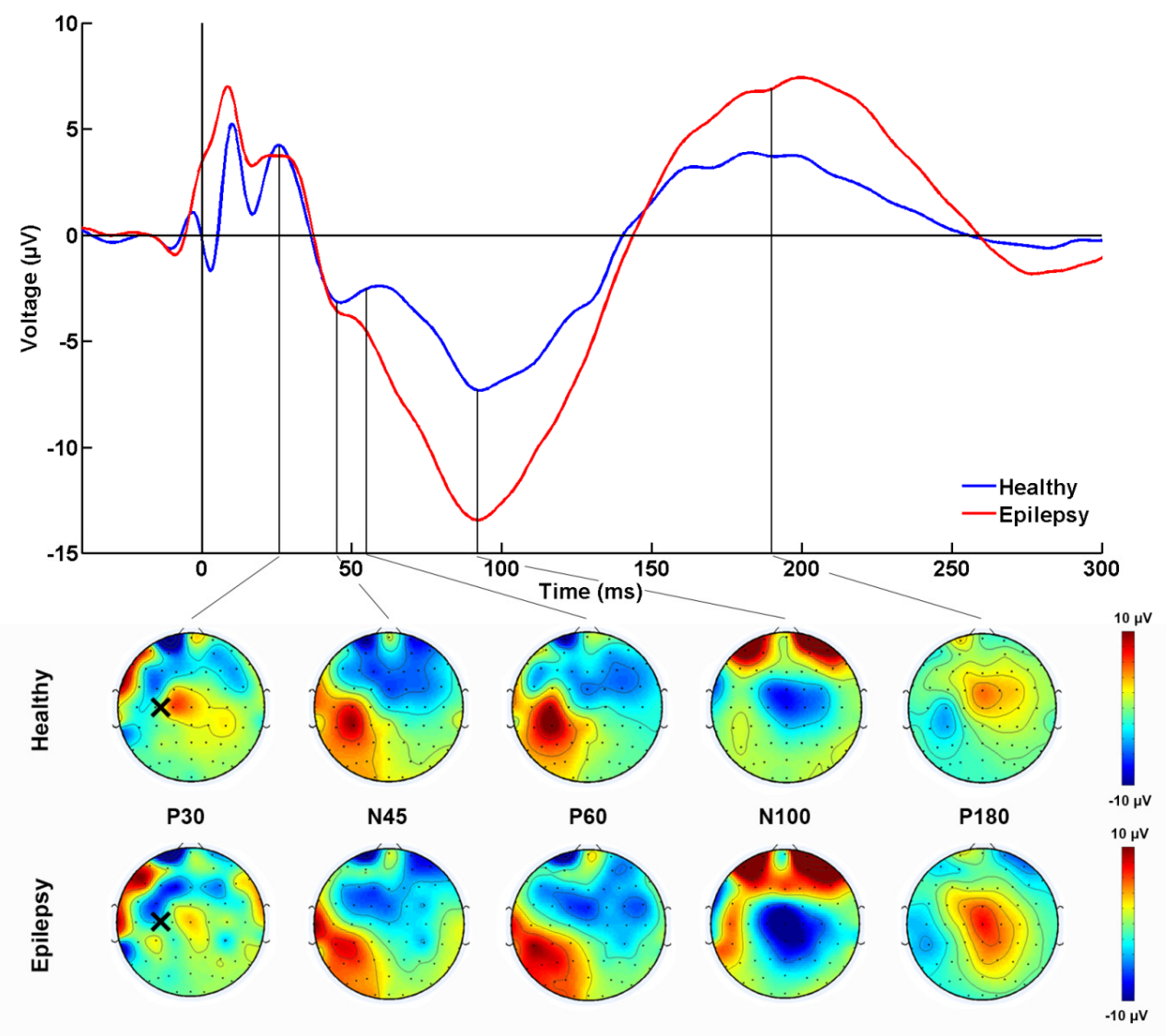

Figure 5.2A. Average TMS evoked potential at $\mathrm{Cz}$ and topographical distribution. TMS evoked potential measured at electrode $\mathrm{Cz}$ for left motor cortex stimulation for healthy subjects (blue) and epilepsy patients (red). The response is an average over all subjects. The cross represents the stimulus location. The topographical distribution of characteristic components P30, N45, P60, N100 and P180 are shown, corresponding to the time point where the component amplitude was the largest in the healthy subjects. 


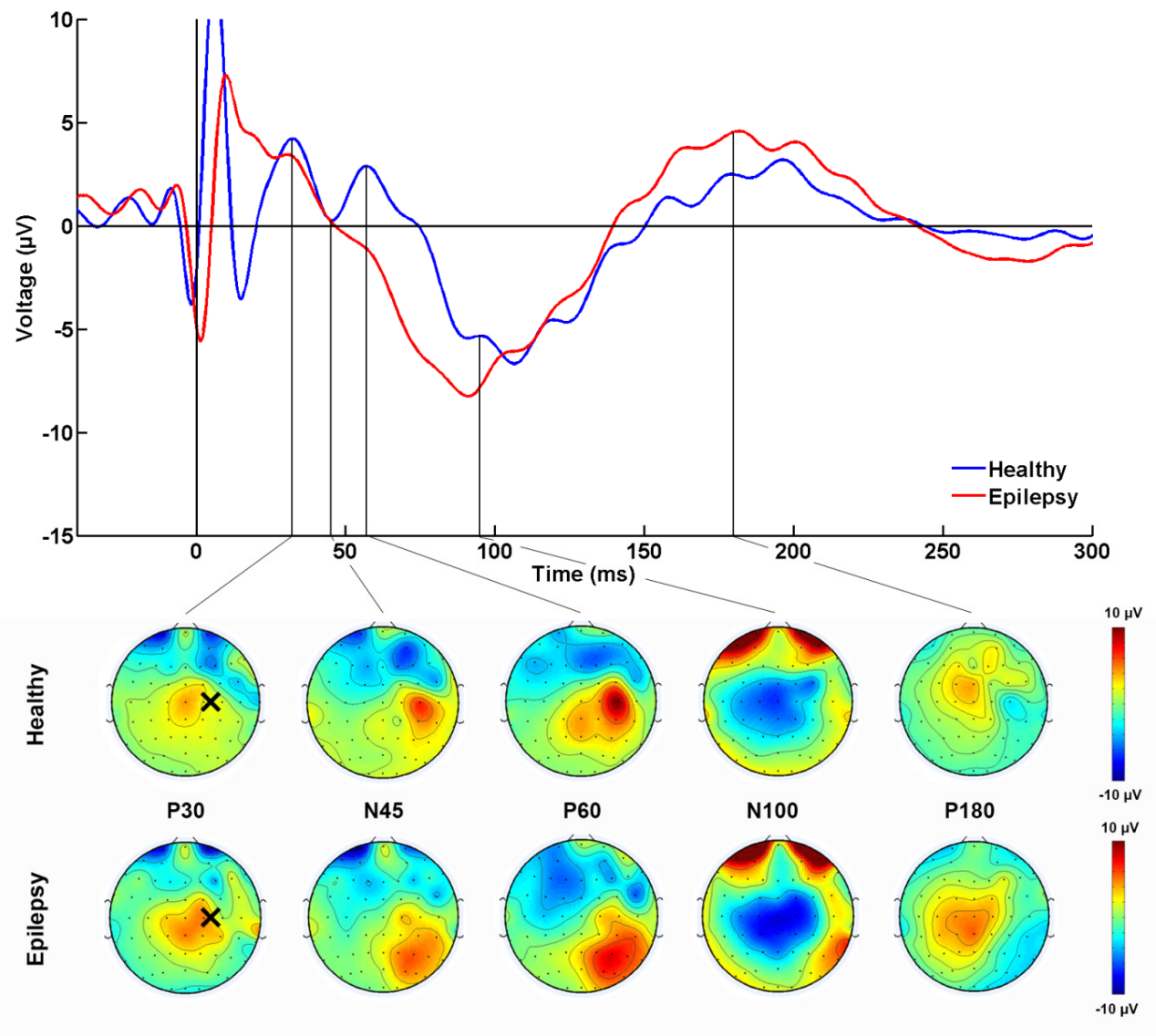

Figure 5.2B. Average TMS evoked potential at $\mathrm{Cz}$ and topographical distribution. TMS evoked potential measured at electrode $\mathrm{Cz}$ for right motor cortex stimulation for healthy subjects (blue) and epilepsy patients (red). The response is an average over all subjects. The cross represents the stimulus location. The topographical distribution of characteristic components P30, N45, P60, N100 and P180 are shown, corresponding to the time point where the component amplitude was the largest in the healthy subjects. 

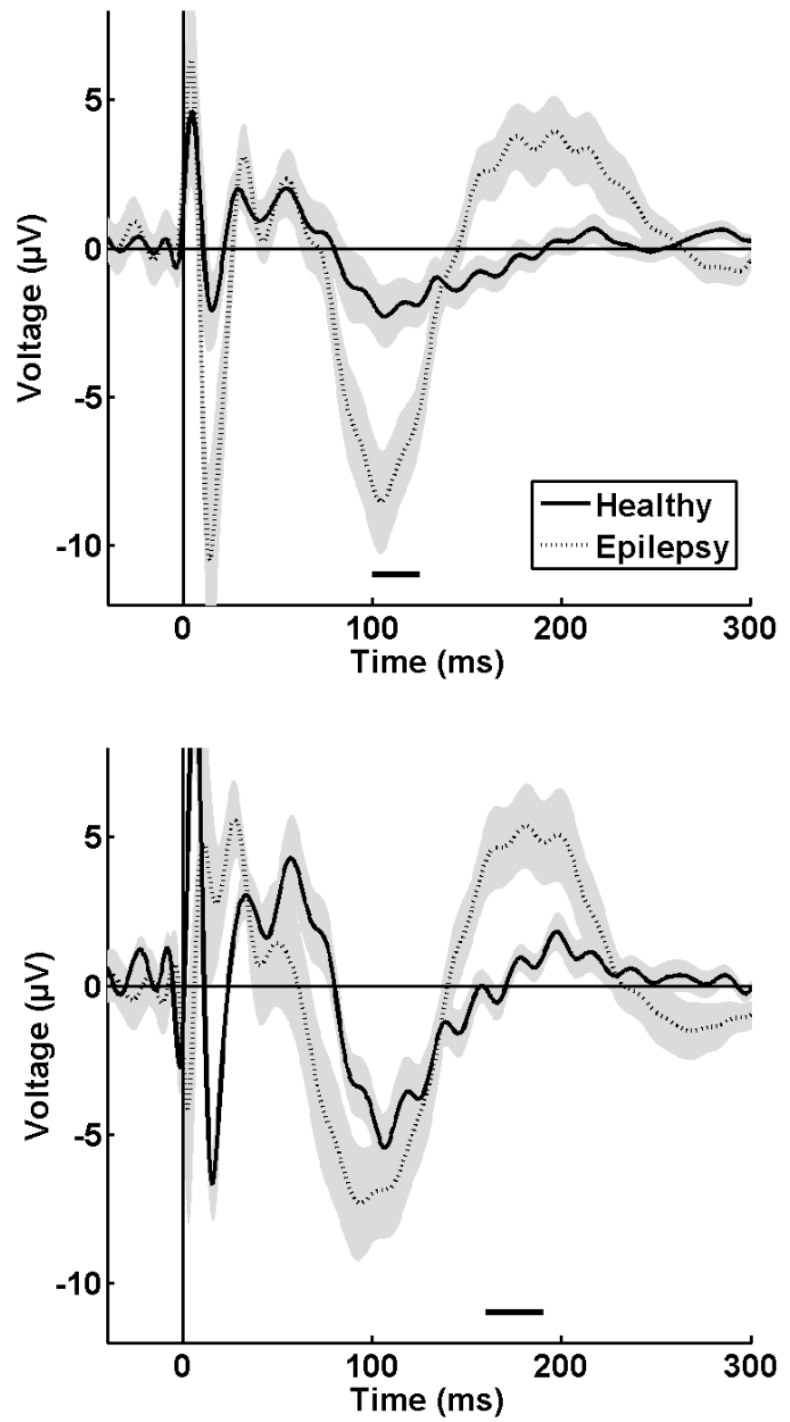

Figure 5.3. Differences in TEP components between epilepsy patients and healthy subjects. The TEP for healthy subjects (solid line) and epilepsy patients (dotted line) is shown for MCL stimulation (left) and MCR stimulation (right). Black bars underneath represent significant differences between epilepsy patients and healthy subjects. Grey areas reflect the standard error. The electrode with the largest difference between patients and healthy subjects is shown (Pz for MCL stimulation and $\mathrm{CPz}$ for MCR stimulation). 

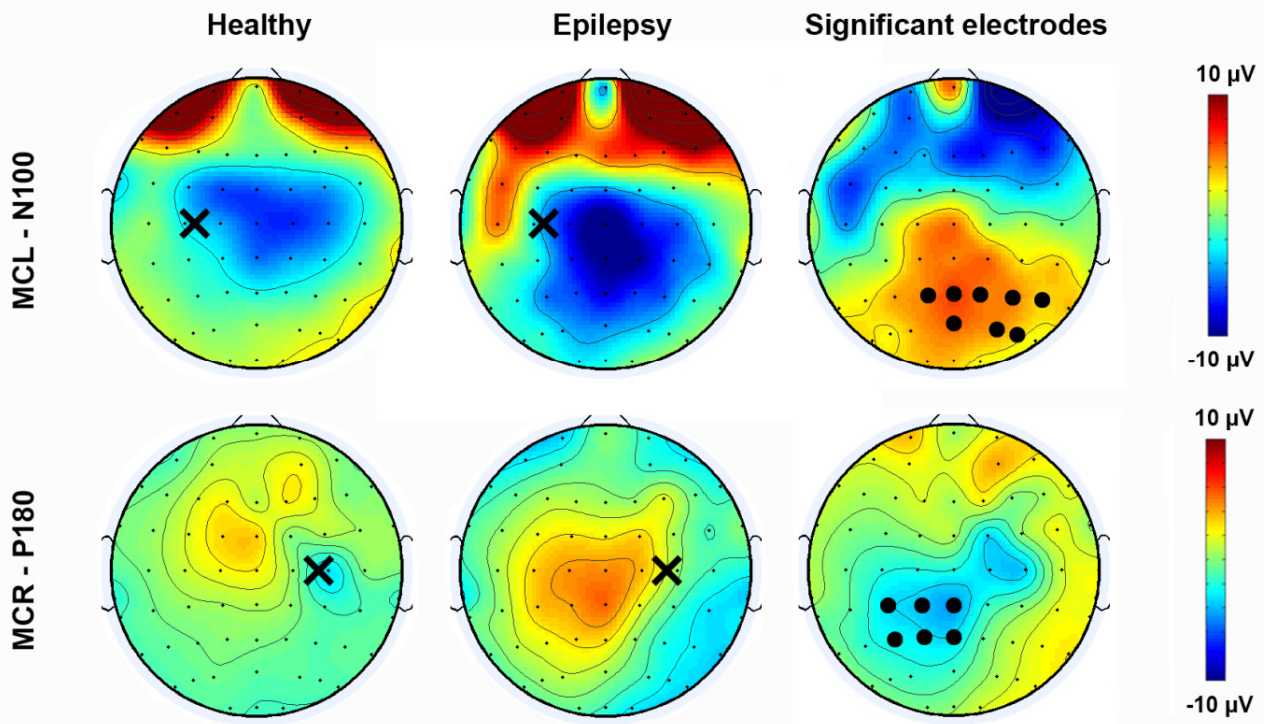

Figure 5.4. Topographical plots for TEP components N100 and P180. Topographical distribution of TEP amplitude N100 after stimulating the left motor cortex (top) and P180 after stimulating the right motor cortex (bottom) for healthy subjects (left column) and epilepsy patients (middle column). The cross represents the stimulus location. In the right column the difference between the TEP distribution is shown (healthy minus epilepsy). Black dots represent channels that show a significant difference between both groups.

\section{Discussion}

In this pilot study we stimulated the left and right motor cortex in both healthy subjects and epilepsy patients, and compared the resting motor threshold and amplitudes of the TMS evoked potential between these two groups.

We found a significantly higher left RMT in patients with epilepsy using AEDs compared to healthy controls. There were four patients and one healthy subject in whom we could not measure the RMT, because it was higher than our maximal stimulator output. Besides natural variations in RMT, this could be due to the increased distance between coil and head surface when using an EEG cap and a layer of foam, the limited maximum stimulator output ( 1.5 tesla), and of course the use of AEDs in the patient group. In these subjects we assumed an RMT of 101\%, which probably underestimates the 'true' RMT in these subjects. Previous studies reported conflicting results, ranging from a normal to an increased RMT in treated epilepsy patients (Badawy et al. 2012; Tassinari et al. 2003). However, previous findings, as 
well as the results in our patient group, are most likely influenced by the use of AEDs. Channel-blocker AEDs are known to increase the RMT, while mixed GABAergic/channel-blocker AEDs do not, but rather affect intracortical inhibition and facilitation (Ziemann et al. 1996). In particular, Lamotrigine consistently increases RMT in healthy subjects (Boroojerdi et al. 2001; Delvendahl et al. 2013; Heidegger et al. 2010; Li et al. 2009; Tergau et al. 2003; Ziemann et al. 1996), whereas RMT changes varied for Valproic acid, showing an increase in RMT in epilepsy patients (Cantello et al. 2006), and no change in RMT in healthy subjects (Li et al. 2009; Zunhammer et al. 2010). The higher RMT in our patient group is therefore very likely to be caused by AED use. The RMT was increased for epilepsy patients compared to healthy controls, but this difference reached significance only for the left hemisphere, which was the dominant hemisphere in all subjects. Previous studies found a lower RMT in the dominant hemisphere compared to the non-dominant hemisphere (De Gennaro et al. 2004; Macdonell et al. 1991; Triggs et al. 1994). In our study, such asymmetries within the groups were not observed. Two related studies also found asymmetrical differences in RMT between epilepsy patients and healthy subjects (Cantello et al. 2000; Goyal et al. 2004). We - nor the authors of these two studies - have an explanation for these findings, but one could speculate that as the brain is not perfectly symmetrical, anatomical or functional asymmetries may be involved.

The TEP showed larger amplitudes for the late components (N100 and P180) in epilepsy patients. The N100 has been related to inhibitory circuits (Casula et al. 2014; Kičić et al. 2008; Nikulin et al. 2003): an increase in N100 reflects an increase in inhibition. A previous study in EPM1 patients showed a higher amplitude of the P30 and a lower amplitude of the N100 and P180 compared to healthy controls (Julkunen et al. 2013). The authors claim that the lower later components are related to impaired inhibition, although an earlier study by the same group showed that inhibition was higher in EPM1 patients (Danner et al. 2009). Del Felice et al found that the TEP component around $100 \mathrm{~ms}$ increases in amplitude after sleep deprivation and during sleep, and that this increase was larger in JME patients than in healthy controls (Del Felice et al. 2011). Unfortunately, they did not compare the TEP during the wake state between patients and controls. However, the authors state that the increase in amplitude during sleep suggests an increased excitability, instead of an increased inhibition as reported in earlier literature. These studies evaluated different epilepsy types, but both found changes at $100 \mathrm{~ms}$. 
It has been shown recently that GABA-ergic drugs influence the TEP, with an increased N100 after taking baclofen (GABA-B), and an increased N45 and reduced N100 after taking alprazolam, zolpidem or diazepam (GABA-A) (Premoli et al. 2014a). Although the patients in the previous studies used a GABA-ergic AED with added channel-blocker (Julkunen et al. 2013) or a single mixed GABAergic/channel-blocker AED (Del Felice et al. 2011), the authors did not discuss the possible confounding effect of AEDs. In our patient group all patients were using AEDs, and it could very well be that besides GABA-ergic drugs (4/13 patients in our study) also channel-blocker AEDs (9/13 patients in our study) affect N100 TEP amplitude. Our findings of larger N100 amplitude in the epilepsy patients could therefore be explained by increased activation of inhibitory circuits, possibly because of AED use.

The origin of the P180 is not known, although the N100-P180 complex is partly caused by auditory activation due to the coil click (Nikouline et al. 1999; ter Braack et al. 2015). The left motor threshold was significantly higher in the patient group, leading to a higher stimulation intensity for the left hemisphere. Although we used the same sound masking techniques in all subjects, we cannot exclude that the higher N100 amplitude after stimulating the left motor cortex results from this difference in stimulation intensity. However, the P180 amplitude was larger in the patient group after stimulating the right motor cortex, whereas the right RMT was not significantly higher in patients. We evaluated a possible relationship between TEP amplitudes (P30-N100-P180) and RMT, but both in healthy subjects and patients we did not find any significant correlations. Therefore, it is not likely that the increased TEP amplitudes in patients are caused by a higher RMT.

There are a number of important limitations in our study. First of all, because we did not study drug naive patients, our results are most likely influenced by the use of AEDs. AEDs influence cortical excitability (Premoli et al. 2014a; Ziemann et al. 2015), and have been shown to reduce excitability in epilepsy, reflected by an increase in MT and a decrease in MEP amplitudes in paired pulse TMS, making the responses of epilepsy patients more comparable to those measured in healthy subjects (Badawy et al. 2010). In addition, the patients used different AEDs, with different (presumed) working mechanisms, which may also have different effects on the TMS measures. 
We included a relatively small group of heterogeneous patients, including both partial and generalized epilepsy, and patients with and without structural lesions on the MRI. However, epilepsy can result from a large variety of causes, and if the resulting increase in cortical excitability is comparable between different types of epilepsy, TMS-EEG may be a very robust technique in assessing this increased excitability. Badawy et al indeed showed that the changes in excitability are similar between generalized and focal (affected hemisphere) epilepsy patients (Badawy et al. 2010).

Artifacts are a major issue in TMS-EEG recordings. We used a layer of foam between coil and head a headphone playing noise to reduce the auditory evoked potential (ter Braack et al. 2015). PCA was used to reduce the first TMS artifact, originating from the TMS pulse itself, and the second TMS artifact, most likely resulting from the activation of scalp muscles (Mutanen et al. 2013; ter Braack et al. 2013). We rejected trials containing eye blinks using an automated thresholding method, but eye blinks could have still been present in our dataset at frontal channels. However, the clusters we found are located in the parietal areas, where the influence of eye blinks is minimal. Therefore, it is very unlikely that the results from our group comparison are affected by potential frontal artifacts by eye blinks.

For future studies, there are a number of issues that need to be addressed. The TEP should be evaluated in epilepsy patients before and after they start taking medication, to study excitability in drug naive patients and further explore how AEDs influence the TEP amplitudes and/or latencies in epilepsy. Additional insight may also be obtained by evaluation of the effect of different types of AEDs on the TEP in healthy subjects.

The TEP has mostly been measured using a single pulse TMS protocol, but pairedpulse TMS (ppTMS) is a promising technique to evaluate excitability as well. With this relatively new technique, series of paired TMS pulses with specific interstimulus intervals are applied to the motor cortex. Recent ppTMS studies show that patients with both focal and generalized epilepsy have an increased excitability compared to healthy subjects, presumably resulting from failure of inhibitory activity (Badawy et al. 2007; Badawy et al. 2012). This increased excitability reduces, both in focal and generalized epilepsy patients, after successful AED treatment is applied (Badawy et al. 2013; Badawy et al. 2010). Therefore, it might be interesting to evaluate the TEP 
in epilepsy patients using a ppTMS protocol. The feasibility of measuring the TEP in a ppTMS protocol has been demonstrated for short interstimulus intervals ( 3 and $11 \mathrm{~ms}$ ) (Ferreri et al. 2011) as well as long intervals (100 ms) (Premoli et al. 2014b), although Badawy et al found that even larger interstimulus intervals (especially 250 ms) consistently showed the largest difference between epilepsy patients and healthy subjects.

Lastly, instead of only looking at the TEP, future studies should also evaluate if there are late responses after single or paired pulse TMS. In our study, we found differences only in the later components of the TEP. Valentin et al showed late responses (between 100 and $1000 \mathrm{~ms}$ ) in focal epilepsy patients that were not present in healthy subjects (Valentin et al. 2008). A recent study investigated TMS-EEG in patients with epilepsy from periventricular nodular heterotopias (Shafi et al. 2015). In these patients there was an increase in late TMS induced activity $(225-700 \mathrm{~ms})$. In both studies, other stimulation sites besides the motor cortex were used, which may provide complementary information about the cortical excitability. The prolonged responses could be caused by ongoing oscillatory activity. An alternative explanation could be that multiple excitatory and inhibitory networks become active after TMS. Only at longer latencies, as the abnormal responses add up, the imbalance can be made visible with TMS-EEG. In addition to a cluster based permutation analysis, the latencies of the TEP components could have been evaluated as well. Perhaps the time course of the activity spread can differentiate between controls and epilepsy patients. In the current study, this was not explored.

In conclusion, we have shown that it is safe and feasible to apply TMS-EEG in epilepsy patients. TMS evoked potentials can be used as a relatively straight-forward method to evaluate the brain's response to single pulse TMS. In this pilot study, we found a significant higher left-sided RMT in epilepsy patients using anti-epileptic drugs compared to healthy subjects. Epilepsy patients also showed higher N100 and P180 TEP amplitudes than healthy subjects when stimulating the motor cortex. These findings suggest that TMS-EEG is a promising technique to assess the cortical excitability in epilepsy patients, although more studies are needed to evaluate the clinical relevance in epilepsy research. 


\section{Acknowledgements}

The authors wish to thank Inês Silva Santos for her assistance during the measurements, Carin Eertman, EEG technician, for her assistance during the measurements and for analyzing the resting EEG data, and the neurologists of the Medisch Spectrum Twente for their collaboration in this study.

\section{Supplemental material}

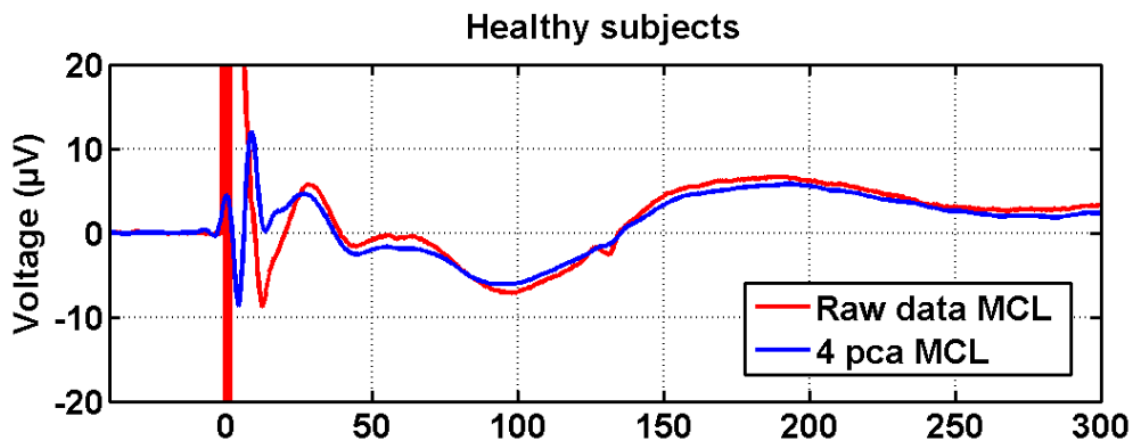

Epilepsy patients

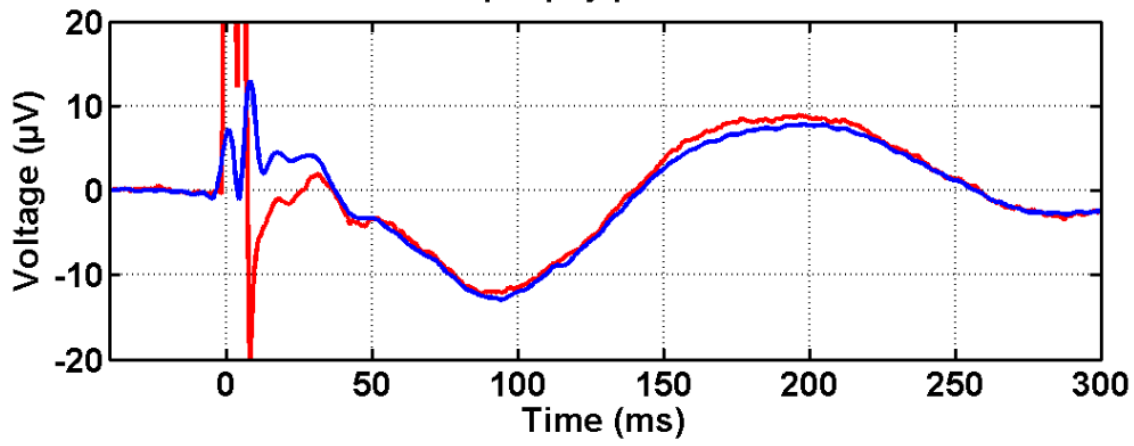

Figure S1. PCA correction after left motor cortex TMS. TMS evoked potential measured at electrode $\mathrm{Cz}$ for left motor cortex stimulation for healthy subjects (top) and epilepsy patients (bottom). The response is averaged over all subjects. Red: raw data, blue: data after removal of 4 PCA components. 


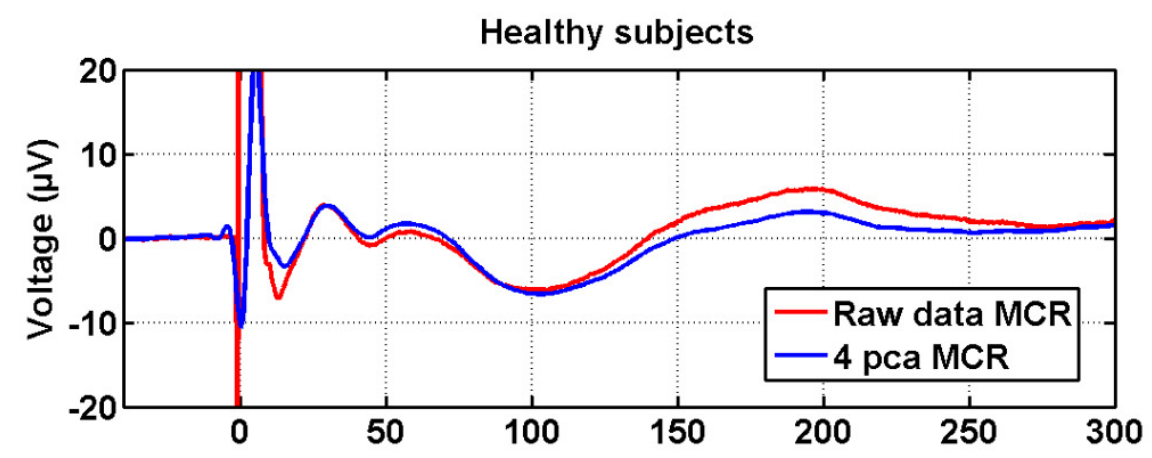

Epilepsy patients

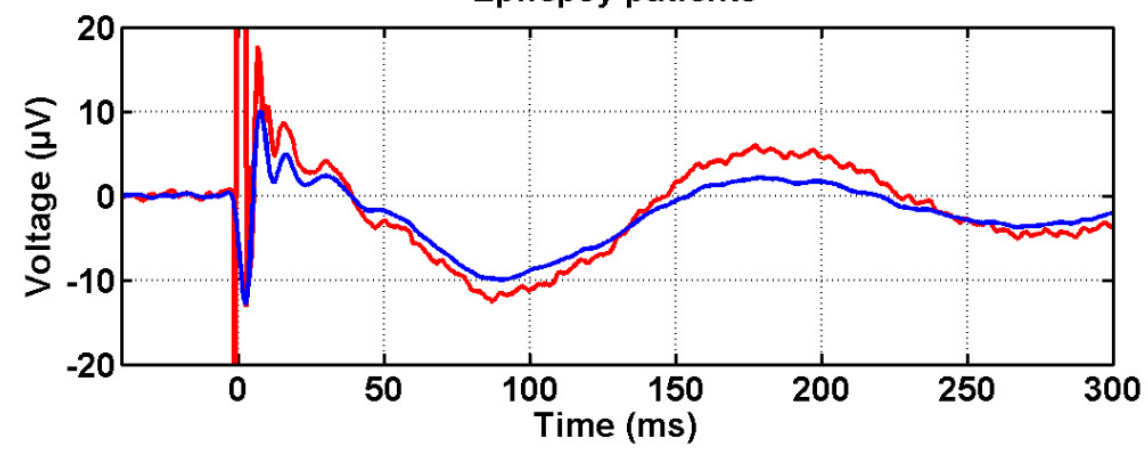

Figure S2. PCA correction after right motor cortex TMS. TMS evoked potential measured at electrode $\mathrm{Cz}$ for right motor cortex stimulation for healthy subjects (top) and epilepsy patients (bottom). The response is averaged over all subjects. Red: raw data, blue: data after removal of 4 PCA components. 

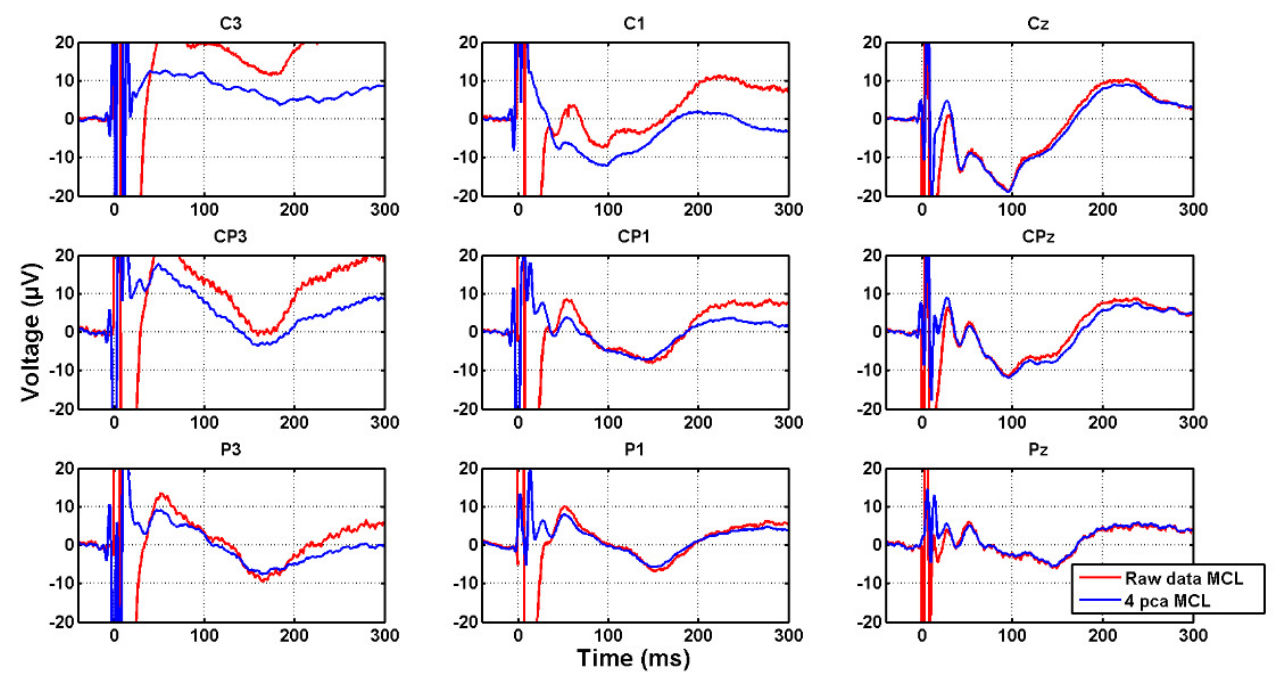

Figure S3. PCA correction for a single healthy subject. TMS evoked potential measured at 9 electrodes for a single healthy subject (subject 13) after left motor cortex stimulation. Red: raw data, blue: data after removal of 4 PCA components.
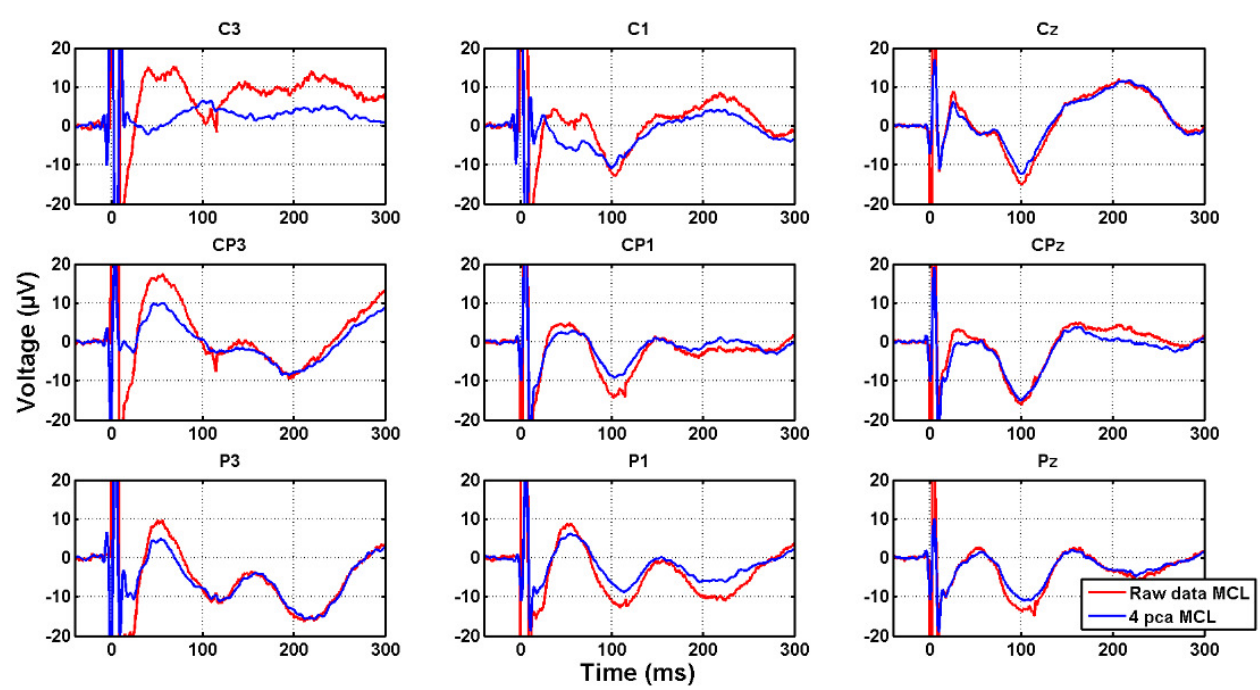

Figure S4. PCA correction for a single epilepsy patient. TMS evoked potential measured at 9 electrodes for a single epilepsy patient (patient 11) after left motor cortex stimulation. Red: raw data, blue: data after removal of 4 PCA components. 

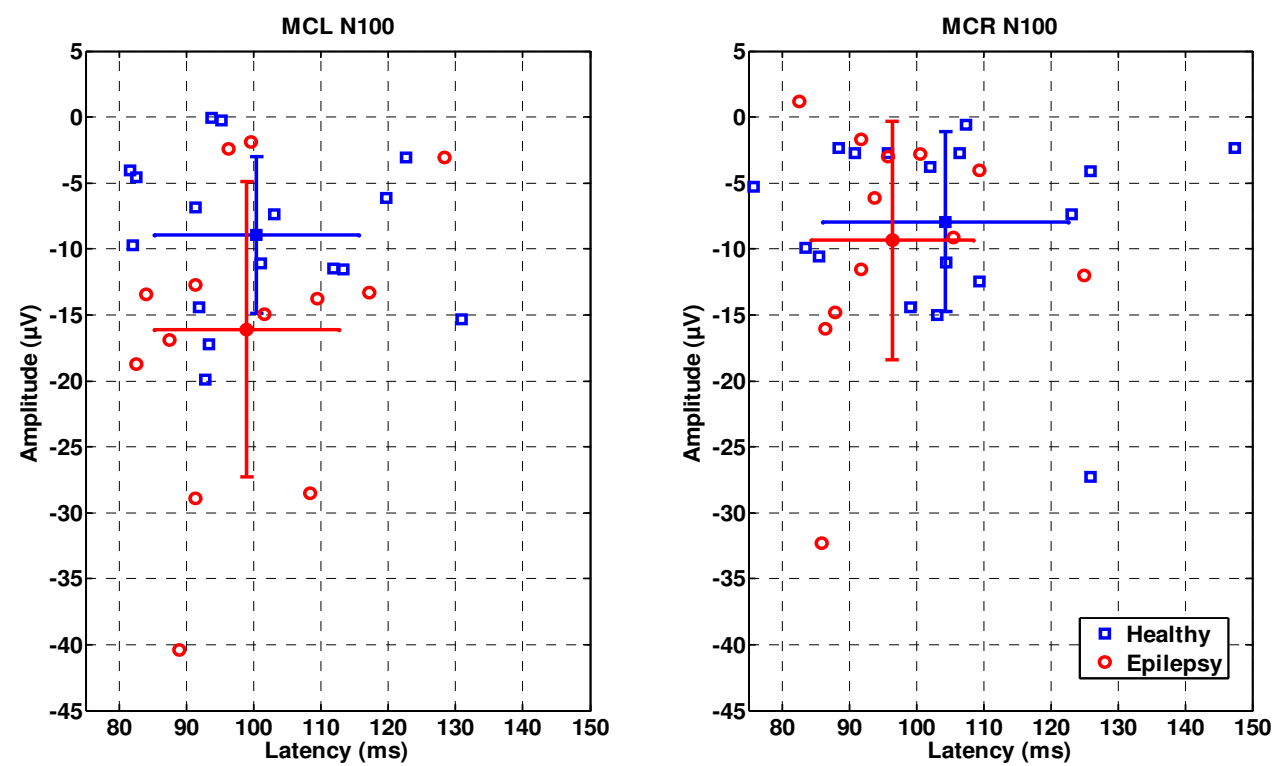

Figure S5. N100 latencies and amplitudes for Motor Cortex Left (left panel) and Motor Cortex Right (right panel) for individual healthy subjects (blue) and epilepsy patients (red) at electrode Cz. Error bars indicate the standard deviation.
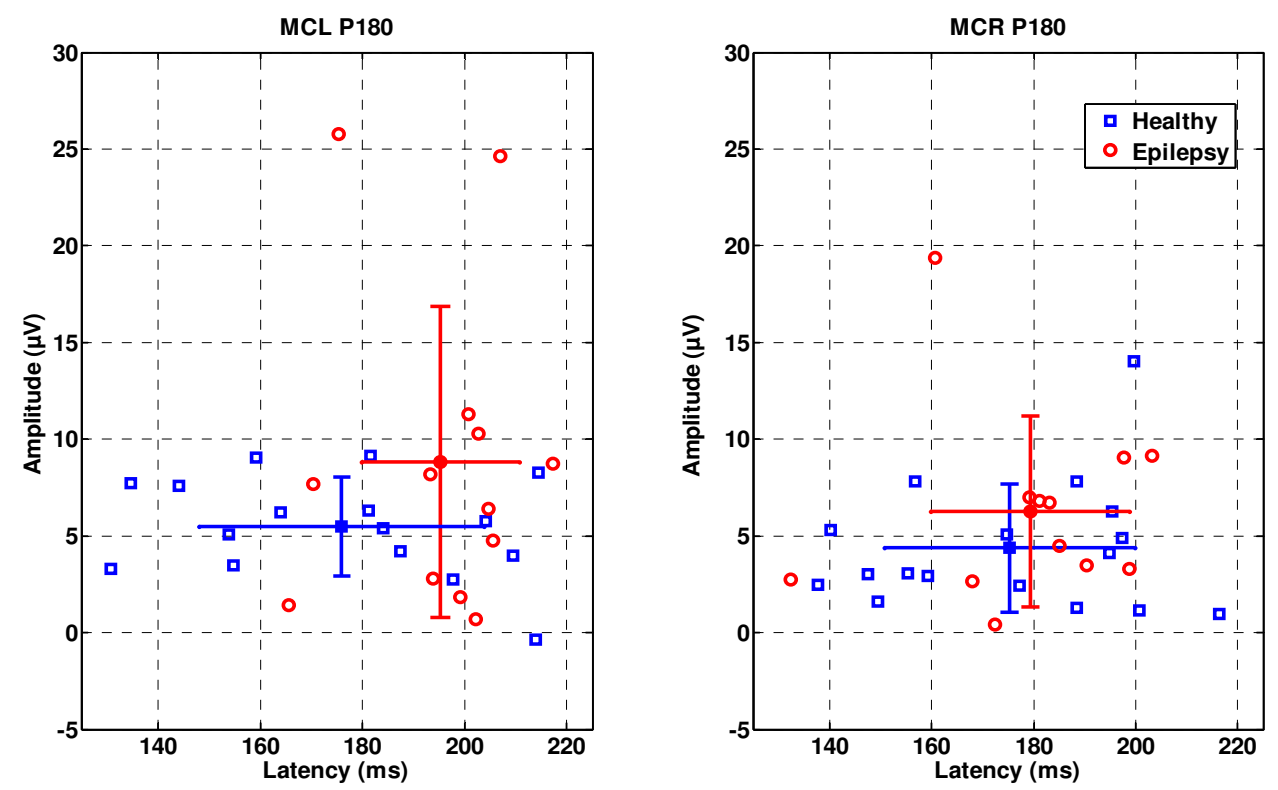

Figure S6. P180 latencies and amplitudes for Motor Cortex Left (left panel) and Motor Cortex Right (right panel) for individual healthy subjects (blue) and epilepsy patients (red) at electrode Cz. Error bars indicate the standard deviation. 


\section{References}

Badawy RA, Curatolo JM, Newton M, Berkovic SF, Macdonell RA. "Changes in cortical excitability differentiate generalized and focal epilepsy". Ann Neurol 2007; 61: 324331

Badawy RA, Jackson GD, Berkovic SF, Macdonell RA. "Excitability and refractory epilepsy: A three-year longitudinal TMS study". Int J Neur Syst 2013; 23: 1250030

Badawy RA, Loetscher T, Macdonell RA, Brodtmann A. "Cortical excitability and neurology: insights into the pathophysiology". Functional Neurology 2012; 27: 131145

Badawy RA, Macdonell RA, Berkovic SF, Newton MR, Jackson GD. "Predicting seizure control: cortical excitability and antiepileptic medication". Ann Neurol 2010; 67: 64-73

Bauer PR, Kalitzin S, Zijlmans M, Sander JW, Visser GH. "Cortical excitability as a potential clinical marker of epilepsy: a review of the clinical application of transcranial magnetic stimulation". Int J Neural Syst 2014; 24: e1430001

Bonato C, Miniussi C, Rossini PM. "Transcranial magnetic stimulation and cortical evoked potentials: a TMS/EEG co-registration study". Clin Neurophysiol 2006; 117: 16991707

Boroojerdi B, Battaglia F, Muellbacher W, Cohen LG. "Mechanisms influencing stimulusresponse properties of the human corticospinal system". Clin Neurophysiol 2001; 112: 931-937

Cantello R, Civardi C, Cavalli A, Varrasi C, Tarletti R, Monaco F, Migliaretti G. "Cortical excitability in cryptogenic localization-related epilepsy: Interictal transcranial magnetic stimulation studies". Epilepsia 2000; 41: 694-704

Cantello R, Civardi C, Varrasi C, Vicentini R, Cecchin M, Boccagni C, Monaco F. "Excitability of the human epileptic cortex after chronic valproate: A reappraisal". Brain Research 2006; 1099: 160-166

Casula EP, Tarantino V, Basso D, Arcara G, Marino G, Toffolo GM et al. "Low-frequency rTMS inhibitory effects in the primary motor cortex: Insights from TMS-evoked potentials". Neuroimage 2014; 98: 225-232

Danner N, Julkunen P, Khyuppenen J, Hukkanen T, Könönen M, Säisänen L et al. "Altered cortical inhibition in Unverricht-Lundborg type progressive myoclonus epilepsy (EPM1)". Epilepsy Res 2009; 85: 81-88

De Gennaro L, Cristiani R, Bertini M, Curcio G, Ferrara M, Fratello F et al. "Handedness is mainly associated with an asymmetry of corticospinal excitability and not of transcallosal inhibition". Clin Neurophysiol 2004; 115: 1305-1312

Del Felice A, Fiaschi A, Bongiovanni GL, Savazzi S, Manganotti P. "The sleep-deprived brain in normals and patients with juvenile myoclonic epilepsy: A perturbational approach to measuring cortical reactivity". Epilepsy Research 2011; 96: 123-131 
Delvendahl I, Lindemann H, Heidegger T, Normann C, Ziemann U, Mall V. "Effects of lamotrigine on human motor cortex plasticity". Clinical Neurophysiology 2013; 124: $148-153$

Esser SK, Huber R, Massimini M, Peterson MJ, Ferrarelli F, Tononi G. "A direct demonstration of cortical LTP in humans: a combined TMS/EEG study". Brain Res Bull 2006; 69: 86-94

Farzan F, Barr MS, Hoppenbrouwers SS, Fitzgerald PB, Chen R, Pascual-Leone A, Daskalakis ZJ. "The EEG correlates of the TMS-induced EMG silent period in humans". Neuroimage 2013; 83: 120-134

Ferreri F, Pasqualetti P, Määttä S, Ponzo D, Ferrarelli F, Tononi G et al. "Human brain connectivity during single and paired pulse transcranial magnetic stimulation". Neuroimage 2011; 54: 90-102

Fisher RS, van Emde Boas W, Blume W, Elger C, Genton P, Lee P, Engel J. "Epileptic seizures and epilepsy: Definitions proposed by the International League Against Epilepsy (ILAE) and the International Bureau for Epilepsy (IBE)". Epilepsia 2005; 46: 470-472

Goyal V, Bhatia M, Behari M. "Increased depressant effect of phenytoin sodium as compared to carbamazepine on cortical excitability: a transcranial magnetic evaluation". Neurology India 2004; 52: 224-227

Heidegger T, Krakow K, Ziemann U. "Effects of antiepileptic drugs on associative LTP-like plasticity in human motor cortex". Eur J Neurosci 2010; 32: 1215-1222

Ilmoniemi RJ, Kičić D. "Methodology for combined TMS and EEG". Brain Topogr 2010; 22: $233-248$

Julkunen P, Säisänen L, Könönen M, Vanninen R, Kälviäinen R, Mervaala E. "TMS-EEG reveals impaired intracortical interactions and coherence in Unverricht-Lundborg type progressive myoclonus epilepsy (EPM1)". Epilepsy Research 2013; 106: $103-$ 112

Kičić D, Lioumis P, Ilmoniemi RJ, Nikulin VV. "Bilateral changes in excitability of sensorimotor cortices during unilateral movement: combined electroencephalographic and transcranial magnetic stimulation study". Neuroscience 2008; 152: 1119-1129

Kimiskidis VK, Valentin A, Kalviainen R. "Transcranial magnetic stimulation for the diagnosis and treatment of epilepsy". Curr Opin Neurol 2014; 27: 236-241

Li X, Ricci R, Large CH, Anderson B, Nahas Z, George MS. "Lamotrigine and valproic acid have different effects on motorcortical neuronal excitability". Journal of Neural Transmission 2009; 116: 423-429

Macdonell RA, Shapiro BE, Chiappa KH, L HS, Cros D, Day BJ, T SB. "Hemispheric threshold differences for motor evoked potentials produced by magnetic coil stimulation". Neurology 1991; 41: 1441-1444 
Mäki H, Ilmoniemi RJ. "The relationship between peripheral and early cortical activation induced by transcranial magnetic stimulation". Neurosci Lett 2010; 478: 24-28

Maris E, Oostenveld R. "Nonparametric statistical testing of EEG- and MEG-data". J Neurosci Methods 2007; 164: 177-190

McCormick D, Contreras D. "On the cellular and network bases of epileptic seizures". Annu Rev Physiol 2001; 63: 815-846

Mutanen T, Mäki H, Ilmoniemi RJ. "The Effect of Stimulus Parameters on TMS-EEG Muscle Artifacts". Brain Stimulation 2013; 6: 371-376

Nikouline V, Ruohonen J, Ilmoniemi RJ. "The role of the coil click in TMS assessed with simultaneous EEG". Clin Neurophysiol 1999; 110: 1325-1328

Nikulin VV, Kičić D, Kähkönen S, Ilmoniemi RJ. "Modulation of electroencephalographic responses to transcranial magnetic stimulation: evidence for changes in cortical excitability related to movement". Eur J Neurosci 2003; 18: 1206-1212

Premoli I, Castellanos N, Rivolta D, Belardinelli P, Bajo R, Zipser C et al. "TMS-EEG signatures of GABAergic neurotransmission in the human cortex". J Neurosci 2014a; 34: 5603-5612

Premoli I, Rivolta D, Espenhahn S, Castellanos N, Belardinelli P, Ziemann U, MullerDahlhaus F. "Characterization of GABAB-receptor mediated neurotransmission in the human cortex by paired-pulse TMS-EEG". Neuroimage 2014b; 103: 152-162

Rossi S, Hallett M, Rossini PM, Pascual-Leone A, of TMS Consensus Group TS. "Safety, ethical considerations, and application guidelines for the use of transcranial magnetic stimulation in clinical practice and research". Clin Neurophysiol 2009; 120: 2008-2039

Rossini PM, Barker AT, Berardelli A, Caramia MD, Caruso G, Cracco RQ et al. "Noninvasive electrical and magnetic stimulation of the brain, spinal cord and roots: basic principles and procedures for routine clinical application. Report of an IFCN committee". Electroencephalogr Clin Neurophysiol 1994; 91: 79-92

Shafi MM, Vernet M, Klooster D, Chu CJ, Boric K, Barnard ME et al. "Physiological consequences of abnormal connectivity in a developmental epilepsy". Ann Neurol 2015; 77: 487-503

Tassinari CA, Cincotta M, Zaccara G, Michelucci R. "Transcranial magnetic stimulation and epilepsy". Clin Neurophysiol 2003; 114: 777-798

ter Braack EM, de Jonge B, van Putten MJ. "Reduction of TMS Induced Artifacts in EEG Using Principal Component Analysis". IEEE Trans Neural Syst Rehabil Eng 2013; 21: 376-382

ter Braack EM, de Vos CC, van Putten MJ. "Masking the Auditory Evoked Potential in TMSEEG: A Comparison of Various Methods". Brain Topography 2015; 28: 520-528

Tergau F, Wischer S, Somal HS, Nitsche MA, Joe Mercer A, Paulus W, Steinhoff BJ. "Relationship between lamotrigine oral dose, serum level and its inhibitory effect 
on CNS: insights from transcranial magnetic stimulation". Epilepsy Research 2003; 56: 67-77

Triggs WJ, Calvanio R, Macdonell RA, Cross D, Chiappa KH. "Physiological motor asymmetry in human handedness: evidence from transcranial magnetic stimulation". Brain Research 1994; 636: 270-276

Valentin A, Arunachalam R, Mesquita-Rodrigues A, Seoane JJ, Richardson MP, Mills KR, Alarcon G. "Late EEG responses triggered by transcranial magnetic stimulation (TMS) in the evaluation of focal epilepsy". Epilepsia 2008; 49: 470-480

Verrotti A, Beccaria F, Fiori F, Montagnini A, Capovilla G. "Photosensitivity: epidemiology, genetics, clinical manifestations, assessment, and management". Epileptic Disord 2012; $14: 349-362$

Ziemann U, Lönnecker S, Steinhoff BJ, Paulus W. "Effects of antiepileptic drugs on motor cortex excitability in humans: A Transcranial magnetic stimulation study". Ann Neurol 1996; 40: 367-378

Ziemann U, Reis J, Schwenkreis P, Rosanova M, Strafella A, Badawy R, Muller-Dahlhaus F. "TMS and drugs revisited 2014". Clin Neurophysiol 2015; 126: 1847-1868

Zunhammer M, Langguth B, Landgrebe M, Frank E, Müller S, Burger J et al. "Modulation of human motor cortex excitability by valproate". Psychopharmacology 2010; 215: 277-280 


\section{Chapter 6}

General Discussion 
Epilepsy is characterized by the occurrence of seizures, and results from an imbalance in excitation and inhibition (McCormick and Contreras 2001). A patient is diagnosed with epilepsy when 1) there have been two unprovoked seizures with an interval of $>24$ hours; or when 2) there has been one seizure but there is an increased risk of more seizures; or when 3 ) the patient's history already indicates a specific epilepsy syndrome (Fisher et al. 2014). Approximately $10 \%$ of the population experiences one or more seizures during their lifetime when single seizures are also included (Annegers et al. 1995). There is often uncertainty regarding epilepsy diagnosis, especially when only one seizure occurred without abnormalities in the EEG or MRI scan. When epilepsy has been diagnosed, it can take up to several months to achieve seizure freedom with AEDs or to conclude that the patient has refractory epilepsy. When we do not want to use seizure recurrence as guidance to epilepsy diagnosis or evaluation of AED success, we need another method to assess whether there (still) is an increased risk of seizures. TMS is considered to be a candidate tool for a new biomarker in epilepsy by assessing the excitability of the cortex (Bauer et al. 2014; Chen et al. 2008; Engel 2008; Kimiskidis 2016; Manganotti and del Felice 2013).

In this thesis we used a dedicated robot-navigated TMS-EEG setup to measure the TEP after stimulating the motor cortex. We investigated PCA (chapter 2) and noise masking (chapter 3) to remove and prevent artifacts. We evaluated the RMT and TEP in healthy subjects during the day (chapter 4). Finally, we compared the TEP between healthy subjects and epilepsy patients (chapter 5). In this final chapter the results from the previous chapters will be discussed and we will give possible directions for future research.

\section{TMS-EEG and artifacts}

Artifacts in TMS-EEG measurements are a major challenge, and need to be addressed before TEP analysis can be performed. Besides common EEG artifacts such as $50 \mathrm{~Hz}$ noise and eye blinks, there are multiple TMS related artifacts that can be present in a TMS-EEG recording, having both technical and physiological causes (Ilmoniemi et al. 2015; Rogasch et al. 2017). The artifact of the TMS pulse itself can be considered as a minor issue: with a dedicated amplifier this artifact is very large, but it only lasts for 1-2 ms. Therefore it does not interfere with the TEP. To eliminate this artifact, simple interpolation can be used, where the artifact is removed from the single trials and replaced by a smooth line. 
However, physiological artifacts induced by the TMS pulse remain a major concern for TEP analysis, since these artifacts overlap with the TEP. First, TMS can activate scalp muscles, resulting in a large bipolar artifact in the EEG (Mutanen et al. 2013; Rogasch et al. 2013). Second, the TMS pulse induces an auditory evoked potential (AEP), because the pulse is accompanied by a clicking sound. With higher TMS intensities the clicking sound is also louder, and the amplitude of the AEP components increases. Third, TMS stimulates sensory nerves located underneath the coil, resulting in a somatosensory evoked potential (SSEP) in the EEG. The amplitude of the SSEP is likely dependent on the TMS stimulation intensity as well. There are two ways of dealing with these physiological artifacts: removing them from the recording afterwards using signal processing techniques, or preventing (reducing) the artifact by changing aspects of the TMS protocol.

\section{Artifact removal: TMS pulse artifact and muscle artifact}

At present, several artifact rejection techniques have been used in TMS-EEG. These consist of Independent Component Analysis (ICA) (Hamidi et al. 2010; Iwahashi et al. 2008; Korhonen et al. 2011; Rogasch et al. 2014), Principal Component Analysis (PCA) (Hernandez-Pavon et al. 2012; Mäki and Ilmoniemi 2010; Rogasch et al. 2017) and other techniques (Casula et al. 2017; Litvak et al. 2007; Morbidi et al. 2007). To assist researchers, attempts have been made to create dedicated artifact rejection toolboxes (Atluri et al. 2016; Rogasch et al. 2017; Wu et al. 2018).

We have shown that with PCA the TMS pulse artifact and the muscle artifact can be removed simultaneously (chapter 2). These two artifacts have very large amplitudes compared to neural data, and exactly this characteristic makes PCA very suitable for removing them. An advantage is that it allows for semi-automatic component removal, because the components are automatically ordered based on their variance. However, the method needs to be further optimized. We noticed that the (early) TEP components were also gradually more influenced after removing increasing numbers of PCA components. This can occur when neural components have the same direction as the artifactual components, since PCA is designed to separate the data into components that lie orthogonal to each other. Instead of removing the whole principal component, it can also only be removed for the duration of the artifacts, i.e. from TMS pulse to around $50 \mathrm{~ms}$, thereby preventing distortion of the later TEP peaks (Hernandez-Pavon et al. 2012). It may be better to decide on the number of components to be removed on a single subject level, and possibly by also looking at 
the topographies of the components. The drawback is that this makes it subjective and labor-intensive. PCA may also be combined with ICA, so first the largest artifacts can be reduced with PCA, after which ICA is used to remove smaller artifacts and also other disturbances, such as eye blinks (Hernandez-Pavon et al. 2012; Korhonen et al. 2011; Rogasch et al. 2017).

Since artifact rejection in TMS-EEG is critical, it is of great importance to compare the different techniques using a dataset containing recordings with different types of artifacts of varying magnitude. Offering such a dataset publicly online may facilitate this process. Ideally, the technique, or combination of techniques, should largely reduce the artifacts with minimal influence on the TEP components; it should perform equally well for recordings with smaller or larger artifacts; and it should preferably operate without subjective input from the user. Artifact rejection is most important when we are interested in the early components of the TEP, while later components (N100 and P180) are not corrupted by artifacts. Therefore the need for complex artifact rejection techniques can be questioned when for example the N100 is the most sensitive and/or robust as a biomarker for excitability.

\section{Artifact prevention: AEP, muscle artifact and SSEP}

Preventing artifacts is better than removing them from the data afterwards. We have shown that without any precautions the AEP is a substantial contributor to the TEP, but it can be (largely) prevented by using noise masking techniques (chapter 3). Using only white noise or noise adapted to resemble the coil click does significantly lower the amplitude of the AEP. However, to minimize the AEP a layer of foam placed between the coil and head is essential. A minor drawback of using foam is that it increases the distance between coil and head, thereby increasing the RMT. This so-called noise masking is now standard practice in TMS-EEG research.

Muscle activation artifacts can be reduced by choosing a different stimulation location (where there are no scalp muscles), lowering the stimulation intensity, or rotating/tilting the coil (Mutanen et al. 2013). Because it makes signal processing less complex, it may be worthwhile to investigate whether changing these stimulation parameters result in TEPs that are still able to discriminate between different subject groups.

We have not addressed the potential contribution of the SSEP to the TEP in this thesis. To evaluate the SSEP, two different experimental setups may be used. First, 
the skin underneath the coil may be anesthetized to prevent activation of the sensory nerves. This way, the SSEP is eliminated from the TEP and the relative contribution can be estimated. Second, an experimental protocol can be used where the sensation of TMS is imitated without the TMS pulse activating the cortex. Attempts have been made placing an electrical stimulator underneath a sham TMS coil, but it is unclear whether this gives a similar feeling as the TMS pulse (Conde et al. 2017). Even if we are able to define the contribution of the SSEP to the TEP, it would be difficult to prevent the SSEP in practice.

\section{TMS-EEG - variation of the TEP}

There are three types of variation that are of importance when evaluating TMS-EEG as a biomarker in epilepsy: 1) the inter-individual variation of the TEP; 2) the intraindividual variation of the TEP; and 3) the variation of the TEP due to small changes in the measurement protocol.

\section{Inter-individual variation}

For the TEP to be of diagnostic use there should be a detectable and reliable difference between healthy subjects and patients with epilepsy. In addition, there should be a difference between epilepsy patients and patients with a single seizure who do not have epilepsy. Both these differences have to be larger than the variation within one of these subject groups. The inter-individual variation of the TEP is very large (see for example chapter 4). Most, but not all, subjects show characteristic TEP components, but the amplitudes and latencies show a large variability between subjects. For assessing the potential diagnostic use, studies should include larger number of subjects in order to establish reference values.

\section{Intra-individual variation}

When the TEP is to be used to evaluate AED success, the difference with healthy subjects is less relevant. AEDs have an effect on excitability in general (Ziemann et al. 2015), which has also been demonstrated for lamotrigine and levetiracetam with TMS-EEG (Premoli et al. 2017). Although the exact mechanisms of AEDs are still not completely known, and the effects of AEDs on the TEP need to be further explored, a change in the amplitude of TEP components might be related to AED success or failure. In this case the variation of the TEP on an individual level is important: the change in TEP after AEDs should be larger than the normal intraindividual variation. 
In chapter 4 we showed that even though there is a large variation between subjects, the TEP is largely reproducible during the day for individual subjects. The TEP is also very constant within one measurement session and when repeating the measurement after one week (Kerwin et al. 2018; Lioumis et al. 2009). Combining our own data with these two studies suggests that the normal intra-individual variation of the TEP at different time points is low and that the TEP can be used to compare different sessions in a single subject. Therefore, the TEP seems to be wellsuited to evaluate the effect of starting or adding AEDs. However, drug induced changes of the TEP are not consistent for healthy subjects (Premoli et al. 2017; Premoli et al. 2014); and AED induced changes in LICI could not predict AED success on single-subject level (Badawy et al. 2010). It appears that the effect of AEDs on these measures of excitability differs between subjects, and that decreased excitability as measured by TMS is not the only factor that defines seizure freedom. What is also of interest is the minimum change in outcome measure that is required to obtain a statistically significant result, also referred to as the smallest detectable change (SDC). For the TEP, the lowest SDC values are found for the N100 and P180, where an intervention must induce a change of $42 \%$ in amplitude to ensure significance (Kerwin et al. 2018).

\section{Variation due to measurement protocol}

Besides the variation between and within subjects, the TMS-EEG protocol can also induce changes in the TEP. It is yet unclear which protocol parameters induce large enough changes to warrant strict control. Research has focused mostly on the stimulated area, showing that although the TEP is significantly different after stimulating different cortical regions (Casarotto et al. 2010; Kähkönen et al. 2004; Rosanova et al. 2009); the components of the TEP are very similar for all stimulation targets. This may indicate that neuronal processing of an external activation current is very generic for different cortical regions. If this is true, stimulation targets for TMS-EEG can be selected that cause the least number of artifacts and discomfort while still showing differences between healthy subjects and patients.

The need for neuronavigation when stimulating a specific target is still unclear. A recent study by our group investigated whether differences in the TEP, LICI and MEP exist when stimulating $2 \mathrm{~mm}$ or $5 \mathrm{~mm}$ from the determined hotspot, and indicates that a high accuracy in coil positioning is especially required to measure cortical excitability reliably in individual subjects. On group level no differences 
were found, and therefore we conclude that neuronavigation is of limited added value when analysis is performed on a group level (de Goede et al. submitted). However, it may still be that the amount of variation is smaller when neuronavigation is used when compared to holding the coil by hand or with a stand.

It has been shown in that the TEP is already visible with stimulation intensities as low as 60\% of RMT (Komssi et al. 2004), but these authors did not use noise masking. We found that the TEP is still visible with TMS pulses at 80-90\% RMT (unpublished data). However, the reliability of the TEP at these lower intensities still needs to be investigated. In addition to a high enough stimulation intensity, the number of trials should also be large enough to obtain a reliable response: approximately 20-30 single pulses for the MEP (Goldsworthy et al. 2016), 20-25 conditioned pulses for SICI and ICF (Chang et al. 2016) and 60-100 pulses for the TEP (Kerwin et al. 2018).

\section{TMS-EEG in epilepsy}

There have been multiple reports on TMS-EMG in drug naive epilepsy patients. Although significant differences were found for various excitability measures, with LICI as the most promising measure, TMS-EMG results have been conflicting (Bauer et al. 2018; de Goede et al. 2016). For TMS-EEG data is limited, with only a handful studies published so far. In chapter 5 we showed that there are significant differences between epilepsy patients and healthy subjects in the N100 amplitude, similar to findings by other researchers (Del Felice et al. 2011; Julkunen et al. 2013), as well as in the P180 amplitude. Changes in the N100 and P180 were also demonstrated in healthy subjects after single administrations of the AEDs levetiracetam and lamotrigine (Premoli et al. 2017). One of these two AEDs were used by 7 out of 13 epilepsy patients in our study in chapter 5 and in all seven patients from a previous study (Julkunen et al. 2013). It is therefore likely that AEDs introduced a bias in these findings in epilepsy patients, and that these results cannot be extrapolated to drug naive patients.

The N100 and P180 are of interest as possible biomarkers in TMS-EEG, because they have the largest amplitude of all components of the TEP and are not influenced by technical artifacts or muscle artifacts. In addition, of all components of the TEP, the N100 and P180 showed the highest reliability (Kerwin et al. 2018). The origin of the N100 is not yet fully understood, but evidence shows that it is linked to cortical 
GABA-B mediated inhibition (Bonnard et al. 2009; Farzan et al. 2013; Kähkönen and Wilenius 2007; Nikulin et al. 2003; Premoli et al. 2014). An increase in N100 amplitude corresponds to an increase in inhibition. The origin of the P180 remains to be elucidated, but changes in P180 in our patient study (chapter 5) and after administration of AEDs (Premoli et al. 2017) suggest that this component may also be related to inhibitory processes. It is noteworthy that the P180 amplitude was larger in our patients taking AEDs compared to healthy controls, but in healthy subjects the amplitude was reduced after administration of AEDs. Both the N100 and P180 are part of the AEP, which means that noise masking is necessary when evaluating these components.

Besides evaluation of the TEP, TMS-EEG may also be used as a method to induce epileptiform activity, similar to photic stimulation or hyperventilation. Following a previous study that showed late rhythmic activity after spTMS in 11 out of 15 epilepsy patients but not in healthy controls (Valentin et al. 2008), recently another study was published where epileptic discharges could be induced by ppTMS in 6 out of 12 refractory epilepsy patients but not in 12 well-controlled epilepsy patients or healthy controls (Kimiskidis et al. 2017). Again, both studies were biased by AED use. In our group of 13 epilepsy patients we did not see any induced epileptiform activity by spTMS (chapter 5). A large advantage of assessing induced activity is that no advanced signal processing is needed as normal EEG evaluation with visual analysis would already suffice. These findings warrant further studies in larger groups of drug naive epilepsy patients.

\section{Future perspectives}

In this thesis we defined two major challenges in epilepsy: to shorten the time needed to evaluate AED success, and to improve the diagnostic process in patients with a single seizure without EEG and MRI abnormalities. We have addressed various aspects that are important in using TMS-EEG as a biomarker, but several issues still need to be investigated.

Currently, increasingly more attention is being paid to assessing the variability and repeatability in TMS studies (Beaulieu et al. 2017; Goldsworthy et al. 2016; Guerra et al. 2017; Kerwin et al. 2018; Schmidt et al. 2015), which is needed to establish the reliability of these excitability measures. The large inter-individual variation seems to be a common aspect in both TMS-EMG and TMS-EEG that hampers not only the 
clinical use in epilepsy, but also its potential application in other diseases and neuroscience research in general. Further studies in large groups of healthy subjects are needed to assess the sources of variability. The effect of these sources should also be compared to the difference in excitability measures between healthy subjects and patients.

For evaluating AED success, studying the effects of AEDs on TMS measures in healthy subjects may assist in finding the most promising features suitable for patient studies $^{2}$. This approach will also allow quantification of the confounding effect of AEDs on excitability measures. At the same time TMS measurements should be performed in epilepsy patients before and after starting AEDs ${ }^{3}$. TMS measurements should take place at baseline and at different time points after starting and increasing AEDs. Changes in RMT were already detectable after 15 days of AED use in focal epilepsy patients (Manganotti et al. 1999), suggesting that with TMS the time to evaluate AED success may be shortened. To evaluate the predictive power of TMS measures, the changes in excitability measures need to be compared between responders and non-responders.

For the diagnostic process, reference values of TMS markers must be established in large groups of healthy subjects. Additionally, studies should focus on patients who experienced a first seizure and come to the neurology department or who are brought into the emergency department. TMS measurements have to be performed in all patients with a possible epileptic seizure ${ }^{4}$, also in patients in whom the epilepsy diagnosis can already be made based on the existing criteria (Fisher et al. 2014). Only then four different subcategories can be compared: 1) patients with epilepsy according to the ILAE criteria; 2) patients with epilepsy in whom the diagnosis is made at later stage; 3 ) patients with events that do not have epilepsy; and 4) healthy subjects.

\footnotetext{
${ }^{2}$ As currently performed at Center for Human Drug Research, study number NTR6824.

${ }^{3}$ As currently performed at Medisch Spectrum Twente, study number NTR4795.

${ }^{4}$ As currently performed at Medisch Spectrum Twente, study number NTR4793.
} 


\section{Conclusion}

This thesis focused on the first steps towards a clinical application of TMS-EEG in epilepsy. We successfully created a TMS-EEG setup and investigated the TEP in healthy subjects. Three artifacts were addressed: the magnetic artifact from the TMS pulse itself, the large bipolar artifact from scalp muscle activation, and the auditory artifact caused by the clicking sound of the TMS pulse. We showed that PCA can be used to remove the TMS pulse artifact and the muscle artifact from TMS-EEG recordings. Different types of noise masking were evaluated and we demonstrated that the AEP can be largely prevented. We assessed the variation of the TEP and found that the TEP is very constant during daytime. The inter-individual variation is however large. Lastly, we compared the TEP between healthy subjects and epilepsy patients using AEDs, and reported significant higher N100 and P180 amplitudes for the patient group. 


\section{References}

Annegers JF, Hauser WA, Lee JR-J, Rocca WA. "Incidence of acute symptomatic seizures in Rochester, Minnesota 1935-1984". Epilepsia 1995; 36: 327-333

Atluri S, Frehlich M, Mei Y, Garcia Dominguez L, Rogasch NC, Wong W et al. "TMSEEG: A MATLAB-Based Graphical User Interface for Processing Electrophysiological Signals during Transcranial Magnetic Stimulation". Front Neural Circuits 2016; 10: 78

Badawy RA, Macdonell RA, Berkovic SF, Newton MR, Jackson GD. "Predicting seizure control: cortical excitability and antiepileptic medication". Ann Neurol 2010; 67: 64-73

Bauer PR, de Goede AA, Stern WM, Pawley AD, Chowdhury FA, Helling RM et al. "Longinterval intracortical inhibition as biomarker for epilepsy: a transcranial magnetic stimulation study". Brain 2018; 141: 409-421

Bauer PR, Kalitzin S, Zijlmans M, Sander JW, Visser GH. "Cortical excitability as a potential clinical marker of epilepsy: a review of the clinical application of transcranial magnetic stimulation". Int J Neural Syst 2014; 24: e1430001

Beaulieu LD, Flamand VH, Masse-Alarie H, Schneider C. "Reliability and minimal detectable change of transcranial magnetic stimulation outcomes in healthy adults: A systematic review". Brain Stimul 2017; 10: 196-213

Bonnard M, Spieser L, Meziane HB, de Graaf JB, Pailhous J. "Prior intention can locally tune inhibitory processes in the primary motor cortex: direct evidence from combined TMS-EEG". Eur J Neurosci 2009; 30: 913-923

Casarotto S, Lauro LJR, Bellina V, Casali AG, Rosanova M, Pigorini A et al. "EEG responses to TMS are sensitive to changes in the perturbation parameters and repeatable over time". PLoS One 2010; 5: e10281

Casula EP, Bertoldo A, Tarantino V, Maiella M, Koch G, Rothwell JC et al. "TMS-evoked long-lasting artefacts: A new adaptive algorithm for EEG signal correction". Clin Neurophysiol 2017; 128: 1563-1574

Chang WH, Fried PJ, Saxena S, Jannati A, Gomes-Osman J, Kim YH, Pascual-Leone A. "Optimal number of pulses as outcome measures of neuronavigated transcranial magnetic stimulation". Clin Neurophysiol 2016; 127: 2892-2897

Chen R, Cros D, Curra A, Lazzaro VD, Lefaucheur J-P, Magistris MR et al. "The clinical diagnostic utility of transcranial magnetic stimulation: report of an IFCN committee". Clin Neurophysiol 2008; 119: 504-532

Conde V, Akopian I, Tomasevic L, Stanek K, Bergmann TO, Siebner HR. "P122 Contribution of integrated somatosensory and auditory inputs to the cortical response evoked by transcranial magnetic stimulation: A sham TMS-EEG study". Clinical Neurophysiology 2017; 128: e75-e76 
de Goede AA, Ter Braack EM, van Putten M. "Single and paired pulse transcranial magnetic stimulation in drug naive epilepsy". Clin Neurophysiol 2016; 127: 3140-3155

de Goede AA, Ter Braack EM, van Putten MJ. "Varying the coil location and orientation in single and paired pulse TMS". submitted;

Del Felice A, Fiaschi A, Bongiovanni GL, Savazzi S, Manganotti P. "The sleep-deprived brain in normals and patients with juvenile myoclonic epilepsy: A perturbational approach to measuring cortical reactivity". Epilepsy Research 2011; 96: 123-131

Engel J, Jr. "Progress in epilepsy Reducing the treatment gap and the promise of biomarkers". Curr Opin Neurol 2008; 21: 50-54

Farzan F, Barr MS, Hoppenbrouwers SS, Fitzgerald PB, Chen R, Pascual-Leone A, Daskalakis ZJ. "The EEG correlates of the TMS-induced EMG silent period in humans". Neuroimage 2013; 83: 120-134

Fisher RS, Acevedo C, Arzimanoglou A, Bogacz A, Cross JH, Elger CE et al. "ILAE official report: a practical clinical definition of epilepsy". Epilepsia 2014; 55: 475-482

Goldsworthy MR, Hordacre B, Ridding MC. "Minimum number of trials required for withinand between-session reliability of TMS measures of corticospinal excitability". Neuroscience 2016; 320: 205-209

Guerra A, Lopez-Alonso V, Cheeran B, Suppa A. "Solutions for managing variability in noninvasive brain stimulation studies". Neurosci Lett 2017;

Hamidi M, Slagter HA, Tononi G, Postle BR. "Brain responses evoked by high-frequency repetitive transcranial magnetic stimulation: an event-related potential study". Brain Stimul 2010; 3: 2-14

Hernandez-Pavon JC, Metsomaa J, Mutanen T, Stenroos M, Mäki H, Ilmoniemi RJ, Sarvas J. "Uncovering neural independent components from highly artifactual TMSevoked EEG data". Journal of Neuroscience Methods 2012; 209: 144-157

Ilmoniemi RJ, Hernandez-Pavon JC, Makela NN, Metsomaa J, Mutanen M, Stenroos M, Sarvas J. "Dealing with artifacts in TMS-evoked EEG". Conf Proc IEEE Eng Med Biol Soc 2015; 2015: 230-233

Iwahashi M, Arimatsu T, Ueno S, Iramina K. "Differences in evoked EEG by transcranial magnetic stimulation at various stimulus points on the head". Conf Proc IEEE Eng Med Biol Soc 2008; 2008: 2570-2573

Julkunen P, Säisänen L, Könönen M, Vanninen R, Kälviäinen R, Mervaala E. "TMS-EEG reveals impaired intracortical interactions and coherence in Unverricht-Lundborg type progressive myoclonus epilepsy (EPM1)". Epilepsy Research 2013; 106: 103112

Kähkönen S, Wilenius J. "Effects of alcohol on TMS-evoked N100 responses". J Neurosci Methods 2007; 166: 104-108

Kähkönen S, Wilenius J, Komssi S, Ilmoniemi RJ. "Distinct differences in cortical reactivity of motor and prefrontal cortices to magnetic stimulation". Clin Neurophysiol 2004; 115: $583-588$ 
Kerwin LJ, Keller CJ, Wu W, Narayan M, Etkin A. "Test-retest reliability of transcranial magnetic stimulation EEG evoked potentials". Brain Stimul 2018; 11: 536-544

Kimiskidis VK. "TMS coupled with EEG Biomarker of the future". Revue Neurologique 2016; 172: 123-126

Kimiskidis VK, Tsimpiris A, Ryvlin P, Kalviainen R, Koutroumanidis M, Valentin A et al. "TMS combined with EEG in genetic generalized epilepsy: A phase II diagnostic accuracy study". Clin Neurophysiol 2017; 128: 367-381

Komssi S, Kähkönen S, Ilmoniemi RJ. "The effect of stimulus intensity on brain responses evoked by transcranial magnetic stimulation". Hum Brain Mapp 2004; 21: 154-164

Korhonen RJ, Hernandez-Pavon JC, Metsomaa J, Mäki H, Ilmoniemi RJ, Sarvas J. "Removal of large muscle artifacts from transcranial magnetic stimulation-evoked EEG by independent component analysis". Med Biol Eng Comput 2011; 49: 397-407

Lioumis P, Kičić D, Savolainen P, Mäkelä JP, Kähkönen S. "Reproducibility of TMS-Evoked EEG responses". Hum Brain Mapp 2009; 30: 1387-1396

Litvak V, Komssi S, Scherg M, Hoechstetter K, Classen J, Zaaroor M et al. "Artifact correction and source analysis of early electroencephalographic responses evoked by transcranial magnetic stimulation over primary motor cortex". Neuroimage 2007; 37: $56-70$

Mäki H, Ilmoniemi RJ. "Projecting out muscle artifacts from TMS-evoked EEG". Neuroimage 2010; 54: 2706-2710

Manganotti P, Bongiovanni LG, Zanette G, Turazzini M, Fiaschi A. "Cortical excitability in patients after loading doses of lamotrigine: a study with magnetic brain stimulation". Epilepsia 1999; 40: 316-321

Manganotti P, del Felice A. "New perspectives in transcranial magnetic stimulation: Epilepsy, consciousness and the perturbational approach". Behav Neurol 2013; 27 : 155-167

McCormick D, Contreras D. "On the cellular and network bases of epileptic seizures". Annu Rev Physiol 2001; 63: 815-846

Morbidi F, Garulli A, Prattichizzo D, Rizzo C, Manganotti P, Rossi S. "Off-line removal of TMS-induced artifacts on human electroencephalography by Kalman filter". J Neurosci Methods 2007; 162: 293-302

Mutanen T, Mäki H, Ilmoniemi RJ. "The Effect of Stimulus Parameters on TMS-EEG Muscle Artifacts". Brain Stimulation 2013; 6: 371-376

Nikulin VV, Kičić D, Kähkönen S, Ilmoniemi RJ. "Modulation of electroencephalographic responses to transcranial magnetic stimulation: evidence for changes in cortical excitability related to movement". Eur J Neurosci 2003; 18: 1206-1212

Premoli I, Biondi A, Carlesso S, Rivolta D, Richardson MP. "Lamotrigine and levetiracetam exert a similar modulation of TMS-evoked EEG potentials". Epilepsia 2017; 58: 4250 
Premoli I, Castellanos N, Rivolta D, Belardinelli P, Bajo R, Zipser C et al. "TMS-EEG signatures of GABAergic neurotransmission in the human cortex". J Neurosci 2014; 34: $5603-5612$

Rogasch NC, Sullivan C, Thomson RH, Rose NS, Bailey NW, Fitzgerald PB et al. "Analysing concurrent transcranial magnetic stimulation and electroencephalographic data: A review and introduction to the open-source TESA software". Neuroimage 2017; 147: 934-951

Rogasch NC, Thomson RH, Daskalakis ZJ, Fitzgerald PB. "Short-Latency Artifacts Associated with Concurrent TMS-EEG". Brain Stimulation 2013;

Rogasch NC, Thomson RH, Farzan F, Fitzgibbon BM, Bailey NW, Hernandez-Pavon JC et al. "Removing artefacts from TMS-EEG recordings using independent component analysis: importance for assessing prefrontal and motor cortex network properties". Neuroimage 2014; 101: 425-439

Rosanova M, Casali A, Bellina V, Resta F, Mariotti M, Massimini M. "Natural frequencies of human corticothalamic circuits". J Neurosci 2009; 29: 7679-7685

Schmidt S, Bathe-Peters R, Fleischmann R, Ronnefarth M, Scholz M, Brandt SA. "Nonphysiological factors in navigated TMS studies; confounding covariates and valid intracortical estimates". Hum Brain Mapp 2015; 36: 40-49

Valentin A, Arunachalam R, Mesquita-Rodrigues A, Garcia Seoane JJ, Richardson MP, Mills KR, Alarcon G. "Late EEG responses triggered by transcranial magnetic stimulation (TMS) in the evaluation of focal epilepsy". Epilepsia 2008; 49: 470-480

Wu W, Keller CJ, Rogasch NC, Longwell P, Shpigel E, Rolle CE, Etkin A. "ARTIST: A fully automated artifact rejection algorithm for single-pulse TMS-EEG data". Hum Brain Mapp 2018; 39: 1607-1625

Ziemann U, Reis J, Schwenkreis P, Rosanova M, Strafella A, Badawy R, Muller-Dahlhaus F. "TMS and drugs revisited 2014". Clin Neurophysiol 2015; 126: 1847-1868 
Summary 
Epilepsy, resulting from an imbalance in excitation and inhibition of brain activity, is characterized by the occurrence of seizures. A patient is diagnosed with epilepsy when there have been two or more seizures, when there is evidence for a specific epilepsy syndrome, or when there has been one seizure with an increased risk of more seizures. An increased risk exists when the electroencephalogram (EEG) shows epileptiform discharges, or when structural brain abnormalities are seen on a magnetic resonance imaging (MRI) scan. In patients presenting with a single seizure without evidence for an increased risk of more seizures, uncertainty remains. Currently the only option is waiting if a second seizure occurs before there is certainty on the epilepsy diagnosis. The first line of treatment in epilepsy is starting anti-epileptic drugs (AEDs) to prevent additional seizures. Treatment efficacy is determined by seizure recurrence. Searching for an effective dosage of a certain AED (or combination of AEDs), or concluding that a patient does not respond to medication, can take up to several months.

Two major challenges in epilepsy management are to improve the diagnostic process in patients presenting with a single seizure, and to shorten the time needed to evaluate the success of AEDs in epilepsy patients. At present we mainly rely on seizure recurrence, but since seizures are disturbing events with possible damaging effects, there is a need for a new biomarker to assess the disease status.

With Transcranial Magnetic Stimulation (TMS) short magnetic pulses are applied to the cortex. The strength of the response of the activated neurons to this stimulus can be used as a measure for the balance in excitation and inhibition of brain activity. TMS may be a useful new tool to study (changes in) this balance, and is therefore of interest in the field of epilepsy. In this thesis we focus on the TMS evoked potential (TEP): the EEG response induced by TMS obtained after averaging over multiple single TMS pulses. As epilepsy is associated with a higher excitability, the TEP may be different in epilepsy patients. If so, the TEP could serve as a potential biomarker for epilepsy diagnostics, and as a monitoring tool for evaluating treatment efficacy.

Two large amplitude artifacts hinder the evaluation of the TEP. The short-lasting TMS pulse artifact gives rise to filtering problems, whereas the long-lasting muscle activation artifact overlies the first TEP components. Principal Component Analysis (PCA) applied on single TMS-EEG trials results in a distribution of the large amplitude artifacts in the first principal components and the relatively small 
amplitude neuronal activity in later principal components. After consecutive removal of the principal components from the TMS-EEG data, the subsequent reduction in artifact amplitude as well as in TEP amplitude can be evaluated. Using PCA both artifacts are effectively reduced, thereby revealing the first TEP components and allowing further TEP analysis (chapter 2).

TMS pulses are accompanied by a clicking sound, inducing an auditory evoked potential (AEP) which is superimposed to the TEP. This AEP can be minimized by using a headphone playing noise and with a layer of foam between the coil and head (chapter 3). Applying TMS-EEG to a completely deaf person proves that the TEP is definitely not only evoked by sound alone. However, the contribution of the somatosensory evoked potential (SSEP) to the TEP still needs to be elucidated.

For a clinical application of TMS-EEG, the variation of the TEP is of great importance. It appears that the variation between subjects is large, which may make it difficult to differentiate healthy from disease. The within subject variation of the TEP seems to be smaller, possibly enabling follow-up measurements for therapy evaluation. The motor threshold and motor evoked potential do not vary significantly during the day, and the TEP is highly reproducible at different TMS-EEG sessions during daytime (chapter 4).

A study in a group of 14 epilepsy patients showed a higher motor threshold and a larger amplitude of the TEP when compared to healthy controls (chapter 5). Part of these differences may possibly be explained by the use of AEDs. Therefore, future research to assess the diagnostic utility of the TEP should focus on performing TMSEEG studies in first-seizure patients as well as in drug naive epilepsy patients. In addition, TMS-EEG should be applied before and after taking AEDs to evaluate the utility of the TEP in individual follow-up measurements. 


\section{Samenvatting}


Epilepsie, veroorzaakt door een disbalans tussen activatie en remming van hersenactiviteit, wordt gekenmerkt door het optreden van insulten. Een patiënt wordt gediagnosticeerd met epilepsie als er twee of meer insulten zijn geweest, als er aanwijzingen zijn voor een specifiek epilepsie syndroom, of als er één insult is geweest met een verhoogde kans op meer insulten. Een verhoogde kans op insulten is aanwezig als het electroencefalogram (EEG, hersenfilmpje) epileptiforme afwijkingen laat zien, of als er structurele hersenafwijkingen te zien zijn op een MRI scan. In patiënten die slechts één insult hebben doorgemaakt, en waarbij er geen verhoogd risico op meer insulten wordt gevonden, is de verwachting onzeker. Momenteel is de enige optie om af te wachten of er een tweede insult plaatsvindt voordat er zekerheid is over de diagnose epilepsie. De normale behandeling bij epilepsie bestaat uit het starten met anti-epileptica (AEDs) om verdere insulten te voorkomen. De effectiviteit van deze behandeling wordt bepaald door het wel of niet opnieuw optreden van insulten. Het zoeken naar een effectieve dosering van een AED (of combinatie van AEDs), of het concluderen dat een patiënt niet voldoende reageert op medicatie, kan meerdere maanden in beslag nemen.

Twee belangrijke uitdagingen bij epilepsie zijn om de diagnostiek te verbeteren in patiënten bij wie slechts één insult heeft opgetreden, en om de tijd te verkorten die nodig is om het succes van de behandeling met AEDs te evalueren. We vertrouwen hierbij nu vooral op de herhaling van insulten, maar omdat insulten heftig zijn om door te maken en ook mogelijk schadelijke effecten hebben, is er een behoefte aan een nieuwe biomarker om de ziekte te beoordelen.

Met Transcraniële Magnetische Stimulatie (TMS) worden korte magnetische pulsen op de hersenschors gegeven. De sterkte van de reactie van de geactiveerde hersencellen op deze stimulus kan gebruikt worden als een maat voor de balans tussen activatie en remming. TMS zou een bruikbare nieuwe tool kunnen zijn om (veranderingen in) deze balans te bestuderen, en is daarom interessant bij epilepsie. In deze thesis leggen we de focus op de TMS evoked potential (TEP): de EEG reactie na TMS die wordt verkregen na het middelen over meerdere TMS pulsen. Omdat epilepsie geassocieerd is met een hogere prikkelbaarheid, zou de TEP anders kunnen zijn bij epilepsie patiënten. Als dat zo is, dan zou de TEP een mogelijke biomarker kunnen zijn voor epilepsie diagnostiek, en als een monitoring tool om het succes van de behandeling te evalueren. 
Twee artefacten met een grote amplitude maken de beoordeling van de TEP lastig. Het kortdurende TMS puls artefact geeft filterproblemen, en het langdurende spier activatie artefact ligt over de eerste componenten van de TEP heen. Bij Principiële Component Analyse (PCA), uitgevoerd op individuele TMS-EEG signalen, wordt een verdeling gevonden van de artefacten met een grote amplitude in de eerste principiële componenten en de activiteit van hersencellen met een lage amplitude in latere principiële componenten. Na het één voor één verwijderen van de principiële componenten van de TMS-EEG data kan de afname in artefact amplitude en TEP amplitude beoordeeld worden. Met PCA kunnen beide artefacten verminderd worden, waarbij de eerste TEP componenten zichtbaar worden en verdere TEP analyse mogelijk is (hoofdstuk 2).

Het geven van TMS pulsen gaat gepaard met een klikkend geluid, waarmee een auditore evoked potential (AEP) wordt veroorzaakt die boven op de TEP ligt. Deze AEP kan verminderd worden door een koptelefoon te gebruiken waardoor ruis wordt afgespeeld en met een laag schuim tussen de spoel en het hoofd (hoofdstuk 3). Toepassing van TMS-EEG bij een doof persoon bewijst dat de TEP zeker niet alleen door geluid wordt veroorzaakt. De contributie van de somatosensorische evoked potential (SSEP) moet echter nog worden uitgezocht.

De variatie van de TEP is erg belangrijk voor een mogelijke klinische applicatie van TMS-EEG. Het lijkt erop dat de variatie tussen proefpersonen groot is, waardoor het onderscheiden van gezond en ziek lastig zou kunnen worden. De variatie binnen een proefpersoon lijkt kleiner te zijn, dus vervolgmetingen voor het evalueren van de behandeling zijn wellicht wel mogelijk. De motor drempel en motor evoked potential variëren niet gedurende de dag, en de TEP is erg reproduceerbaar tussen verschillende TMS-EEG sessies gedurende de dag (hoofdstuk 4).

Een studie in veertien epilepsie patiënten liet zien dat ze in vergelijking met gezonden een hogere motor drempel en een hogere amplitude van de TEP hadden (hoofdstuk 5). Een deel van deze verschillen zou wellicht verklaard kunnen worden door het gebruik van AEDs. Daarom moet toekomstig onderzoek naar de mogelijke diagnostische toepassing van de TEP zich focussen op het uitvoeren van TMS-EEG studies in patiënten met een eerste insult en in patiënten met epilepsie die geen AEDs gebruiken. Daarnaast zou TMS-EEG toegepast moeten worden voor en na het starten van AEDs om te bekijken of de TEP bruikbaar is voor individuele vervolgmetingen. 


\section{Dankwoord}


Het heeft even geduurd, maar eindelijk schrijf ik dan mijn dankwoord. Zonder twijfel het meest gelezen onderdeel van een proefschrift. Ik wil graag de mensen noemen die hebben bijgedragen aan dit onderzoek en me hebben gesteund de afgelopen jaren.

Michel, bedankt dat je mij de kans hebt gegeven om bij jou te komen promoveren in de vakgroep Clinical Neurophysiology. Je enthousiasme over de neurologie en de wetenschap heeft me altijd erg geïnspireerd. Ik kreeg bij jou de vrijheid en ruimte om mezelf in diverse aspecten van onderzoek en onderwijs te ontwikkelen, wat mij betreft essentieel bij het opleiden van een zelfstandig wetenschapper. Je hebt me steeds gemotiveerd om het promotietraject alsnog af te ronden, en gaf me altijd het gevoel dat dit binnen handbereik lag. Uiteindelijk had je (uiteraard) gelijk, en ben ik heel blij dat ik nu mijn proefschrift mag verdedigen. Bedankt!

Alle proefpersonen en epilepsie patiënten: ik waardeer het heel erg dat jullie mee wilden doen met mijn onderzoek. Dankzij jullie zijn de resultaten van dit proefschrift tot stand gekomen. Bedankt voor jullie vrijwillige deelname.

Prof. Vonck, prof. Stegeman, dr. Schelhaas, prof. van Gils en dr. van Asseldonk, bedankt voor het plaatsnemen in de promotiecommissie.

Ik wil graag de neurologen van het Medisch Spectrum Twente bedanken voor de medewerking bij het includeren van de epilepsie patiënten. Dr. Bezooijen van de radiologie heeft gedurende twee avonden zelf de MRI scans van de gezonde proefpersonen gemaakt, en prof. dr. Van der Palen heeft meerdere malen advies gegeven over de METC protocollen en statistische analyses. Dank daarvoor.

De KNF van het MST was een fijne plek om te werken, tijdens mijn stage, afstuderen en promotie. Bedankt voor de leuke gesprekken in de koffiekamer en uitgebreide uitleg bij de KNF onderzoeken. In het bijzonder wil ik Carin noemen. Ik ben heel blij dat er zo'n enthousiaste KNF laborant bij het onderzoek betrokken was. Dankzij jou zijn veel patiënten geïncludeerd en gemeten, je haalde ze zelfs persoonlijk bij huis op. Je weet altijd wat te doen bij problemen, en niets is je te veel. Dankjewel.

De meeste tijd heb ik doorgebracht bij CNPH. Wat was het gezellig met alle onderzoekers: Sid, Chin, Shaun, Bas-Jan, Floor, Cecile, Jessica, Marleen, Annika, Barry. Ik heb het echt onwijs naar m'n zin gehad met jullie. Een selectie: de UT 
sportdag, de bbq bij Michel in de achtertuin, de fantastische trips naar Rome en San Diego, Sinterklaas met Michel als goedheiligman, vele etentjes, en alle andere activiteiten en feestjes. Met Marleen, Floor, Cecile en Jessica deelde ik vier jaar lang lief en leed in ons kippenhok. Ook wil ik de toenmalige studenten Benjamin, Mark, Inês en Anne-Wil die ik mocht begeleiden, en die in meer of mindere mate hebben bijgedragen aan mijn publicaties, bedanken. Ik wens jullie allemaal het allerbeste!

Tanja, jij bent zoveel meer dan een secretaresse voor mij geweest. Ik wil je ontzettend bedanken voor je begrip, oprechte interesse en positiviteit. Je kantoor verliet ik altijd weer vol goede moed en meermaals met een last van mijn schouders.

Ik wil alle oud-collega's van TG en het ECTM bedanken voor de goede tijd die ik bij jullie op de gang heb gehad, met veel leuke gesprekken en natuurlijk drie-uurkoffietijd. Wim bedankt nog voor het fixeren van de TMS spoel. Mijn oudkamergenoten Selma (en de plant) en Marleen (review?) wil ik bedanken voor de plezierige tijd samen. Esther, het was fijn om met jou te mogen werken aan het TG netwerk.

Ik ben heel blij dat ik bij Demcon een leuke en uitdagende baan heb gevonden. Ik wil al mijn collega's bedanken voor de prettige sfeer en goede samenwerking. Michiel, heel erg bedankt voor het mede faciliteren van het laatste stukje van mijn promotie. Mijn kamergenoten bedank ik voor het meeleven met de (soms stressvolle) laatste loodjes. Patricia, bedankt voor al je adviezen bij het drukken van de boekjes.

Marleen, we hebben samen onze promotie projecten doorlopen en al die tijd een kamer gedeeld. Ik heb superveel aan je gehad, zowel inhoudelijk als op persoonlijk vlak. We werkten zo goed samen dat we al die jaren al tegen elkaar zeiden: we moeten echt op zoek naar een duobaan. Ik ben blij dat je een hele mooie baan in het MST hebt gevonden, en dat je vandaag naast me staat als paranimf.

Annika, mijn andere paranimf, wat ben ik blij met jou als mede TMS-er. Je begon nadat ik al weg was, maar we hebben nog veel samengewerkt aan een paar mooie publicaties. Het was fijn om alle ins en outs van TMS onderzoek te kunnen delen. Naast de inhoudelijke samenwerking was je ook een grote steun en motivator de afgelopen tijd, dank daarvoor. Heel veel succes met het afronden van jouw promotie! 
Anne en Joyce, jullie ken ik allebei al vanaf de middelbare school. Anne, wat was het bijzonder om tegelijkertijd voor het eerst moeder te worden en wat hebben we veel geappt over slaapjes/hapjes/huiltjes en daarnaast nog zoveel meer. Joyce, al die jaren samen met de bus naar de UT, heel fijn en vertrouwd om met een nuchtere (bijna-) streekgenoot de opleiding te doen. Met jullie allebei heb ik de hoogte- en dieptepunten van het promoveren en allerlei carrière overwegingen kunnen delen. Ook al zien we elkaar een stuk minder vaak, zeker nu we een druk gezinsleven hebben, het is altijd weer gezellig en vertrouwd. Dat laatste geldt ook voor al mijn andere goede vrienden, die met enige regelmaat voor de broodnodige afleiding hebben gezorgd (koffie/eetclub/festivals/vakanties/enz). Bedankt voor de steun en lieve woorden de afgelopen jaren.

Lieve papa, bedankt voor je steun en dat je altijd voor me klaarstaat. Ik heb heel veel respect voor jou en hoe je in het leven staat. Mama, wat is het ontzettend verdrietig dat je niet meer bij ons bent, ik mis je nog elke dag. Maar ik weet zeker dat je meekijkt en geniet van al dit spektakel. Lieve broers en zus (en schoonzussen en zwager), wat hebben we het goed samen. Onze hechte band betekent veel voor me, het voelt altijd als thuiskomen bij elkaar. Bedankt ook iedereen voor de ontelbare keren 'wanneer ga je nou afstuderen?' (-). Ook mijn schoonfamilie wil ik bedanken voor de gezelligheid en interesse.

Arnout, mijn jeugdliefde, veilige haven en mede-slechte-grappenmaker. Wat hebben we al veel meegemaakt samen, hele mooie tijden, maar ook mindere tijden. Ik zou het met niemand anders willen delen. Wat is nou de kans dat je zo iemand tegenkomt in je leven? Ik zeg 50\%.

Mijn kinderen, Guus en Stella, ik geniet met volle teugen van jullie. Lieve Guus, mijn eigenwijze knuffelkont, wat gaat de tijd snel! En wat is het leuk om de wereld opnieuw te zien door jouw ogen. Lieve kleine Stella, hoe bizar is het dat we door jou zelf te maken kregen met het onderwerp van mijn promotie. Gelukkig gaat het weer goed met je en ben je inmiddels een vrolijke, levenslustige (en ondeugende...) dreumes. Ik hou zielsveel van jullie twee. 
About the author 
Esther ter Braack (June $4^{\text {th }} 1984$, Enschede) attended Het Assink in Haaksbergen, from which she obtained her VWO degree in 2002. She then started her study in Biomedical Engineering, but after one year she switched to Technical Medicine at the University of Twente, Enschede. After the 3-year bachelor program she continued with the master track Medical Signaling. During this master program she performed internships at the departments of intensive care medicine and clinical neurophysiology at the Medisch Spectrum Twente in Enschede, at the department of cardiology at UMC St Radboud Hospital in Nijmegen, and at the department of pediatric urology at UMC Utrecht. In August 2009 she obtained her master degree at the thoracic intensive care unit under the supervision of prof. dr. Jan Grandjean, dr. Wytze Vermeijden and prof. dr. ir. Michel van Putten, with a thesis on electrical muscle stimulation in intensive care patients to prevent muscle weakness.

In 2009 Esther started her $\mathrm{PhD}$ project at the department of Clinical Neurophysiology of the University of Twente under the supervision of prof. dr. ir. Michel van Putten. This thesis describes the results of that project on combining transcranial magnetic stimulation and electroencephalography in healthy subjects and epilepsy patients.

From 2014 to 2016 Esther worked at the Technical Medicine department at the University of Twente, coordinating student internships and collaborations between the university chairs and hospital departments in the Netherlands. Since 2016 she works at DEMCON Advanced Mechatronics, Enschede, where she coordinates and performs clinical evaluation studies for the medical devices that are developed at the company.

Esther is married to Arnout Spanjer and together they have a son, Guus (2014), and a daughter, Stella (2016). 


\section{List of publications}




\section{Journal publications}

ter Braack EM, de Jonge B, van Putten MJ. 'Reduction of TMS Induced Artifacts in EEG Using Principal Component Analysis'. IEEE Transactions on Neural Systems and Rehabilitation Engineering 2013;21(3):376-382

ter Braack EM, de Vos CC, van Putten MJ. 'Masking the auditory evoked potential in TMS-EEG: a comparison of various methods'. Brain Topography 2015;28:520528

ter Braack EM, Koopman AE, van Putten MJ. 'Early TMS evoked potentials in epilepsy: a pilot study'. Clinical Neurophysiology 2016;127:3025-3032

de Goede AA, ter Braack EM, van Putten MJ. 'Single and paired pulse transcranial magnetic stimulation in drug naïve epilepsy'. Clinical Neurophysiology 2016;127:3140-3155

Bauer PR, de Goede AA, ter Braack EM, van Putten MJ, Gill RD, Sander JW. 'Transcranial magnetic stimulation as a biomarker for epilepsy'. Brain 2017;140(3):e18

ter Braack EM, de Goede AA, van Putten MJ. 'The motor threshold and TMS-EEG evoked potentials are constant during daytime '. Submitted in revised form (Brain Topography)

de Goede AA, ter Braack EM, van Putten MJ. 'Varying the coil location and orientation in single and paired pulse TMS'. Submitted in revised form (Brain Topography) 


\section{Conference contributions}

ter Braack EM, de Jonge B, de Vos CC, van Putten MJ. 'Masking the auditory evoked potential in TMS-EEG'. 14th European Congress of Clinical Neurophysiology, June 2011, Rome

ter Braack EM, Silva Santos I, CJ Eertman, van Putten MJ. 'TMS-EEG: A new diagnostic tool in epilepsy?'. Update@ Kempenhaeghe, March 2012, Heeze

ter Braack EM, Silva Santos I, CJ Eertman, van Putten MJ. 'Abnormal TMS/EEG responses in Epilepsy'. 2012 Annual Meeting of the American Epilepsy Society, December 2012, San Diego

ter Braack EM, Silva Santos I, CJ Eertman, van Putten MJ. 'Abnormal TMS/EEG responses in Epilepsy'. 5th International conference on Non-invasive Brain Stimulation, March 2013, Leipzig

ter Braack EM, de Goede AA, van Putten MJ. 'Variation during the day of the TMSEEG evoked potential'. 5th International conference on Transcranial Brain Stimulation, September 2016, Göttingen

\section{Oral presentations}

'TMS and EEG', ANT Neuromeeting 2011, Beaune, France.

'Transcraniële Magnetische Stimulatie', Lustrumcongres voor Klinische Fysica 2013, de Lutte 
